University of Tennessee Health Science Center

UTHSC Digital Commons

\title{
Investigation of Enhancement of Furosemide Solubilization with Cyclodextrins and a Novel Octenyl Succinate Anhydride Starch
}

\author{
Rui Zhu \\ University of Tennessee Health Science Center
}

Follow this and additional works at: https://dc.uthsc.edu/dissertations

Part of the Medicinal and Pharmaceutical Chemistry Commons, and the Pharmaceutics and Drug Design Commons

\section{Recommended Citation}

Zhu, Rui , "Investigation of Enhancement of Furosemide Solubilization with Cyclodextrins and a Novel Octenyl Succinate Anhydride Starch" (2012). Theses and Dissertations (ETD). Paper 323. http://dx.doi.org/10.21007/etd.cghs.2012.0385. 


\title{
Investigation of Enhancement of Furosemide Solubilization with Cyclodextrins and a Novel Octenyl Succinate Anhydride Starch
}

\begin{abstract}
Solubility behavior is one of the most challenging aspects for drug commercialization and often the main reason of drug that do not reach to its full potential. Now nearly $60 \%$ new chemical entities possess solubility problems, whereas practically no drug products with less than $10 \mu \mathrm{g} / \mathrm{ml}$ solubility in 70's or 80 's. There is an ever increasing need to develop new formulation techniques and exicipients with novel mechanisms of action. Various techniques have been applied to enhance the drug solubility such as cosolvents, particle size reduction, lipid based drug delivery systems, nanosuspension, use of surfactants, salt formation, cyclodextrin complexes and solid dispersion etc.
\end{abstract}

Chapter 1 is an introduction into biopharmaceutics classification system (BCS) and novel drug solubilization techniques. It provides a brief history of the BCS and its application in drug development. It also looks into the recent poorly water soluble drugs approved by the Food and Drug Administration and the formulation techniques that these drugs used.

Chapter 2 is a brief history of solid dispersion, including its theoretical basis, preparation methods, and characterization and quality control tools. Several drug products using solid dispersion technique are also reviewed. This chapter is intended to combine recent literature on solid dispersion technology for solubility enhancement with various preparation methods, evaluation parameters and commonly used polymer carriers.

Chapter 3 explores the applicabability of a cold water soluble modified starch, octenyl succinic anhydrate starch (OSA starch) as solid dispersion carrier to increase the aqueous solubility and dissolution rate of poorly water soluble drugs. When using OSA starch in solid dispersions via spray drying techniques, the hypothesis is that drug is dissolved in the starch solution, when water evaporate via spray drying, drug will be solidified together with starch and mostly the drug is in amorphous form because the rapid spray drying process (seconds) prevents drug crystallization. In this system, the starch is homogenously distributed in the matrix and thus prevents drug crystalline growth as a characteristic of the polymer. When drug is dispersed in starch solution, the starch will encapsulate drug particles to complete the microencapsulation process and micro-scale drug particles will be retained in the starch matrix. These properties will be helpful in enhancing drug solubility and dissolution rate.

Chapter 4 explores a solid dispersion of furosemide and sulfobutylether- $\beta$-cyclodextrin which was another derivative excipient of starch. Solid dispersion preparation method and mechanism are also discussed. The work serves as a positive control for OSA starch solid dispersion. Comparison and contrasting the two solid dispersions assist in the interpretation of OSA starch solid dispersions.

\author{
Document Type \\ Dissertation \\ Degree Name \\ Doctor of Philosophy (PhD) \\ Program \\ Pharmaceutical Sciences \\ Research Advisor \\ James R. Johnson, Ph.D.
}




\section{Keywords}

Hassan Almoazen, Ph.D. Gerald M. Rajan, Ph.D. George C. Wood, Ph.D. Arthur D. Straughn, Ph.D

\section{Subject Categories}

Medicinal and Pharmaceutical Chemistry | Medicine and Health Sciences | Pharmaceutics and Drug Design | Pharmacy and Pharmaceutical Sciences

\section{Comments}

Two year embargo expired August 2014 


\title{
Investigation of Enhancement of Furosemide Solubilization with Cyclodextrins and
} a Novel Octenyl Succinate Anhydride Starch

\author{
A Dissertation \\ Presented for \\ The Graduate Studies Council \\ The University of Tennessee \\ Health Science Center
}

In Partial Fulfillment

Of the Requirements for the Degree

Doctor of Philosophy

From The University of Tennessee

By

Rui Zhu

August 2012 
Copyright (C) 2012 by Rui Zhu. All rights reserved. 


\title{
DEDICATION
}

\author{
To \\ My husband, Zhao Wang \\ My children, Sophia \& Aaron \\ And \\ My parents and sister \\ For \\ Their endless love and continuous support
}




\section{ACKNOWLEDGEMENTS}

I would like to gratefully and sincerely thank Dr. James R. Johnson for his understanding, trust, patience, guidance and most importantly, his support during my graduate studies at UTHSC. His mentorship was paramount in providing an open view consistent my long career goals. He guided and encouraged me not only to grow as a formulator but also as an independent thinker. I deeply appreciated the given opportunity to develop individuality and self-sufficiency by being allowed to work in an independent environment. Dr. Johnson, I thank you, for everything you've done for me.

I would also like to thank my committee members: Drs. Almoazen, Rajan, Straughn and Wood. Their good suggestions and questions to the experiment design and research rationale helped me to solve the problems and inspired new ideas for my project continuity.

I want to give my special thanks and most appreciation to Dr. Atul Shukla. His generous, understanding and tolerant to accept me as his and Dr. Johnson's student lighten the road of my life. He gave his hand to help me out of difficulties and then have the opportunity to continue my study at UTHSC.

My sincere appreciation was also extended to Mr. Ed Brunson, Dr. Carmen Popescu, Michael Moore, Jean-Yves PIERQUIN for their help in my project; Dr. Wei Li for his great help in FTIR spectrum interpretation for solid dispersion mechanism.

I acknowledge the support of the following companys: Surface Measurement Systems (London, UK), Woodbridge Scientific LLC, Micromeritics, Carver press to provide dynamic vapor sorption instrument, dissolution instrument and instruments measuring particle size distribution, surface area, true density, manual tablet press, etc.

I also would like to thank my colleagues and lab-mates, Drs. Wen Qu, Paras Jain; Yan lv and Sonia, Suresh, Yinqi, Jin, Frank and Hao for their help and support over these years.

I would also like to extend my deepest gratitude to my family. Thanks to my parents: Guangyuan, Zhu and Yumei, Chen for their long years love and encouragement; my sister, Linlin Zhu, for everything she's done for the family to let me concentrate on my studies. I also give my sincere thanks to my parents-in-law, for their great and unselfish help and consideration to my children. Finally, I thank my husband and my children with their support for me during my studies. 


\begin{abstract}
Solubility behavior is one of the most challenging aspects for drug commercialization and often the main reason of drug that do not reach to its full potential. Now nearly $60 \%$ new chemical entities possess solubility problems, whereas practically no drug products with less than $10 \mu \mathrm{g} / \mathrm{ml}$ solubility in 70's or 80 's. There is an ever increasing need to develop new formulation techniques and exicipients with novel mechanisms of action. Various techniques have been applied to enhance the drug solubility such as co-solvents, particle size reduction, lipid based drug delivery systems, nanosuspension, use of surfactants, salt formation, cyclodextrin complexes and solid dispersion etc.
\end{abstract}

Chapter 1 is an introduction into biopharmaceutics classification system (BCS) and novel drug solubilization techniques. It provides a brief history of the BCS and its application in drug development. It also looks into the recent poorly water soluble drugs approved by the Food and Drug Administration and the formulation techniques that these drugs used.

Chapter 2 is a brief history of solid dispersion, including its theoretical basis, preparation methods, and characterization and quality control tools. Several drug products using solid dispersion technique are also reviewed. This chapter is intended to combine recent literature on solid dispersion technology for solubility enhancement with various preparation methods, evaluation parameters and commonly used polymer carriers.

Chapter 3 explores the applicabability of a cold water soluble modified starch, octenyl succinic anhydrate starch (OSA starch) as solid dispersion carrier to increase the aqueous solubility and dissolution rate of poorly water soluble drugs. When using OSA starch in solid dispersions via spray drying techniques, the hypothesis is that drug is dissolved in the starch solution, when water evaporate via spray drying, drug will be solidified together with starch and mostly the drug is in amorphous form because the rapid spray drying process (seconds) prevents drug crystallization. In this system, the starch is homogenously distributed in the matrix and thus prevents drug crystalline growth as a characteristic of the polymer. When drug is dispersed in starch solution, the starch will encapsulate drug particles to complete the microencapsulation process and micro-scale drug particles will be retained in the starch matrix. These properties will be helpful in enhancing drug solubility and dissolution rate.

Chapter 4 explores a solid dispersion of furosemide and sulfobutylether- $\beta$-cyclodextrin which was another derivative excipient of starch. Solid dispersion preparation method and mechanism are also discussed. The work serves as a positive control for OSA starch solid dispersion. Comparison and contrasting the two solid dispersions assist in the interpretation of OSA starch solid dispersions. 


\section{TABLE OF CONTENTS}

CHAPTER 1. BACKGROUND.....................................................................................

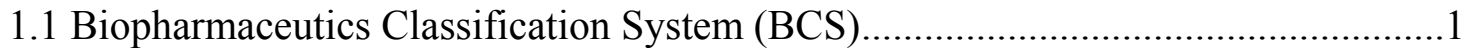

1.1.1 Relation between Do, Dn, An, and dissolution.................................................

1.1.2 Determination of solubility .........................................................................

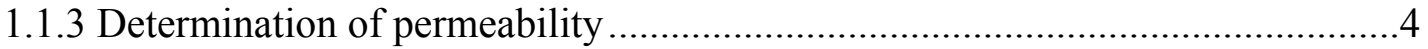

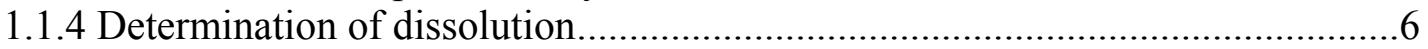

1.2 Formulation Strategies for Poorly Water Soluble Drug Development.......................8

1.2.1 Nanotechnology in poorly water soluble drug development ..............................

1.2.1.1 Formulation rationale and ingredient function ........................................ 9

1.2.1.2 Nanosuspension preparation methods ..................................................... 10

1.2.1.3 Characterization and key factors affecting product quality ..................... 13

1.2.1.4 Commercial products and promising drug product status ....................... 13

1.2.2 Self-emusifying drug delivery system ..........................................................13

1.2.2.1 Basic principle and excipient function................................................... 13

1.2.2.2 Phase diagram and characterization tools ............................................. 14

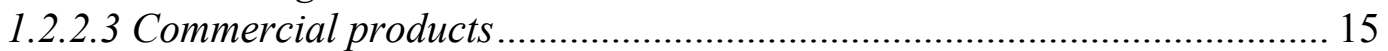

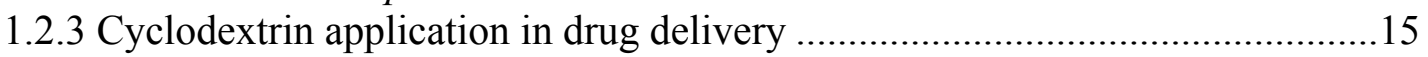

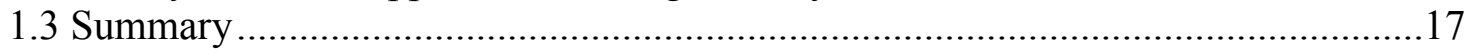

CHAPTER 2. SOLID DISPERSION REVIEW ............................................................20

2.1 Definition and History of Solid Dispersion ........................................................20

2.2 Dissolution Rate or Solubility Enhancement Mechanism from Solid Dispersion...21

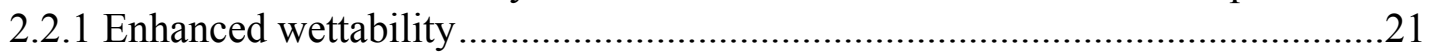

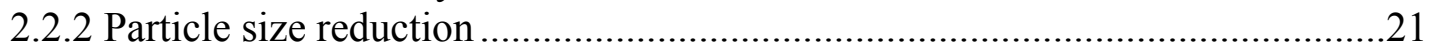

2.2.3 Reduced aggregation and agglomeration ......................................................22

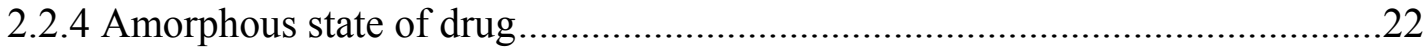

2.3 Preparation Methods ……………..................................................................22

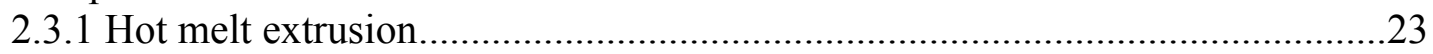

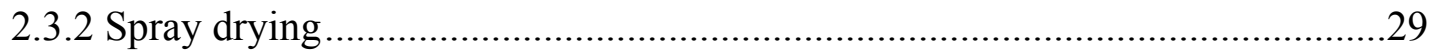

2.3.3 Supercritical fluid extrusion......................................................................

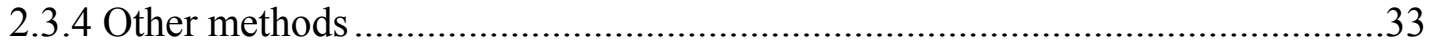

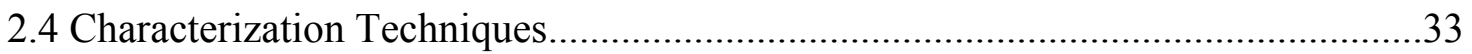

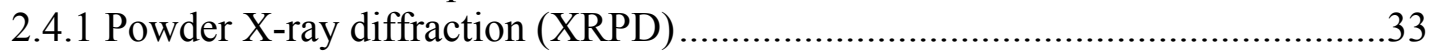

2.4.2 Thermal analysis methods.............................................................................

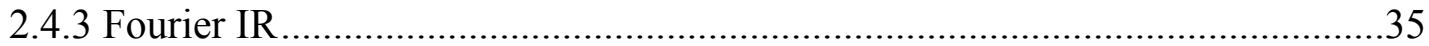

2.4.4 Scanning differential microscopy (SEM) .......................................................

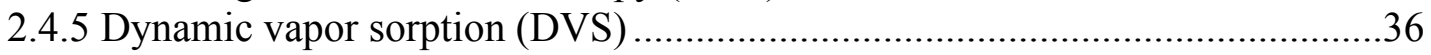

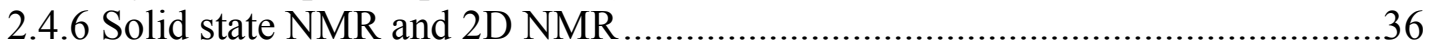

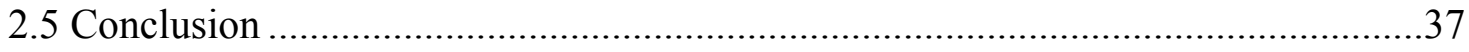




\section{CHAPTER 3. AQUEOUS SOLUBILITY, DISSOLUTION, PHYSICOCHEMICAL CHARACTERIZATION AND PHYSICAL STABILITY OF SOLID DISPERSIONS OF FUROSEMIDE AND OCTENYL SUCCINIC ANHYDRATE STARCH ............................................38}

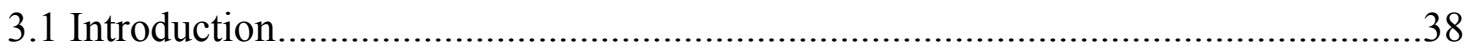

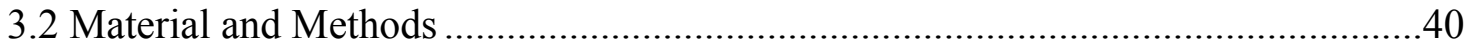

3.2.1 Material ............................................................................................40

3.2.2 Preparation of furosemide-loaded solid dispersions ..........................................40

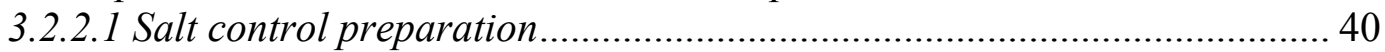

3.2.2.2 Solution solid dispersion preparation (Soln. SD) ..................................... 41

3.2.2.3 Suspension solid dispersion preparation (Susp. SD) .................................. 41

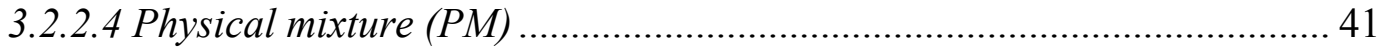

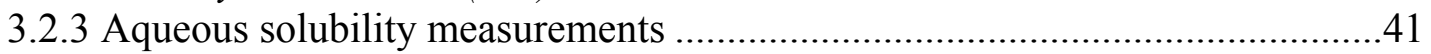

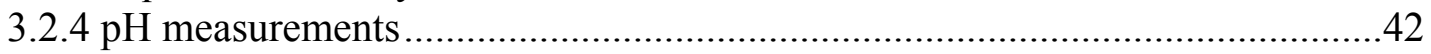

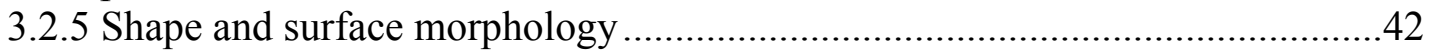

3.2.6 Crystallinity, thermal and spectroscopic properties........................................42

3.2.7 Humidity effect on solid dispersion and Dynamic Vapor Sorption (DVS) ......42

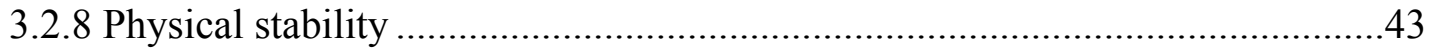

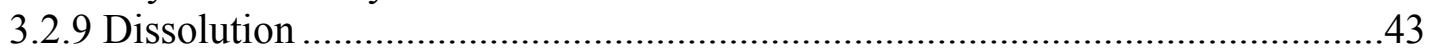

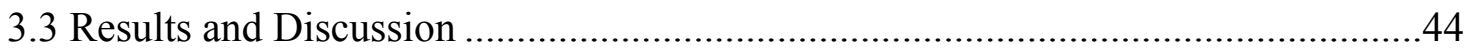

3.3.1 Aqueous solubility and $1 \%$ dispersion $\mathrm{pH}$..................................................4

3.3.2 Shape and surface morphology ....................................................................4

3.3.3 Crystallinity (XRPD) and spectroscopic properties (FTIR) ………………....47

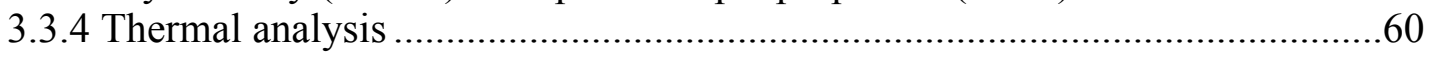

3.3.5 Humidity induced crystallization and DVS ………....................................62

3.3.6 Physical stability test (XRPD) .................................................................66

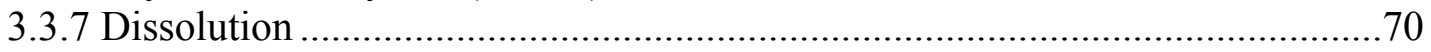

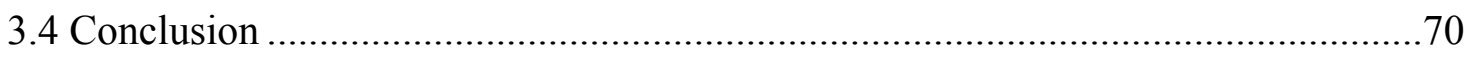

\section{CHAPTER 4. PHASE SOLUBILITY OF FUROSEMIDE WITH HP-B-CD AND SBE-B-CD, PREPARATION AND CHARACTERIZATION OF SOLID DISPERSIONS OF FUROSEMIDE AND SBE-B-CD .........................................74}

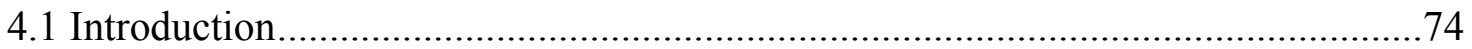

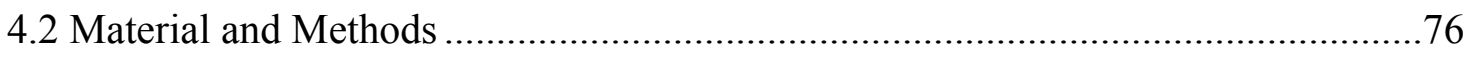

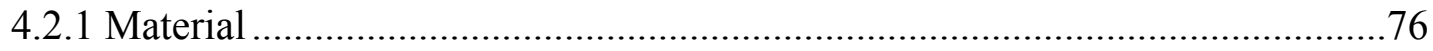

4.2.2 Phase solubility, apparent stability constant, complex efficiency …..................77

4.2.3 Preparation of furosemide: SBE- $\beta$-CD solid dispersion ....................................77

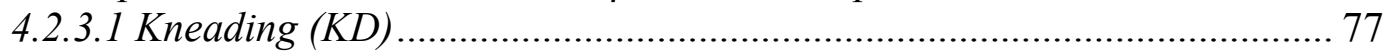

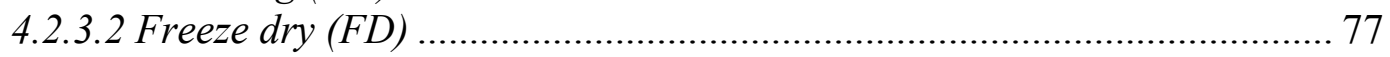

4.2.3.3 Spray drying $(S D)$ and physical mixture ............................................... 78

4.2.4 Aqueous solubility of solid dispersion in water............................................78

4.2.5 Solid dispersion powder characterization ...................................................78

4.2.6 Shape and surface morphology .................................................................78

4.2.7 Drug content in solid dispersion powder ........................................................79

4.2.8 Spectroscopic and thermal characteristics ……….......................................79

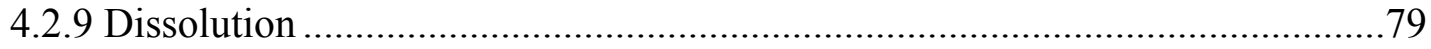

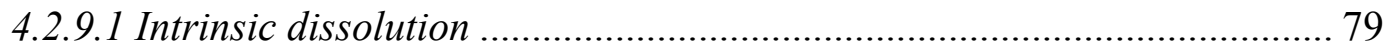




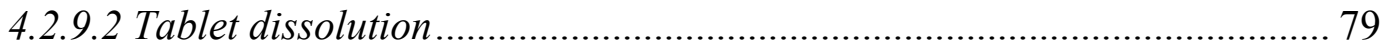

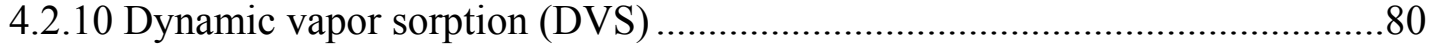

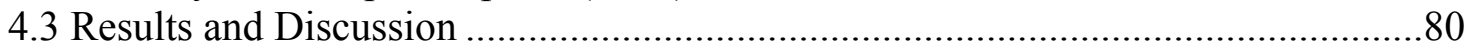

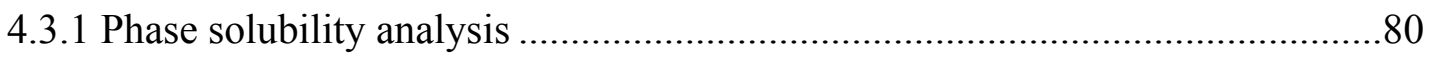

4.3.2 Aqueous solubility of solid dispersion in water........................................ 80

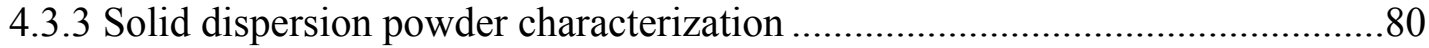

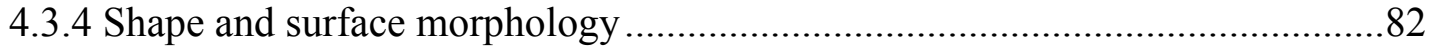

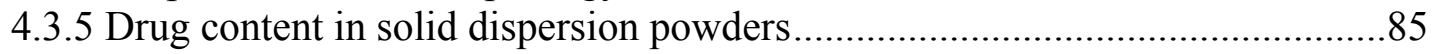

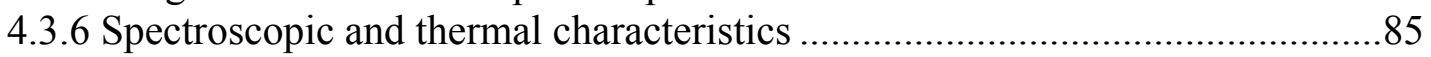

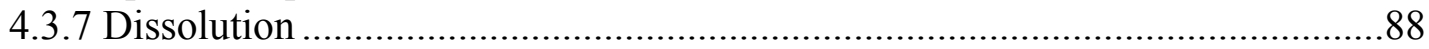

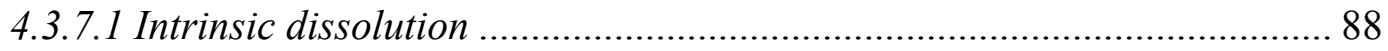

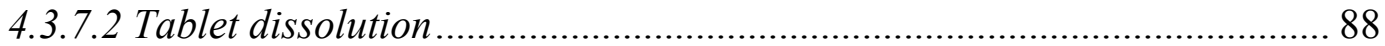

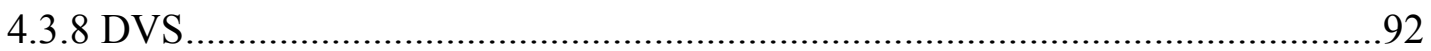

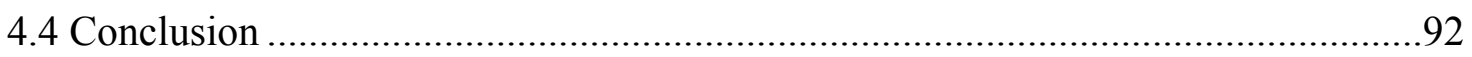

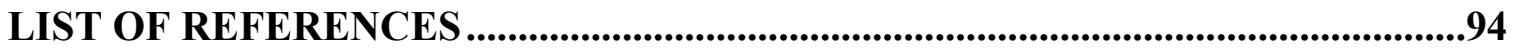

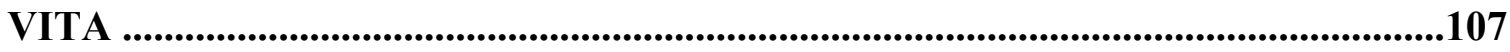




\section{LIST OF TABLES}

Table 1-1. Summary of commercial SEDDS drug products....................................16

Table 1-2. Summary of drug products using cyclodextrin......................................19

Table 2-1. Examples of commercial solid dispersions...........................................25

Table 3-1. Ingredients of two tablet formulations................................................43

Table 3-2. Summary of aqueous solubility and $\mathrm{pH}$ of $1 \%$ dispersion $(\mathrm{w} / \mathrm{v}) \ldots \ldots \ldots \ldots \ldots . . . . .46$

Table 3-3. Tg and heat capacity (Cp) of control I (before and after treated with

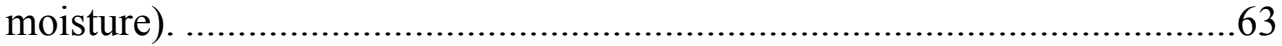

Table 3-4. $\quad \mathrm{pH}$ measurements of control I before and after water vapor sorp-desorp

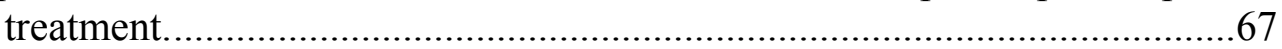

Table 3-5. Comparison of \%drug release in two formulations at $60 \mathrm{~min}$ point in SGF (pH 1.2).

Table 4-1. K 1:1 and CE of phase-solubility studies of furosemide with SBE- $\beta$-CD and $\mathrm{HP}-\beta-\mathrm{CD}$.

Table 4-2. Aqueous solubility of furosemide-CDs complex at $25^{\circ} \mathrm{C}$.

Table 4-3. Solubility of furosemide in solid dispersion prepared with three methods and the ratios of total solubility to the intrinsic solubility $(n=3)$.

Table 4-4. Particle size, specific surface area, true density and bulk density of solid

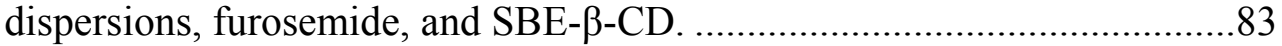

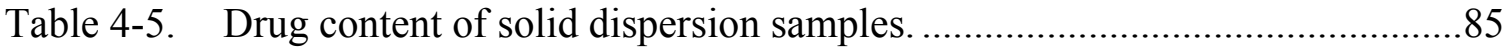




\section{LIST OF FIGURES}

Figure 1-1. Correlation between human permeability and fraction dose absorbed..........2

Figure 1-2. Relationship between Dn, Do and An.................................................4

Figure 1-3. Caco-2 cell permeability model diagram...............................................5

Figure 1-4. Intestinal perfusion model...........................................................6

Figure 1-5. Function of polymer and surfactant in nanoparticles................................10

Figure 1-6. Relation in nanoparticles, polymer, surfactant and water molecules............11

Figure 1-7. Schematic representation of the media milling process..............................11

Figure 1-8. Schematic diagram of homogenization process....................................12

Figure 1-9. Scheme of typical three-phase diagram under isothermal condition...........15

Figure 1-10. Schematic diagram of cyclodextrin-drug complex................................. 17

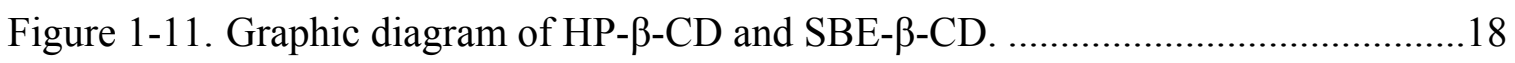

Figure 2-1. Scheme of solid dispersion classification system and new techniques.........21

Figure 2-2. Schematic flow chart of hot melt extrusion process. .................................23

Figure 2-3. Schematic diagram of components of a single screw extrusion. .................24

Figure 2-4. Scheme of solid dispersion preparation using spray drying technique.........29

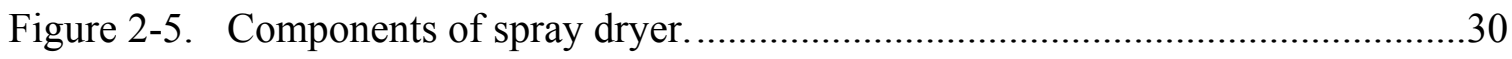

Figure $3-1$. Aqueous solubility of controls.........................................................44

Figure 3-2. Aqueous solubility of amorphous solid dispersions. ...............................45

Figure 3-3. Aqueous solubility of suspension solid dispersions and physical

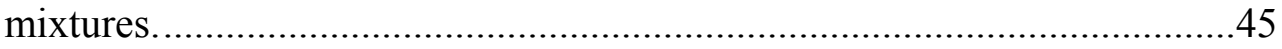

Figure 3-4. Scheme of reaction between furosemide and aqueous ammonia.................46

Figure 3-5. SEM pictures (part I) of furosemide powder and OSA starch...................48

Figure 3-6. SEM pictures (part II) of amorphous solid dispersion with furosemide to OSA starch at different ratio. 
Figure 3-7. XRPD spectrum (part I) of furosemide powder and OSA Starch.

Figure 3-8. XRPD spectrum (part II) of amorphous solid dispersion with furosemide to OSA starch at different ratio.

Figure 3-9. FTIR spectrum of furosemide, OSA starch and four controls.

Figure 3-10. FTIR spectrum of furosemide, OSA starch and solution solid dispersions.

Figure 3-11. FTIR spectrum of furosemide, OSA starch and suspension solid dispersions.

Figure 3-12. FTIR spectrum of furosemide, OSA starch and physical mixtures. .59

Figure 3-13. MDSC of control I (before and after treated with moisture).

Figure 3-14. MDSC spectrum of control II, III and IV.

Figure 3-15. Tg of OSA starch, amorphous solid dispersion 1:1, 1:2.3 and 1:9. .64

Figure 3-16. DVS plot of OSA starch and furosemide. .64

Figure 3-17. Water vapor sorption and desorption graph of control I. .65

Figure 3-18. DVS graph of solution solid dispersions.

Figure 3-19. Physical stability of solid dispersion at ambient condition and $40{ }^{\circ} \mathrm{C}$ .68

Figure 3-20. Physical stability (XRPD spectrum) of solution solid dispersion under $40{ }^{\circ} \mathrm{C} / 75 \% \mathrm{RH}$ for 2.5 months.

Figure 3-21. Dissolution profile of formulation I in SGF (pH 1.2) and phosphate buffer ( $\mathrm{pH}$ 5.8).

Figure 3-22. Dissolution profile of formulation II in SGF (pH 1.2) and phosphate buffer ( $\mathrm{pH}$ 5.8).

Figure 4-1. Phase-solubility diagrams of furosemide.

Figure 4-2. Scanning electron micrographs of furosemide, SBE- $\beta-C D$, and furosemide SBE- $\beta$-CD 1:1 sodid dispersion prepared by different methods.

Figure 4-3. FTIR spectra of (A) furosemide (B) SBE- $\beta-C D$ (C) KD solid dispersion (D) FD solid dispersion (E) SD solid dispersion and (F) physical mixture. 
Figure 4-4. XRPD spectrums of furosemide, SBE- $\beta$-CD, furosemide solid dispersions prepared with kneader, freeze drying and spray drying method and physical mixture.

Figure 4-5. DSC spectrum of SBE- $\beta-C D$, solid dispersions and furosemide...................89

Figure 4-6. Solid dispersion intrinsic dissolution profile. ............................................90

Figure 4-7. Solid dispersion tablet dissolution profile..................................................91

Figure 4-8. Dynamic sorption and desorption diagram of furosemide, SBE- $\beta-C D$, solid dispersions and physical mixture. .93 


\section{CHAPTER 1. BACKGROUND}

\subsection{Biopharmaceutics Classification System (BCS)}

The Biopharmaceutics classification system (BCS) was introduced in 1995 by Gordon L. Amidon et al. to correlate the drug in vitro dissolution performance to in vivo bioavailability (IVIVC) [1]. Two primary parameters: solubility and permeability were utilized as the basis for this drug classification system. In the system, four categories are applied to drugs: class I, high solubility, high permeability; class II low solubility, high permeability; class III, high solubility, low permeability; class IV, low solubility, low permeability.

In the system, the definition of high solubility is that the highest dose is soluble in $250 \mathrm{ml}$ or less of water over a $\mathrm{pH}$ range of $1-8$ at $37^{\circ} \mathrm{C}$. Permeability is defined as effective human jejunal wall permeability of a drug; the unit is $10^{-4} \mathrm{~cm}$ per second. High permeability means no less than $90 \%$ extent of absorption and at the same time, these drugs are basically stable in GI tract [2]. It is generally accepted that drug dissolution is the prerequisite for drug absorption for any orally given dosage form based on human GI tract physiological condition. For orally given drugs, passive transcellular diffusion is the major mechanism for drug absorption [3]. The mass of drug across the mucosal membrane of intestine is the upper limit amount that ready to enter system circulation [4].

\subsubsection{Relation between Do, Dn, An, and dissolution}

Besides solubility and permeability, three dimensionless parameters are also introduced to describe the relationship between dose, dissolution, drug solubility and drug permeability [4]. They are absorption number (An); dissolution number (Dn); and dose number (Do). Several equations are derived to describe the relation between those variables. For absorption number (An), it equals the permeability of drug divided gut radius and then times residence time or transit time of drug, see Equation 1-1.

$$
\text { Absorption number }(\mathrm{An})=\left(\mathrm{P}_{\mathrm{eff}} / \mathrm{R}\right) \cdot<\mathrm{Tsi}>
$$

Where $\mathrm{P}_{\text {eff }}$ is permeability, $\mathrm{R}$ is the gut radius and $<\mathrm{Tsi}>$ is the residence time of the drug within the intestine. Dissolution behavior is complex, for dissolution number (Dn), five variables are included in its equation: drug diffusivity, particle radius, solubility, density, and residence time, see Equation 1-2.

$$
\text { Dissolution number }(\mathrm{Dn})=\left(3 \mathrm{D} / \mathrm{r}^{2}\right) \cdot(\mathrm{Cs} / \rho) \cdot<\mathrm{Tsi}>
$$

In this equation, $\mathrm{D}$ is the diffusivity of the dissolved drug, $\mathrm{r}$ is the initial radius of the drug particle, Cs is the drug solubility, and $\rho$ is the density of the dissolved drug; $<$ Tsi $>$ is the same as in Equation 1-1. The concentration gradient of drug in gut is the original driving force for its diffusion which decided its diffusivity. Generally, the concentration 
of drug in gut is several orders higher than that across the membrane. Decreased particle size, increased solubility, prolonged GI tract residence time will increase the dissolution number [5]. A higher dissolution number is in favor of increase of drug absorption. For dose number (Do), it equals dose concentration divided solubility, see Equation 1-3.

$$
\text { Dose number }(\text { Do })=\frac{\mathrm{M} / \mathrm{V} 0}{\mathrm{Cs}}
$$

$\mathrm{M}$ is the dose of the drug and $\mathrm{V}_{0}$ is water needed to take the dose, generally 250 $\mathrm{ml}$ is set as default. Cs is drug solubility. For a given dose, increasing the water amount to take the drug or increasing drug solubility will reduce the dose number which favors of drug absorption.

By analysis and summarization of permeability and fraction absorbed of 18 drugs, (see Figure 1-1) [1], the relationship between drug fractions of absorbed and permeability is described as Equation 1-4.

$$
\mathrm{Fa}=1-\mathrm{e}^{-2 \mathrm{An}}
$$

In this equation, the assumption is that the drug is soluble in the GI tract. Basically, it applies to class I and class III compounds. In this case, absorption number (An) and permeability are two primary parameters to predict oral absorption. The absorption number (An) can also be expressed as drug residence time in intestine divided by drug absorption time. See Equation 1-5, which is derived from Equation 1-1. In Equation 1-1, the unit of $\mathrm{P}_{\text {eff }}$ is $\mathrm{cm} / \mathrm{s}$ which likes velocity; $\mathrm{R}$ is radius of gut, which likes distance; since time equals distance / velocity, the time of drug absorption equals radius of gut / permeability that is the reciprocal of $\mathrm{P}_{\text {eff }} / \mathrm{R}$, too.

$$
\text { Absorption number }(\mathrm{An})=<\mathrm{Tsi}>/<\mathrm{Tabs}>
$$

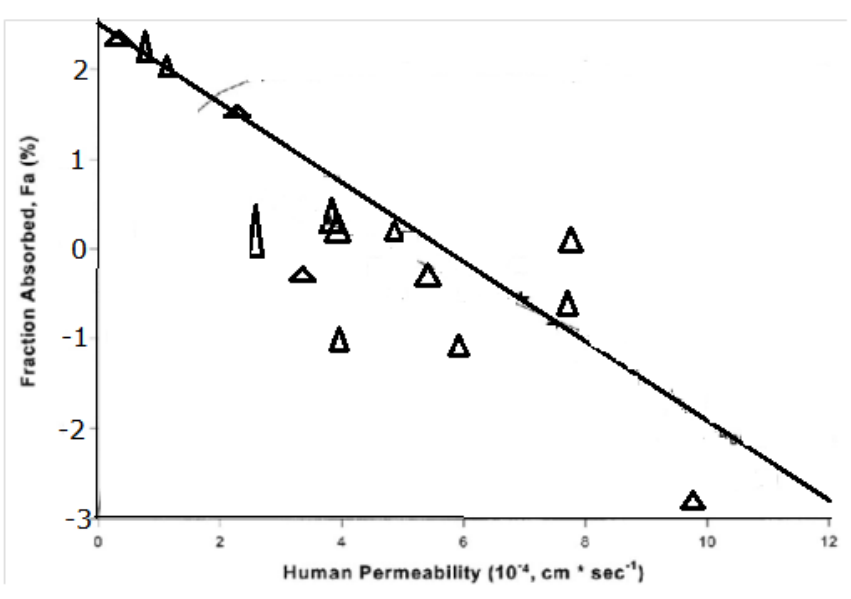

Figure 1-1. Correlation between human permeability and fraction dose absorbed. 
When residence time $\left(\mathrm{T}_{\mathrm{si}}\right)$ equals absorption time $\left(\mathrm{T}_{\mathrm{abs}}\right)$, absorption number $(\mathrm{An})$ is 1 from Equation 1-5, substituting $A n=1$ in Equation 1-4, Fa is calculated around 86\%. Return to definition of high permeability, Fa should be no less than $90 \%$. If calculating An from Equation 1-4, it will be bigger than 1. The physical meaning of An $>1$ is that drug absorption time is shorter than residence time. As long as the drug dissolves in the GI tract, the drug will be absorbed. The above discussion is applied to BCS class I and III compounds.

For BCS class II compounds, those drugs are poorly water soluble and GI fluid is not enough to completely dissolve them. In this case, Equation 1-6 is derived to describe the relationships between solubility, permeability, dissolution and drug absorbed $[3,4]$. Since Fa always equals or less than 1 , Do $\geq 2 \mathrm{An}$.

$$
\mathrm{Fa}=2 \mathrm{An} / \mathrm{Do}
$$

The concentration of these drugs in GI tract is equal to their solubility. The assumption of Equation 1-6 is dissolution rate should be faster than the rate of drug absorbed across the intestine membrane. Dissolution rate is the limited factor for these drugs oral absorption. For class II drugs, dissolution number (Dn) is commonly less than one.

At a given absorption number An, the relation between Do and Dn can be described by Figure 1-2 [1]. In Figure 1-2, An is fixed as 10 which means the drug is high permeability. Dose number (Do) and dissolution number (Dn) decide drug fraction of absorbed. From Figure 1-2, we can see that a low dose number and high dissolution number nearly always gave higher drug absorption. The change in Dose number is decided by water volume and solubility of drug. More water to take drug can decrease Do but this tool is limited by patient compliance and stomach volume capacity; increasing drug solubility can be a way; dose number is not commonly changed which is decided by physicochemical properties of compound and also decided by its pharmacodynamic and pharmacokinetic properties. For dissolution number (Dn), the higher dissolution number will result in higher fraction of drug absorption. Some formulation strategies can be applied: decrease particle size or increase dissolution rate. Two examples are shown in Figure 1-2. For digoxin, the Dn is between 0.1 1.0, Do is around 0.4 and the fraction drug absorption is around 0.4. Digoxin is a low dose drug, $0.5 \mathrm{mg} / \mathrm{dose}$, when particle size is decreased sufficiently, the dissolution rate increased so that the tablets and its hydroalcoholic solution were bioequivalent. But for piroxicam, Do is around 12 and Dn is less than 0.1 , its Fa is less than 0.2. To dissolve the dose, $2857 \mathrm{ml}$ water is needed. For this drug, solubility is the limitation for drug absorption. Enhancment of solubility can provided sufficient drug amount for absorption [6, 7].

\subsubsection{Determination of solubility}

Compound aqueous solubility can be seen as a capacity to form hydrogen bonds with water molecules. Or it can be seen as how easily its constituent atoms are 


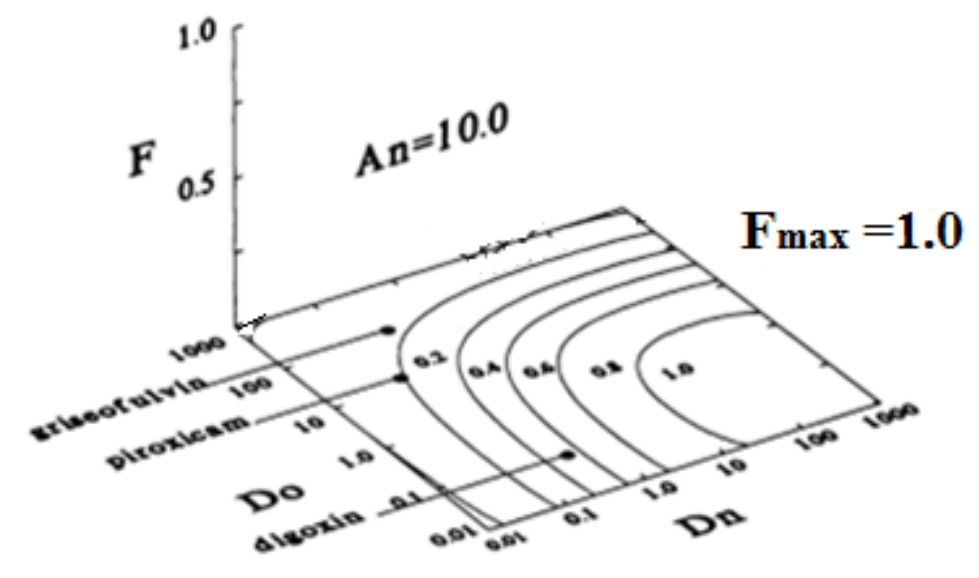

Figure 1-2. Relationship between Dn, Do and An.

surrounded (form hydrogen bonds) by water molecular network. Solubility is measured when an excess amount of drug is dissolved in aqueous media, which can be water for neutral drug or buffer for weak acid or base, at a certain temperature and pressure [4]. Analysis is commonly an HPLC or other specific method. The common definition of the degree of solubilization is described as in USP:

- Very soluble: Less than 1 part solvent needed to dissolve 1 part solute

- Freely soluble: From 1 to 10 parts solvent needed to dissolve1 part solute

- Soluble: From 10 to 30 parts solvent needed to dissolve 1 part solute

- Sparingly soluble: From 30 to 100 parts solvent needed to dissolve 1 part solute

- Slightly soluble: From 100 to 1000 parts solvent needed to dissolve 1 part solute

- Very slightly soluble: From 1000 to 10,000 parts solvent needed to dissolve 1 part solute

- Practically insoluble: More than 10,000 parts solvent needed to dissolve 1 part solute

\subsubsection{Determination of permeability}

After drug dissolution in the GI tract, it is ready for crossing the gut membrane. The drug mass crossing membrane would subsequently enter the blood. The permeability indicated how easily and how much of drug crosses membrane in a given time. An In vitro cell line method and in vivo or in situ intestinal perfusion method are accepted by regulatory agency for determination of permeability [8]. Both methods need compound with known permeability as internal standard or reference drug.

The Caco-2 model is widely accepted and applied to measure in vitro permeability. The cell line Caco-2 is from human colonic cancer cells and used in transport system research from the 1970s [9]. It can differentiate and form polarized 
monolayer with tight junction between cells. These properties are similar with that of intestinal cells. Moreover, Caco-2 cells have several transport systems: including amino acid, vitamin B, glucose, bile acid, small peptide transporters and P-glycoprotein efflux system. Figure 1-3 (Modified from Ref. [10]) shows a Caco-2 cell permeability model. A drug or reference solution is added to the upper compartment, and then incubated for hours. A sampling point is set to derive a mass transport per time $(\mathrm{dm} / \mathrm{dt})$ curve for permeability calculations. For the Caco-2 permeability calculation, see Equation 1-7.

$$
\mathrm{P}_{\mathrm{eff}}=(\mathrm{dm} / \mathrm{dt}) /\left(\mathrm{A} \times \mathrm{C}_{0} \times 60\right)
$$

In this equation, $\mathrm{P}_{\text {eff }}$ is the effective permeability in $\mathrm{cm} / \mathrm{sec}, \mathrm{m}$ is mass across the membrane, $A$ is surface area $\left(\mathrm{cm}^{2}\right)$ for transport, $\mathrm{C}_{0}$ is the slope of best fit line for the curve $\mathrm{dm} / \mathrm{dt}, \mathrm{C}_{0}$ is the initial drug concentration $(\mu \mathrm{M})$. If the $\mathrm{P}_{\mathrm{eff}}$ of a drug is higher than $2 \times 10^{-4} \mathrm{~cm} / \mathrm{s}$, then complete drug absorption will be considered, otherwise, drug permeability is low and the absorption is incomplete[11].

Another widely used method is in situ single-pass (closed loop) rat perfusion model. Similar toCaco-2 cell model, an internal standard or reference compound is also required. See Figure 1-4, from Ref. [12] for in situ perfusion rat model diagram.

The segment of small intestine (about $6-8 \mathrm{~cm}$ ) is cut from gut with fully blood supply. One end is cannulated to allow for drug injection by syringe. The other end is connected with a tube for sample collection. The remaining drug in the intestine is collected in a sample vial for concentration measurements by LC-UV or LC-MS. The two ends of remaining intestine are tied off by a silk suture. The rat moves freely in a restraining cage during experiment. After experiment, the animals are euthanized. This model is accepted by the FDA as a tool to determine permeability, regional disposition,

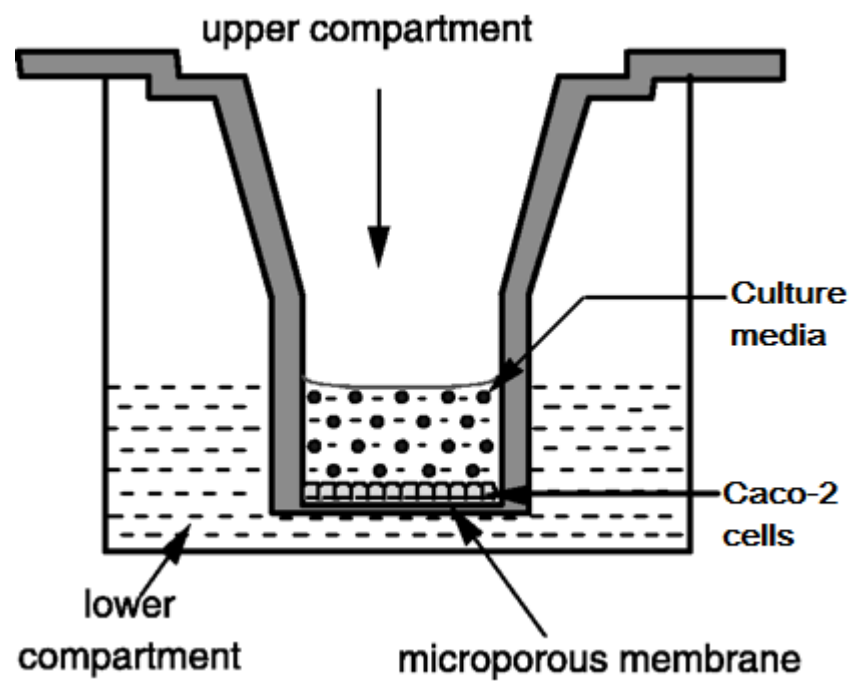

Figure 1-3. Caco-2 cell permeability model diagram. 


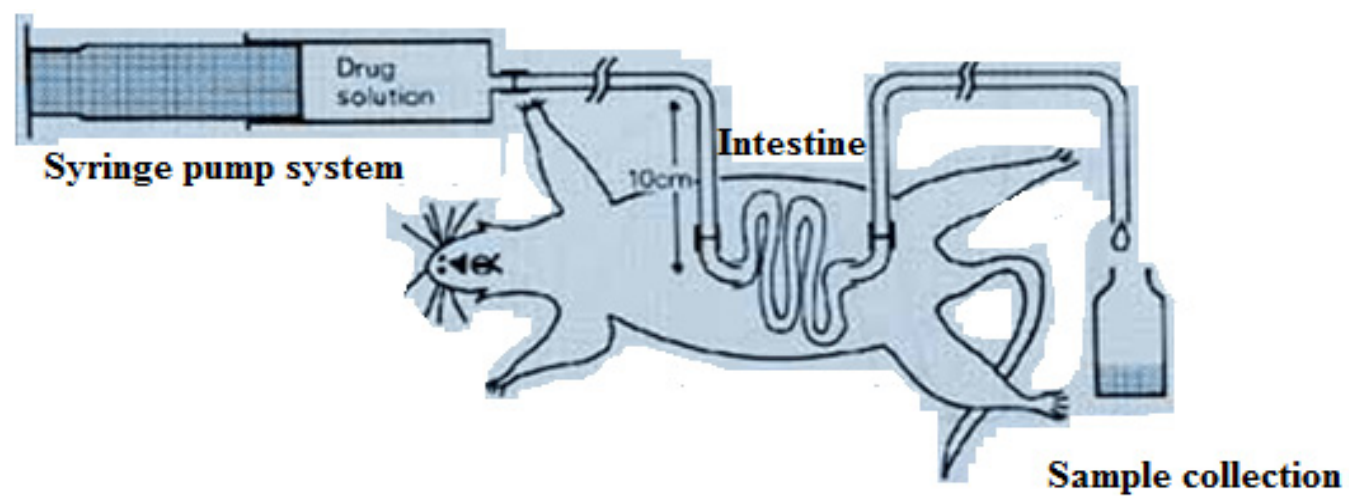

Figure 1-4. Intestinal perfusion model.

mechanisms of absorption and excretion of drugs, etc [9]. The Permeability calculation for this model is shown in Equation 1-8.

$$
\mathrm{P}_{\mathrm{eff}}=\left[-\mathrm{Q}_{\text {in }} \ln \left(\mathrm{C}_{\text {out }} / \mathrm{C}_{\text {in }}\right)\right] / 2 \mathrm{rL}
$$

Where $\mathrm{C}_{\text {out }}$ and $\mathrm{C}_{\text {in }}$ correspond to the drug concentration out of the intestine and the drug concentration flowed in intestine respectively. While $r$ is the rat intestinal radius which is set to $0.18 \mathrm{~cm}$; $\mathrm{L}$ is the length of isolated intestine, commonly $6 \sim 8 \mathrm{~cm} \mathrm{[13].} \mathrm{Q} \mathrm{is}$ the flow rate through the intestine.

Sometimes, for generic drug applications, sponsors provided permeability determined by Caco-2 model and in the situ single-pass (closed loop) rat perfusion model. The results from two models can be similar in some cases, but also can be different. The advantage of the Caco-2 cell model is its cost savings: the cell culture is cheaper than animal studies and the experiment can be designed for high throughput compound screening. The disadvantage includes Caco- 2 cells that are from a colonic cell line that is differentiated into small intestinal -like tissue. Actually its tight junction between cells is more like colonic cells than small intestine cells which can cause a permeability under-prediction for low absorption drugs [14]. Secondly, it may not express proper transporters as a true small intestine cells would be expected to.

As a result of disadvantages in Caco-2 model, the more accurate in situ rat perfusion model is often introduced for permeability determinations. Thus, for biowaiver purpose, sponsors may provide animal permeability result as supplement if the Caco-2 model under-predicts permeability [9].

\subsubsection{Determination of dissolution}

The priority condition for oral drug absorption is the dissolution of drug at the site of absorption. The Noynes-Whitney equation (Equation 1-9) described the variables that can affect drug dissolution: 


$$
\mathrm{dm} / \mathrm{dt}=\mathrm{DS}(\mathrm{Cs}-\mathrm{Ct}) / \mathrm{Vh}
$$

In the equation, $\mathrm{dm} / \mathrm{dt}$ is the dissolution rate, which means the change in the amount of drug dissolved (m) per unit time ( $\mathrm{t}$ ); D is the diffusion coefficient which related to temperature, solute radius and viscosity of diffusion medium. $\mathrm{S}$ is the surface area; $h$ is the thickness of the diffusion film adjacent to the dissolving surface; Cs is the saturation solubility of the drug molecule; $\mathrm{Ct}$ is the concentration of the dissolved solute; and $\mathrm{V}$ is the volume of the dissolution medium. From the equation we can see, increasing surface area as particle size decreases, or increasing saturation solubility can both increase dissolution rate [15]. If dissolution of drug is faster than its gastric emptying, (this point is true for BCS class I drugs), basically, dissolution won't influence the bioavailability of drug. For this type of drugs, they can express the similar bioavailability but their dissolution can be different. In this case, the gastric emptying rate will be the limiting factor for drug bioavailability (BA). For BCS class I drug, in vivo in vitro correlation (IVIVC) could be constructed [16].

For BCS class II drugs with low solubility and high permeability, dissolution rate and extent can both be the rate-limiting factor to drug absorption. Dissolution rate can be increased by decreasing particle size; where extent of dissolution increase depends on solubility enhancement which can be introduced by many new techniques besides adding surfactant in formulation.

- Class I: High Solubility - High Permeability

- Class II: Low Solubility - High Permeability

- Class III: High Solubility - Low Permeability

For BCS class III drugs with low solubility and high permeability, they are easily soluble as class I drug; it is gastric emptying rate rather than dissolution rate that affect more on BA. The low permeability is the limiting factor for their oral absorption. If the drug absorption occurs as linear process, increasing dose is a way to increase bioavailability. Or if P-glycoprotein pumped the drug out of membrane, then the Pglycoprotein inhibitor may be added in formulation to inhibit its efflux function [3].

Drugs with low solubility and low permeability are categorized to BCS class IV. These compounds have poor oral bioavailability and are not easy to develop oral dosage form. Generally, non-oral dosage form will be considered for BCS class IV compound. However, there are still few oral BCS class IV drugs on market: hydrochlorothiazide, taxol, and furosemide.

For oral dosage forms after administration, the drug is passed into stomach, disintegrates here, then drug dissolves to allow absorption. Simulated gastric fluid (SGF) is commonly used as a dissolution media. Since the small intestine is the major area for most drug absorption, simulated intestine fluid (SIF) is another commonly used dissolution media. When developing dissolution method for BCS class I or class III drugs, a volume of $900 \mathrm{ml}$ can provide sink condition for those drugs dissolution; surfactant maybe added in SGF or SIF to make sink condition for BCS class II or class 
IV drugs. Besides the aforementioned dissolution media, other bio-relevant media are prepared for mimic fast state gastric condition, fed state gastric condition, fast state intestine condition and fed state intestine condition. In the dissolution media, surfactants and enzymes are often also selected as needed [17].

Basket (USP apparatus I) or paddle (USP Apparatus II) dissolution is the most commonly used methods for oral dosage form. The volume of media is generally set to $900 \mathrm{ml}$. For high soluble BCS class I and III compounds, the duration time should be shorter than that of BCS class II or IV. For IVIVC purpose, the dissolution criteria is set by FDA as no less than $85 \%$ drug substance is dissolved in three different dissolution media (SGF with pH1.2 without enzyme, $\mathrm{pH} 4.5$ buffer and SIF or pH6.8 buffer without enzyme); for each formulation, 12 dosage units are evaluated. Sampling points should be enough to derive the dissolution curve for comparison [18]. The variance of initial point on dissolution curve (like $10 \mathrm{~min}$ point) should be less than $20 \%$, the following points should be within $10 \%$ variance. The similarity factor $(f 2)$ is required to compare the dissolution profiles. See Equation 1-10 for $f 2$ value calculation.

$$
f 2=50 \cdot \log \left\{\left[1+\frac{1}{n} \sum_{t=1}^{n}(R t-T t)^{2}\right]^{-0.5} \times 100\right\}
$$

Where $\mathrm{n}$ is the number of time points, $\mathrm{Rt}$ is the mean cumulative percent dissolved of a reference drug at time $t, T_{t}$ is the mean cumulative percent dissolved of a test drug product at time t. If no less than $85 \%$ drug dose dissolved within $30 \mathrm{~min}, f 2$ value comparision is not necessary. If $85 \%$ drug amount dissolution is longer than $30 \mathrm{~min}$, then $f 2$ value may be calculated to compare dissolution profiles. Two dissolution profiles would be considered similar if $f 2 \geq 50$ and the difference of two dissolution profile is less than $10 \%$ [19].

\subsection{Formulation Strategies for Poorly Water Soluble Drug Development}

Currently, BCS is widely accepted as a must tool in new drug or generic drug development. In the early stage of drug development, solubility and permeability of lead compounds are measured to decide which category the drug belongs to. Then a formulation strategy has been previously used successfully might be considered for this compound. For class I compounds, they are easily soluble and have good absorption, formulation for these compounds is more likely to be a stability issue than a dissolution problem. For class II compounds, a formulation strategy is critical to improve dissolution rate if the limiting-step of absorption is dissolution rate or improve solubility if the drug is solubility-limiting oral absorption. Micronization, nanotechnology, cyclodextrin complexation, lipid based formulation, micellization, microemulsions and solid dispersion are accepted approaches to handle poorly solubility issues. It is not easy to develop BCS class III compounds because it is hard to change permeation of drug and

membrane. Some permeation enhancers have been studied for this purpose [20-22]. For BCS class IV compounds, approaches for improving solubility and permeability are needed at the same time, plus it is necessary to understand the compound absorption mechanism and factors affecting bioavailability. Alternative delivery methods, such as 
i.v. or it may be necessary to go back to lead chemical optimization. Nevertheless, several Class IV drugs do exist. Examples include taxol, cyclosporin A and furosemide. In these innovative formulation strategies, nanotechnology and lipid based formulations will be discussed in the following subtopics, as well as cyclodextrin complexation. Solid dispersion approachs will be discussed in details in Chapter 2.

\subsubsection{Nanotechnology in poorly water soluble drug development}

Particle size reduction is an effective tool to enhance the dissolution rate of poorly soluble drugs. It is well known that the micronization can increase in vivo absorption and bioavailability of poorly water soluble drugs. But micronization works for those drugs that oral absorption is limited by dissolution rate. The micro-scale particles are not small enough to increase oral bioavailability of those drugs which oral absorption is limited by solubility, such as high dose BCS class II drugs. But in the case of reducing particle size to nano-scale, the oral BA of aforementioned two type poorly soluble drugs can both be enhanced [23]. Some nanosuspensions on market or in clinical trials are listed in below.

Nanosuspensions we will discuss here is a colloidal dispersion of pure drug particles in water with other necessary ingredients. The particle size for nanosuspension is generally smaller than $300 \mathrm{~nm}$ for Brownian motion to keep system stable. Individual particle in a nanosuspension cannot be seen by the naked eye. The drug particle in nanosuspersion is in a preferred crystalline form with sufficiently small size. Usually, the preferred API for this formulation is those insoluble drugs with solubility less than 0.1 $\mathrm{mg} / \mathrm{ml}$. If the solubility is higher than $0.1 \mathrm{mg} / \mathrm{ml}$, other strategies including co-solvent, surfactant, emulsion, etc. might be utilized [24].

\subsubsection{Formulation rationale and ingredient function}

The constituents of a typical nanosuspension include drug (API), stabilizers, surfactants and water. Basically, the nanosuspension is a thermodynamically stable system with high energy. The preparation process is a surface area increase process which will induce free energy increase. But adding surface-active agents can compromise the surface change effect by decreasing interfacial tension, thus keep or decrease free energy. This basic principle can be shown Equation 1-11, where $\Delta \mathrm{G}$ is change of free

$$
\Delta \mathrm{G}=\gamma_{\mathrm{s} / 1} \bullet \Delta \mathrm{A}
$$

energy, $\gamma_{\mathrm{s} / 1}$ is the interfacial tension between solid (particle) and liquid (water), $\Delta \mathrm{A}$ is the change of surface area. Generally, two type surface-active agents: ionic surfactant and non-ionic surfactant are used in nanosuspensions [25]. Function of polymer and surfactant in preparation and stabilization of nanoparticles is shown in Figure 1-5, modified from ref. [25]. 


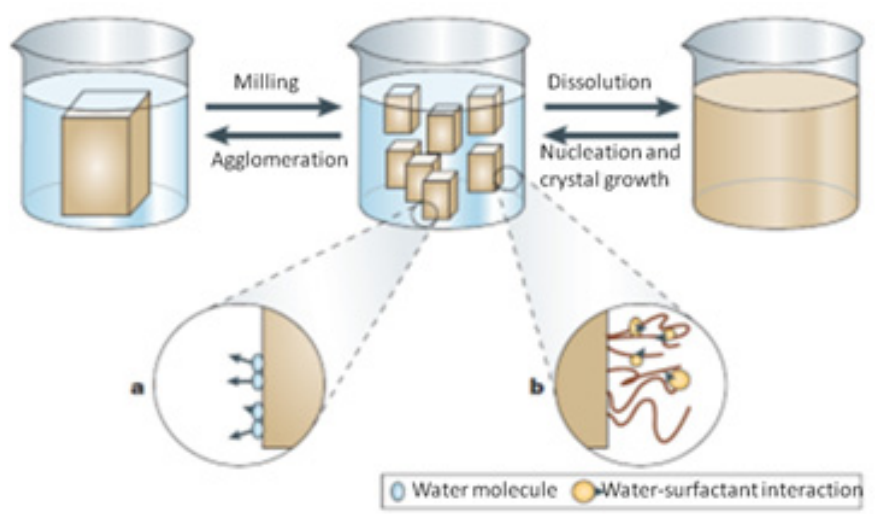

Figure 1-5. Function of polymer and surfactant in nanoparticles.

In Figure 1-5, for the case of circle a, hydrophobic crystal surface directly contacting water molecules leads to crystal agglomeration. For the case of circle b, polymer and surfactant synergy stabilized crystal surface, the stabilizers not only reduced contact opportunities between water and crystal particles, but also decreased interfacial tension by attraction between water and surfactant. In a nanosuspension, two close nanoparticles tend to agglomerate because of Van Der Waals attractive forces or hydrophobic attraction, but with a polymer and surfactant in the system, the trend can be reduced by steric repulsion or electrostatic repulsion from exicipient.

This point can also be interpreted as polymer molecules inserted into API particles thus increasing the distance of two hydrophobic particles and decreasing their hydrophobic attraction. When adding ionic surfactants, these molecules attached at the interface of water and nanoparticles to not only reduce the interfacial tension $\left(\gamma_{\mathrm{s} / 1}\right)$, but also incur static repulsion to prevent crystal growth and particle agglomeration.

Commonly used polymer and surfactant include cellulose derivatives; poloxamer, polysorbates, transcutol, etc. are shown in Figure 1-6. Selection of exicipients will be according to the properties of API [26].

\subsubsection{Nanosuspension preparation methods}

The first successful scale-up nanosuspension manufacture technique is owned by Elan Drug Delivery. It is media mill. The mechanical diagram is shown in Figure 1-7.

The API particles (after premilling by ball mill or micronized API) are mixed together with selected surfactants and polymer in water, then place the suspension in the chamber which is charged by milling media, a drive shaft attached to rotating disk to make it rotate at a very high shear rate. A cooling system is included to make the whole process under controlled temperature. In process, particle size is determined by stress intensity and the number of contact points between API crystal and media grinding 


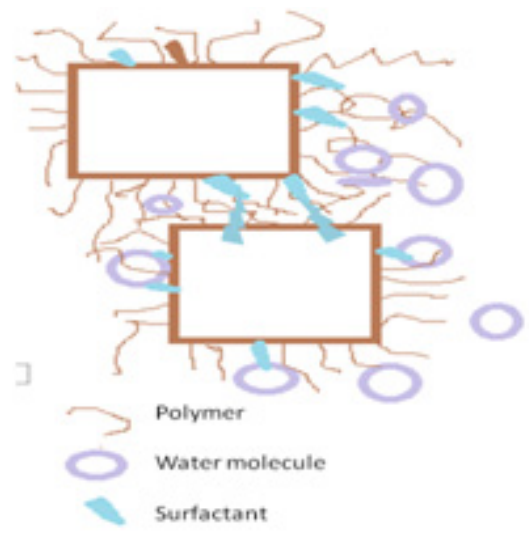

Figure 1-6. Relation in nanoparticles, polymer, surfactant and water molecules.

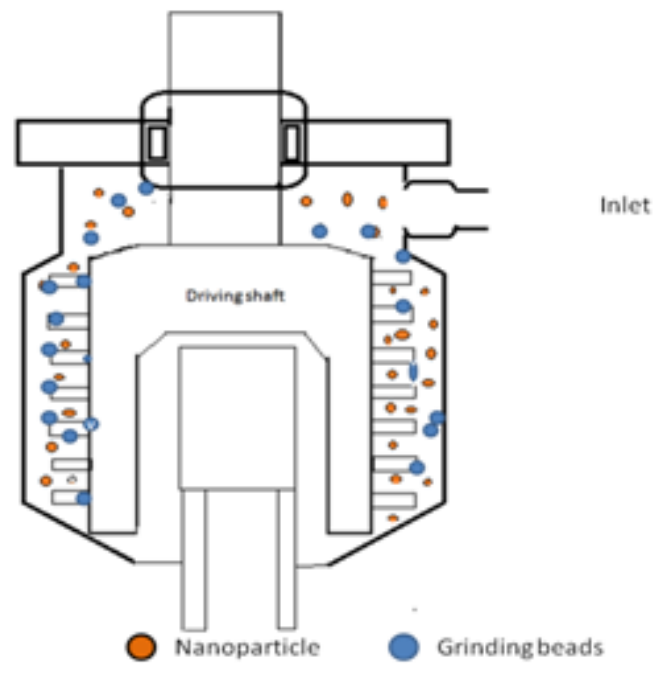

Figure 1-7. Schematic representation of the media milling process. 
beads. After milling $30 \sim 60 \mathrm{~min}$, the particle size can be reduced to $200 \mathrm{~nm}$ with narrow distribution. The variance among batches is very small $(\leq 2 \%)[23]$.

High pressure homogenization is another technique that applied to prepare nanosuspension from lab to manufacture scale. The schematic diagram of this piston-gap homogenizer is shown in Figure 1-8. For this method, pre-suspension is necessary before preparing nanosuspension. The pre-suspension, usually a micronized, particle size which can be smaller than $5 \mu \mathrm{m}$, placed in the chamber to pass through a narrow channel (homogenization gap), the flow velocity is increased tremendously under high pressure (1400 bar). According to Bernoulli principle, simultaneously static pressure of presuspension fluid decreased which reduced the boiling point of water, then water start to evaporate to form bubble in the boiling area. At the same time, the pre-suspension goes through the channel (e.g. $25 \mu \mathrm{m})$, then water bubble implodes when fluid leaves channel due to the pressure change (cavitations effect). The energy produced from cavitations' cracked the fluid into nanoparticles. In this method, suspension needs to be micronized in case big particles occlude the narrow channel. Homogenization pressure and numbers of homogenization cycles are keys for final product quality $[23,27,28]$.

Other methods include crystal precipitation, emulsion or microemulsion as templates to prepare nanosuspensions. The drug will be first dissolved in a solvent or cosolvent, then form nuclei at slow speed by adjusting temperature, then optimizing temperature for crystallization at a fast speed to keep the size of crystals in nano-scale. During this crystal precipitation process, the control of temperature is the key. When preparing nanoparticles from emulsions or microemulsions, the first step is to form a stable emulsion, then agitate water phase under high stirring speed, the solvent evaporated, solid particles obtained. Surfactants are necessary in the process [28].

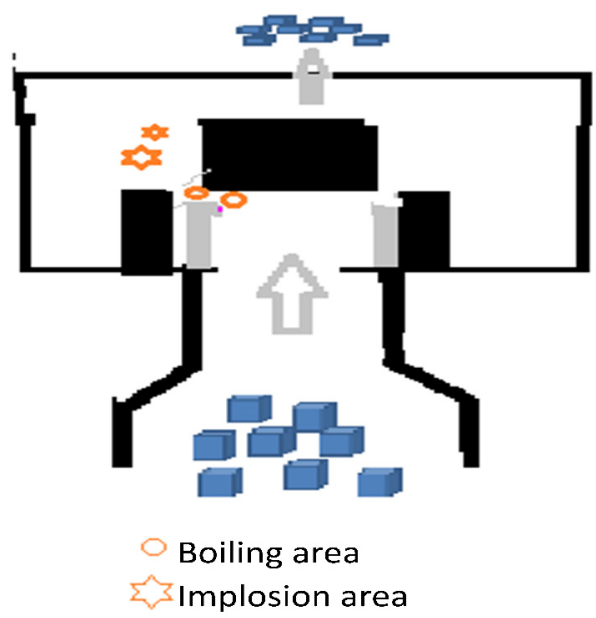

Figure 1-8. Schematic diagram of homogenization process. 


\subsubsection{Characterization and key factors affecting product quality}

Particle size distribution, stability and bioavailability are three critical factors affecting the quality and efficiency of nano-suspensions. Laser light diffraction is used to analyze the drug substance in aqueous nano-suspensions. In data calculation, Fraunhofer and Mie therories are employed. The former one is the default for particle size calculation. In the USP, if particle size is smaller than $25 \mathrm{um}$, Mie theory can also be applied. If inconsistent results occurred, other techniques such as scanning electronic microscopy (SEM) and Mie theory will be applied. XRPD is also used to characterize the solid form or polymorph. Stability is tested under the accelerated condition to see if agglomeration occurred, shipping conditions are also evaluated, too. Bioavailability is determined by conducting bioavailability and pharmacokenetic studies; $\mathrm{C}_{\max }, \mathrm{T}_{\max }$ and AUC are compared to reference formulations [29, 30].

\subsubsection{Commercial products and promising drug product status}

Currently commercial products include tablet, suspension and capsule. Rapamune is an immune-suppressant tablet based on Elan nano technique which is manufactured by Wyeth. Merck also has one nanoparticle product: Emend which is anti-emetic drug in capsule form applying Elan nano technique too. In addition to aforementioned drugs, there are several drugs on every stage of clinical trial [31].

\subsubsection{Self-emusifying drug delivery system}

Several type of drug formulation system can be categorized as lipid based drug delivery systems. These formulation approaches also target to deliver poorly water soluble drugs. Self-emusifying drug delivery system (SEDDS) is one of these approaches. Excipients including oil, co-solvent and surfactant are mostly often considered in this system. The mixture of oils, surfactant, and often drug co-solvents are supposed to be isotropic. When the whole system contacts with water in vitro or in vivo, the oil in water emulsion will be formed and drug is released [32].

\subsubsection{Basic principle and excipient function}

The exactly mechanism of how the system works is still a remaining work for discover. But commonly reorganization is the relationship among liquid crystal formation, oil-water phase inversion temperature, the increased water solubility by lipid formulation that affects the self-emusifying efficiency. The equilibrium phase diagram is the priority part for the SEDDS development. The oil is the most important ingredient in the SEDDS which is used as drug solvent and oil phase, sometimes, a co-surfactant will be selected to work together with oil to dissolve more drugs. Both long and medium chain triglyceride oils are candidates. In SEDDS, food oils are not frequently used due to the low efficiency in dissolving API and long term stability issue [33]. 
The most commonly used surfactants in SEDDS are non-ionic surfactants which have high hydrophilic-lipophilic balance (HLB). Ethoxylated polyglycolyzed glycerides and polyoxyethylene 20 oleate (Tween 80 ) are two types of widely used surfactants. The usual amount of surfactant in SEDDS is about $25 \sim 60 \%$. The high usage amount of surfactants may induce a toxicity problem which is a major concern in surfactant selection. Even with Tween 80, it may cause GI irritation when used in long chronic application. Considering the toxicity issue; the choice of surfactant will be generally limited to the aforementioned two types. The type and amount of surfactant are two critical parameters to develop a good formulation. In SEDDS, surfactant is incorporated in the interface of oil and water, to help form micelles or fine-emulsion. With surfactant, the interfacial tension between water and oil can reach ultra-low values which will benefit drug stabilization in oil and fast dispersing when met with large amount water or fluid, such as in the GI tract [34].

The co-solvent is also a critical ingredient in SEDDS. Propylene glycol (PG), and polyethylene glycol (PEG) are two poplar so-solvents. It helps to dissolve API and quickly disperse SEDDS to form fine oil in water emulsion in drug dissolution and absorption.

\subsubsection{Phase diagram and characterization tools}

A phase diagram is the first step to develop a SEDDS. Usually the constituents of oil, co-solvent, surfactant and water without drug will be first tested at room and physiogical temperature to construct a phase diagram. An initial ratio between oil, cosolvent and surfactant will be assigned empirically as a starting point; the proper ratio among of oil, cosolvent and surfactant will be decided from the first phase diagram. Then the optimized ratio between oil and cosolvent is set as one component to construct second three phase diagram or pseudo three-phase diagram. A typical three-phase diagram is shown in Figure 1-9. A series of screening experiments is necessary to construct it. The triangle line is single phase line; the line inside is two components equilibrium line, two components are miscible in this line; the gray area inside triangle is the miscible area of three components which is what we need. Basically, the mix ratios of components in the gray area can be selected to further incorporate drug. According to drug dose and loading amount, the ratio among oil, co-solvent and surfactants would be adjusted $[32,35]$.

Upon dissolution, when SEDDS meets water or fluid in GI tract, it should disperse quickly and form fine oil in water emulsion. The co-solvent in the system assists the quick dispersion and large amount of surfactant is quickly distributed at the interface between oil and water to decrease the interfacial tension. Drug is kept in oil and ready for absorption $[33,36]$.

Upon dissolution or when the drug entered GI fluid, precipitation problems happen occasionally this is the intrinsic property of SEDDS. In that case, phase equilibrium is broken and drug can precipitate out. Phase break can be induced by temperature change, such as temperature changed from room to physiological condition, 

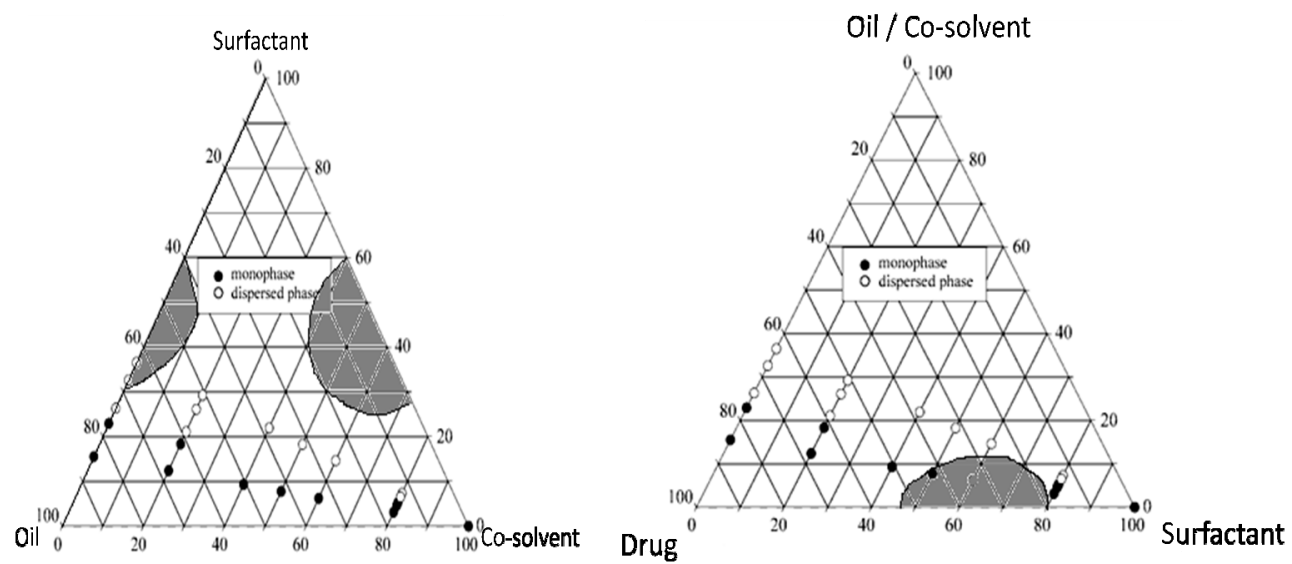

Figure 1-9. Scheme of typical three-phase diagram under isothermal condition.

cloud point reached; or induced by inability of the system to contain the drug under the in vivo conditions.

\subsubsection{Commercial products}

Several marketed products are applied SEDDS in their formulation and clinical practice shown benefit over side effect of high amount of surfactant. These drugs are summarized in Table 1-1, including major ingredients of each formulation $[37,38]$.

\subsubsection{Cyclodextrin application in drug delivery}

Cyclodextrin are cyclic oligosaccharides of glucose. Usually when one molecular cyclodextrin contains 6,7 or 8 glucose molecules, these oligomers will be referred to as $\alpha, \beta$ or $\gamma$ cyclodextrin. The cylinder shape molecules have amphiphilic properties that can form water soluble complex with small molecules and specific portion of large molecules. The shape and complex of cyclodextrin diagrams are shown in Figure 1-10.

The outside of cylinder is hydrophilic which incur the water solubility to cyclodextrin, the inside cavity is hydrophobic which form complex with poorly water soluble drug, thus increase the apparent solubility of drug, then further increase the bioavailability. It can also benefit the drug stability, prevent degradation of compound $[39,40]$.

The first cyclodextrin was discovered in 1891 and the first patent of cyclodextrin in drug formulation was issued to Freudenberg et al. in 1953. After that, the research on cyclodextrin became hot area for some years. During these years' study, the chemical properties including determination method, molecular structure characterization, drug 
Table 1-1. Summary of commercial SEDDS drug products.

\begin{tabular}{|c|c|c|c|c|c|c|c|}
\hline Trade name & Generic name & Dosage form & Surfactant & Solvent & Oil & Company & $\begin{array}{c}\text { Approval } \\
\text { date }\end{array}$ \\
\hline Sandimmune ${ }^{\circledR}$ & Cyclosporine A & $\begin{array}{l}\text { Soft gelatin } \\
\text { capsule }\end{array}$ & $\begin{array}{c}\text { Linoleoyl } \\
\text { macrogolglycerides } \\
\text { (polyoxylglycerides) }\end{array}$ & Alcohol & Corn oil & Novartis & 2-Mar-90 \\
\hline Neoral $^{\circledR} *$ & Cyclosporine A & $\begin{array}{l}\text { Soft gelatin } \\
\text { capsule }\end{array}$ & $\begin{array}{l}\text { Cremophor RH } 40 \\
\quad(H L B \text { 14 16) }\end{array}$ & Ethanol & $\begin{array}{l}\text { PG/Corn oil mono- } \\
\text { di-triglycerides/ } \alpha- \\
\quad \text { tocopherol }\end{array}$ & Novartis & 14-Jul-95 \\
\hline Norvir $^{\circledR}$ & ritonavir & $\begin{array}{l}\text { Soft gelatin } \\
\text { capsule }\end{array}$ & $\begin{array}{c}\text { Cremophor EL } \\
\text { (HLB 12 14 ) 13\% }\end{array}$ & $\begin{array}{c}\text { Ethanol } \\
12 \%\end{array}$ & Oleic acid 75\% & $\begin{array}{c}\text { Abbott } \\
\text { laboratories }\end{array}$ & 10 -Feb-10 \\
\hline Fortovase $^{\circledR}$ & Saquinavir & $\begin{array}{l}\text { Soft gelatin } \\
\text { capsule }\end{array}$ & NA & Povidone & $\begin{array}{l}\text { Medium chain } \\
\text { mono- and di- } \\
\text { glycerides, DL- } \alpha- \\
\text { tocopherol }\end{array}$ & $\begin{array}{l}\text { Hoffmann-La } \\
\text { Roche lnc. }\end{array}$ & 24-Dec-03 \\
\hline Agenerase ${ }^{\circledR}$ & Amprenavir & $\begin{array}{l}\text { Soft gelatin } \\
\text { capsule }\end{array}$ & TPGS (HLB 13) & PEG 400 & $\begin{array}{l}\text { PG (phosphatidyl } \\
\text { glycerol) }\end{array}$ & Glaxosmithkline & 5-Feb-02 \\
\hline
\end{tabular}

* Neoral ${ }^{\circledR}$ is not bioequvelent with Sandimmune ${ }^{\mathbb{R}}$, it has high bioavailability than Sandimmune ${ }^{\mathbb{R}}$. 

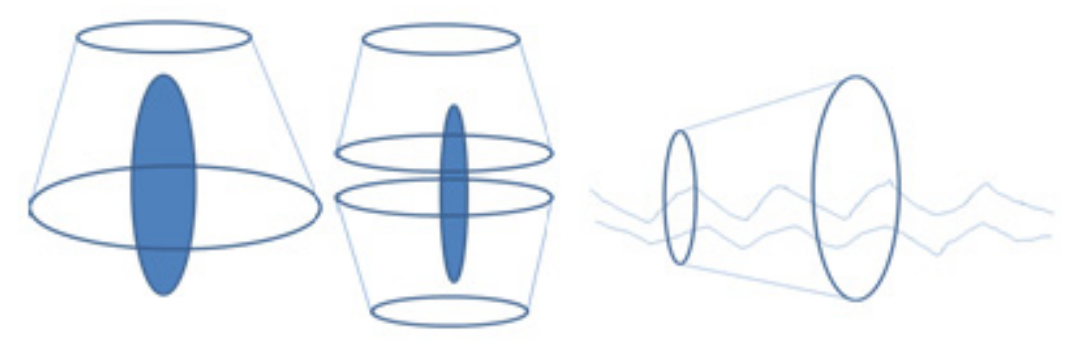

Figure 1-10. Schematic diagram of cyclodextrin-drug complex.

solubilization mechanism, complex form capacity are thoroughly disclosed. Besides chemical structure and function, biology, pharmacokenetics, pharmacodynamics and toxicity in human and animal are also studied. These researches found renal toxicity of cyclodextrin when administered i.v. Oral applications do not have renal toxicity since enzymes excreted from pancreas and stomach can degrade cyclodextrin. But fortunately, chemical modifications done to original cyclodextrins reduced toxicity and made it safe

to be used as a solubilizer for i.v. Modified cyclodextrin not only improved the safety but also the aqueous solubility. The solubility of $\alpha, \beta$ or $\gamma$ cyclodextrin in water at ambient temperature is $13 \%, 2 \%$ and $26 \% \mathrm{w} / \mathrm{w}$ respectively, whereas modified cyclodextrins have increased solubility which can reach to $60 \% \mathrm{w} / \mathrm{w}$ or even more. Currently, application of cyclodextrin have become a common solution for formulators to solve solubility problem of poorly water soluble drugs in various administration routes, including oral, ophthalmic, i.m., i.p., etc. Two modified $\beta$-cyclodextrins: hydroxypropyl- $\beta-C D$ (HP $\beta C D$; Figure 1-11) and sulphobutyl ether derivatives of $\beta-C D(S B E \beta C D$; Figure 1-11) are widely accepted as i.v. excipient by regulatory agency in many countries [41].

Cyclodextrin are successfully used as drug solubilizers and have made commercialization of many drugs possible. Table 1-2 summarized marketed drugs with cyclodextrins [39]. Cyclodextrins do have some disadvantages, such as low drug loading which limits its application to lower dose potent drugs and a relatively high price.

\subsection{Summary}

Based on BCS classification, drugss are divided into four categories. For class II and class IV, the drug solubilization is a critical issue for product development. The selection of formulation strategies can be various, from simple to complicated, from low to high price. The screening sequence can follow: co-solvent, alcohol, lipids; or new techniques, such as SEDDS, nanosuspension, solid dispersion. But for those new techniques, each has its own advantage and disadvantages. The benefit-risk or benefiteconomy would be evaluated for a decision. The solid dispersion technique will be discussed in detail in Chapter 2. 


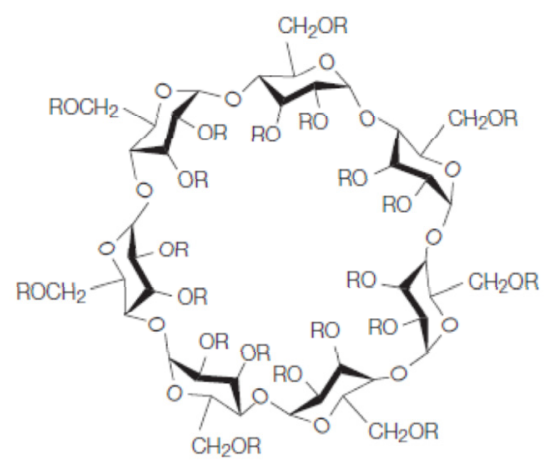

R: 2-Hydroxyisobutyl- $\beta$-cyclodextrin (HPBCD) $-\mathrm{CH} 2 \mathrm{C}(\mathrm{CH} 3) 2 \mathrm{OH}$ or $-\mathrm{H}$

Sulphobutylether- $\beta$-cyclodextrin (SBE $\beta C D$ ) $-(\mathrm{CH} 2) 4 \mathrm{SO} 3 \mathrm{Na}$ or $-\mathrm{H}$

Figure 1-11. Graphic diagram of HP- $\beta-C D$ and SBE- $\beta-C D$. 
Table 1-2. Summary of drug products using cyclodextrin.

\begin{tabular}{|c|c|c|c|}
\hline Drug & $\begin{array}{c}\text { Administration } \\
\text { route }\end{array}$ & Trade name & Market \\
\hline Alprostadil (PGE1) & Intravenous & $\begin{array}{l}\text { Prostavastin, } \\
\text { Caverject, Edex }\end{array}$ & $\begin{array}{c}\text { Europe, Japan, United } \\
\text { States }\end{array}$ \\
\hline Cefotiam hexetil $\mathrm{HCl}$ & Oral & Pansporin T & Japan \\
\hline $\begin{array}{c}\text { Limaprost } \\
\text { B-Cuclodextrin }(B C D)\end{array}$ & Oral & Opalmon, Prorenal & Japan \\
\hline Benexate $\mathrm{HCl}$ & Oral & Ulgut, Lonmiel & Japan \\
\hline Cephalosporin & Oral & Meiact & Japan \\
\hline Cetirzine & Oral & Cetrizin & Germany \\
\hline Chlordiazepoxide & Oral & Transillium & Argentina \\
\hline Dexamethasone & Dermal & Glymesason & Japan \\
\hline Dextromethorphan & Oral & Rynathisol & Europe \\
\hline $\begin{array}{l}\text { Diphenhydramin and } \\
\text { chlortheophyllin }\end{array}$ & Oral & Stada-Travel & Europe \\
\hline $\begin{array}{l}\text { Ethinylestradiol and } \\
\text { drospirenone }\end{array}$ & Oral & Yaz & Europe, United States \\
\hline Iodine & Topical & Mena-Gargle & Japan \\
\hline Meloxicam & Oral and Rectal & Mobitil & Egypt \\
\hline Nicotine & Sublingual & Nicorette & Europe \\
\hline Nimesulide & Oral & Nimedex, Mesulid & Europe \\
\hline Nitroglycerin & Sublingual & Nitropen & Japan \\
\hline Omeprazole & Oral & Omebeta & Europe \\
\hline Dinoprostone (PGE2) & Sublingual & Prostarmon E & Japan \\
\hline Piroxicam & Oral & Brexin & Europe \\
\hline Tiaprofenic acid & Oral & Surgamyl & Europe \\
\hline Cisapride & Rectal & Propulsid & Europe \\
\hline Hydrocortisone & Buccal & Dexocort & Europe \\
\hline Indomethacin & Eye drops & Indocid & Europe \\
\hline Itraconazole & Oral, intravenous & Sporanox & Europe, United States \\
\hline Mitomycin & Intravenous & Mitozytrex & United States \\
\hline Randomly methylated $\beta-C D$ & & & \\
\hline $17 \beta$-Oestradiol & Nasal spray & Aerodiol & Europe \\
\hline Chloramphenicol & Eye drops & Clorocil & Europe \\
\hline Sulphobutylether $\beta-C D$ & & & \\
\hline Aripiprazole & Intramuscular & Abilify & United States \\
\hline Maropitant & Intravenous & Cerenia & United States \\
\hline Voriconazole & Intravenous & Vfend & Europe, United States \\
\hline Ziprasidone maleate & Intramuscular & Geodon, Zeldox & Europe, United States \\
\hline Diclofenac sodium & Eye drops & Voltaren & Europe \\
\hline
\end{tabular}




\section{CHAPTER 2. SOLID DISPERSION REVIEW}

This chapter is intended to combine recent literature on solid dispersion technology for solubility enhancement with various preparation methods, evaluation parameters and commonly used polymer carriers. Solubility behavior is one of the most challenging aspects for drug commercialization or one of the main reasons for a drug that does not reach to its full potential. Currently, nearly $60 \%$ of new chemical entities possess solubility problems, whereas practically no drug products with less than $10 \mu \mathrm{g} / \mathrm{ml}$ solubility in 70's or 80's. Various techniques have been applied to enhance drug solubility such as the use of co-solvents, particle size reduction, lipid based drug delivery systems, nanosuspensions, use of surfactants, salt formation, cyclodextrin complexes and solid dispersions etc. In this chapter, several drug products using solid dispersion techniques are also reviewed.

\subsection{Definition and History of Solid Dispersion}

Chiou and Riegelman have defined solid dispersions in 1971 as "a dispersion of one or more active ingredients in an inert carrier at the solid state, prepared by the melting, the solvent or the melting solvent method" [42]. A solid dispersion consists of at least two components: hydrophilic matrix and hydrophobic drug. The matrix can be either crystalline or amorphous in structure. In 1971, solid dispersion was summarized as the following 6 types [42]: group 1, simple eutectic mixtures; group 2, solid solutions: a solid solute dissolved in a solid solvent, two components crystallize together in a homogeneous one-phase or more than one phase system; group 3, glass solutions and glass suspensions, the solute dissolved or dispersed in the glassy solvent; group 4, amorphous drug was dispersed in crystalline carrier; group 5, complex formation between the drug and the carrier; group 6, any combination among groups 1-5. From the point of physical state of drug in solid dispersion, drug was crystalline in simple eutectic mixture; in glass solutions, the drug and the carrier were both amorphous form. However, over the years, new formulation techniques for poorly soluble drugs made the line of classification less clear. The nanosuspension can be absorbed in solid absorbents to make nanoparticles dispersed and stabilized in solid matrices. Cocrystals can be classified into solid solutions and in lipid formulations or self-emulsifying drug delivery systems, drug can also be dispersed or dissolved in solid matrices [43]. The schematic diagram of the classification system and the new techniques overlap with it is shown in Figure 2-1. Nowadays, most solid dispersions are glass solutions or glass suspensions in which a hydrophilic polymer with high glass transition temperature was selected as carrier material and the drug can be molecularly dispersed or precipitated as amorphous form in the carrier [44]. The crystallization process can be prevented by the viscous medium. 


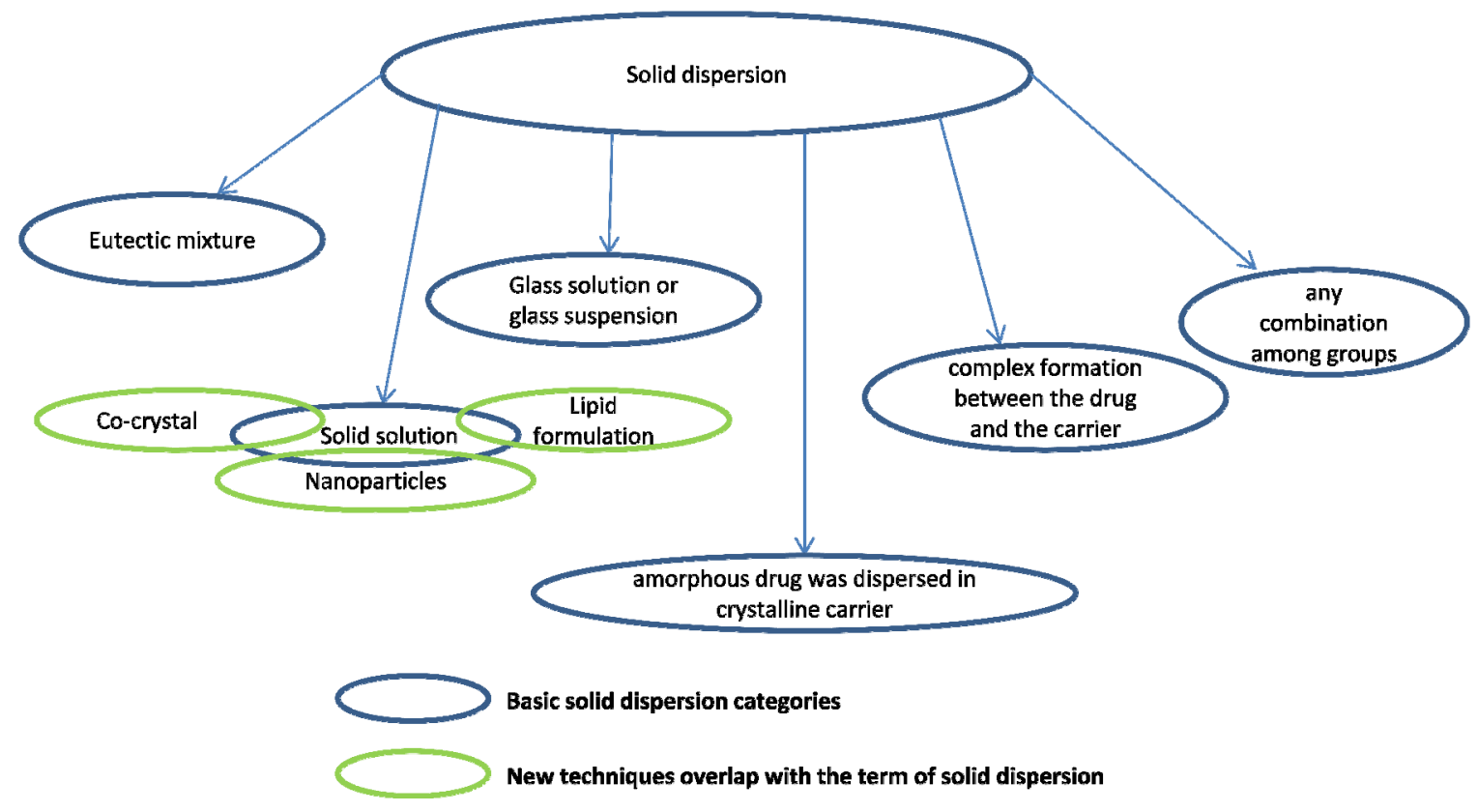

Figure 2-1. Scheme of solid dispersion classification system and new techniques.

\subsection{Dissolution Rate or Solubility Enhancement Mechanism from Solid Dispersion}

According to the preparation method, a number of strategies can be adapted to increase solubilization of poor water soluble drug and its dissolution rate in dosage form.

\subsubsection{Enhanced wettability}

A hydrophilic material as solid dispersion carrier can be utilized drug dissolution enhancement. The fast hydration of the surface of one phase solid dispersion system allows water to easily contact with the drug and the drug quickly release from dosage form [45]. Carriers with surface activity, such as surfactants can significant decrease the interfacial tension between dissolution medium and surface of dosage form, which also results in enhanced dissolution rate. The drug loading or the ratio between drug and carrier has a strong effect in the wettability of solid dispersions. Generally, a low drug loading or a high amount of polymer used in solid dispersion demonstrated better wettability $[46,47]$.

\subsubsection{Particle size reduction}

In a melt-quench method, drug and carrier are melted together and then quenched to form hard clump as one phase system. In the solvent evaporation method, drug and carrier are dissolved in common solvent and then evaporated rapidly. Solid dispersion 
clumps or powders left for further processing. In either of the two preparation methods, the drug is dispersed in the carrier at a molecular level. No distinguished drug particles can be observed in the solid dispersion. When drug releases from solid dispersion in aqueous media, the increased specific surface area of drug can be help to form colloid solution resulting in higher dissolution rate [43].

\subsubsection{Reduced aggregation and agglomeration}

Particle size reduction increases surface area of particles, thus it can improve the drug dissolution rate. However, for the pure fine particles $(0.1 \mu \mathrm{m})$, aggregation and agglomeration may commonly occur. Since the specific area of fine particles increases tremendously, the Van der waal's attraction and electrostatic charge among particles increases to cause particles to agglomerate and aggregate. When those pure fine particles meet with dissolution media, their poor water wettability properties made them dissolve slower than that of more coarse particles. In solid dispersions, this problem is overcome. The hydrophobic drug particle is surrounded by a matrix which prevented the hydrophobic attraction efficiently thus reduced the agglomeration or aggregation of particles [42]. The dissolution rate of a solid dispersion has been enhanced by the aid of solid dispersion hydrophilic carrier material. Various hydrophilic polymers form solid dispersion with poor water soluble compound demonstrated this point $[48,49]$.

\subsubsection{Amorphous state of drug}

Compared to its crystalline counterpart, the amorphous form of drug does not have a defined structure, and lower intramolecular or intermolecular attraction. Thus no energy is required to break crystal lattice in the amorphous state during dissolution. These properties allow the system to be easily hydrated and dissolved in water. The higher solubility promotes a fast dissolution rate $[50,51]$. The maintenance of supersaturation state of drug in dissolution media or GI tract is the major reason to promote a fast dissolution rate to achieve better bioavailability. The amount of polymer used in solid dispersion affects the drug supersaturation state significantly, the more polymer used, the higher degree of supersaturation [52]. Thus, using the advantage of amorphous form, keeping it stable and preventing its recrystallization has been a critical issue in formulation development. Selection of the carrier polymer, its ratio to drug, as well as preparation method play important role in a successful solid dispersion dosage form. More and more research demonstrated that the major mechanism of solid dispersion was the amorphous state of drug.

\subsection{Preparation Methods}

Traditionally, three basic methods have been used to prepare solid dispersion which is melting or fusion, solvent evaporation, and melting combined solvent evaporation method [42]. From the first solid dispersion formulation: sulfathiazole-urea 
solid dispersion published by Sekiguchi [43] in 1960s' till now, the research of solid dispersion is constantly expanding. The concentrated research has encouraged the emergence of many novel methods to prepare solid dispersion in lab scale, pilot and scale-up to manufacture size. The hot melt extrusion approach and spray drying technique have made solid dispersion dosage form commercialization possible. The two techniques have been the major processes in solid dispersion drug product manufacture.

\subsubsection{Hot melt extrusion}

The principle is co-melting or co-fusion: drug and carrier were heated together until melting to a clear liquid, and then quenched the melt to get solid dispersion. This method was first described by Sekiguchi and Ovi [53]. They prepared solid dispersion by melting the sulfathiazole-urea mixture, cooling the melt in an ice bath, and then the hard cooled melt was pulverized into powder. Nowadays, many solid dispersion experiments conducted in laboratories still use this method. Hot melt extrusion is an industrial applicable variation of the melting or fusion method. A specific machine has been designed and applied for this purpose. Hot melt extrusion equipment is commercially available from laboratory scale or manual operation to manufacturing scale or automatic operation. The procedures described below for the preparation of solid dispersion using hot melt extrusion. A powder blend of drug and polymer carrier is introduced into a heated barrel via hopper. A single-screw or twin-screw is installed in the barrel where the powder blend is intensively mixed, heated and moved to a shaping part which shapes the soft materials as granules, small cylinders, or films via various dies. Then the outcome of hot-melt extrusion will be further processed into a capsule or tablet. The simple flow chart of this procedure is shown in Figure 2-2. The feeding hopper, barrel with screw inside, die system and engine are the basic components consist of hot melt extrusion. A schematic diagram of hot melt extrusion is shown in Figure 2-3, modified according to [54].

In the heating barrel, a single screw or twin screw is installed to move the mixing melt. Compared to the single screw extruder, the twin screw extruder has some advantages: material can be fed easier and quicker; it has high kneading potential and material dispensing capacity; it is more efficient heating and mixing make the chance of material overheating less and shortens the operation time. Two rotation modes are designed for twin screw extruder. The two crews can co-rotate or counter-rotate. The commonly used rotate mode is co-rotating which can operate under high screw speed and more flexible design in screws. For counter-rotating extruder, a low screw speed is preferred since high screw speed will introduce a high pressure and high shear force

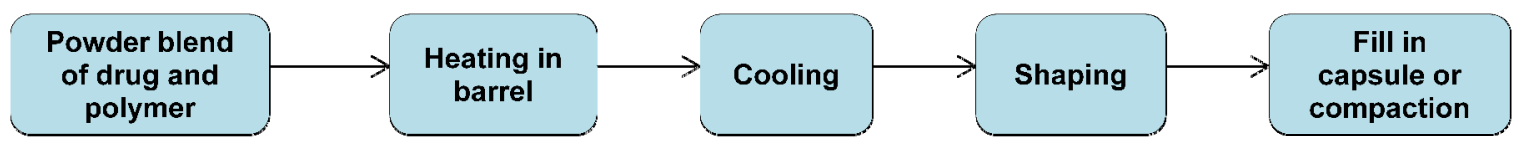

Figure 2-2. Schematic flow chart of hot melt extrusion process. 


\section{Hopper}

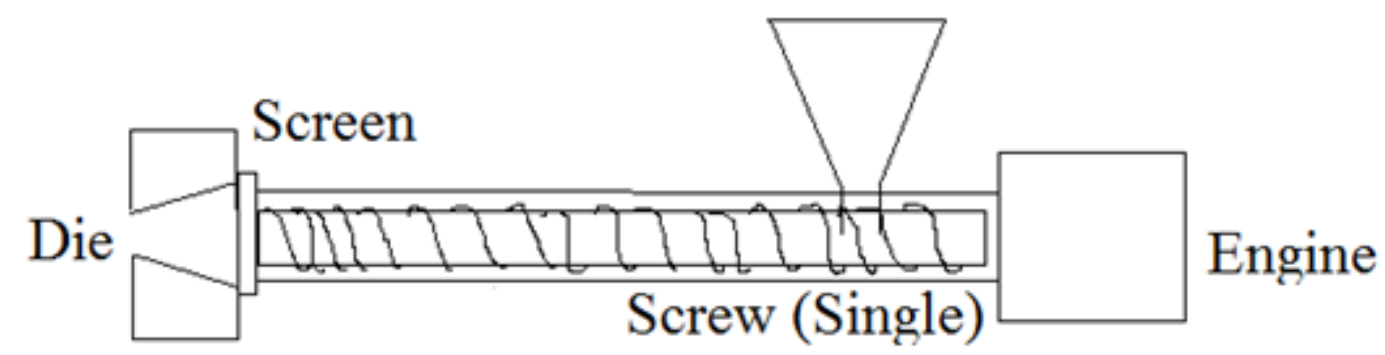

Figure 2-3. Schematic diagram of components of a single screw extrusion.

between screws which has high potential to damage the parts. High speed can also entrap air which cause defect in final extrudates [55]. However, the low cost and mechanically simplicity of the single screw extruder make it still one of the selections for users.

Several parameters in the hot melt extrusion procedure need to be considered and optimized for a specific experimental process. Those parameters include the barrel temperature, material feeding rate and screw speed. The melt pressure is decided by other parameters. The barrel temperature is decided by the melting point of the drug and the glass transition temperature of polymer and it is accurately controlled by instrument in a range of $30^{\circ} \mathrm{C}$ to $250^{\circ} \mathrm{C}$.

Besides the application in pharmaceutical industry, hot-melt extrusion is widely used in plastic and rubber industry to manufacture pipes, hosed insulated wires and cables, etc. In pet food industry, extrusion has been commonly used to produce palletized feeds for animals [54]. It has been used in food industry for pasta or ground meat production since 1930's [56].

Solid dispersion prepared using hot-melt extrusion method is used not only in oral dosage form development to help in solubilization of poor water soluble drugs but also used in transdermal delivery system, implants and sustained release drug delivery system. Several marketed solid dispersion drug products are listed in Table 2-1.

Besides marketed drug products, many research papers about solid dispersion study using hot melt extrusion technique have been published. The model drugs selected in these papers are generally BCS class II or IV compounds. Only the latest literature will be reviewed below since many papers have been covered in former review articles $[42,43,46,50]$.

Andrews et al investigated solid dispersion of bicalutamide and PVP prepared by hot melt extrusion technology. A single Tg in DSC, no sharp peak diffraction pattern of PXRD and enhanced dissolution rate indicated amorphous bicalutamide and PVP solid 
Table 2-1. Examples of commercial solid dispersions.

\begin{tabular}{ccccccc}
\hline Number & $\begin{array}{c}\text { Drug } \\
\text { product }\end{array}$ & Company & $\begin{array}{c}\text { FDA } \\
\text { approval }\end{array}$ & Technology & Drug & Carrier \\
\hline 1 & Gris-PEG & Pedinol & 1975 & Melt process & Griseofulvin & PEG 6000 \\
2 & Cesamet & Eli Lilly & 1985 & Melt extrusion & Nabilone & PVP \\
3 & Prograf & Fujisawa & 1994 & Unkown & Tacrolimus & HPMC \\
4 & Sporanox & J\&J & 1996 & Spray dry onto & Itraconazole & HPMC \\
5 & Certican & Novartis & 2004 & Unkown & Everolimus & HPMC \\
6 & Kaletra & Abbott & 2005 & Melt-extrusion & Lopinavir, & PVPVA \\
7 & Isoptin SR-E & Abbott & 2005 & Hot melt extrusion & Verapamil & HPC/HPM \\
8 & Intelence & Tibotec & 2009 & Spray drying & Etravirin & HPMC \\
9 & Nivadil & Fujisawa & Not yet & Unkown & Nivaldipine & HPMC \\
10 & Incivek & Vertex & 2011 & Spray drying & Telaprevir & HPMCAS \\
\hline
\end{tabular}


dispersion formed. Moreover, stability test has been done at 20 degrees $\mathrm{C}, 40 \% \mathrm{RH}$ and 20 degrees $\mathrm{C}, 65 \% \mathrm{RH}$ for 12 months, no drug crystallized was observed at $40 \% \mathrm{RH}$, whereas drug crystallized at higher humidity $(65 \% \mathrm{RH})$. However, drug recrystallization after storage did not change the dissolution profile of the solid dispersion [57]. Two methods: hot melt extrusion and cogrinding have been applied to prepare ginsenosides solid dispersion. In the dissolution test, the solid dispersion prepared by hot melt method is better than that of solid dispersion prepared by co-grinding method; however, cogrinding method showed better bioavailability than that of hot melt method [58].

Feng $\mathrm{J}$ et al investigated the hot melt solid dispersion of bifendate with three polymers: Plasdone ${ }^{\circledR}$ S-630, Eudragit ${ }^{\circledR}$ EPO and Kollidon ${ }^{\circledR}$ VA 64 . DSC and XRPD indicated that the drug was in amorphous form and dispersed molecularly in the three solid dispersions. The drug release from solid dispersions was in the order of bifendateKollidon ${ }^{\circledR}$ VA $64>$ bifendate-Plasdone ${ }^{\circledR}$ S- $630>$ bifendate-Eudragit ${ }^{\circledR}$ EPO, while the relative bioavailability tested in beagle dog was in the order of bifendate-Kollidon ${ }^{\circledR}$ VA $64>$ bifendate-Eudragit $\AA$ EPO $>$ bifendate-Plasdone ${ }^{\circledR}$ S-630. The relative bioavailability of bifendate-Plasdone ${ }^{\circledR}$ S-630 solid dispersion was lower than that of commercial pills [59].

Jijun $\mathrm{F}$ et al using hot-melt extrusion prepared a solid dispersion of nimodipine and two polymer mixture: Eudragit EPO and Kollidon VA64. The dissolution result showed fast drug release from solid dispersion. PXRD and DSC results showed that drug was in amorphous form in solid dispersion. The accelerated stability (40 degrees $\mathrm{C}, \mathrm{RH}$ $75 \%$ ) of solid dispersion tablet was examined for 2 months. Drug dissolution rate was slightly decreased after 2 month storage even though no crystalline was observed by PXRD [60].

Another BCS class II drug: celecoxib has been selected as model drug to prepare solid dispersion using hot-melt extrusion with PVP and Eudragit 4155F. Drug concentration in dissolution media was significantly higher than its equilibrium solubility. But the mechanism for this phenomenon was different for the two solid dispersions. For celecoxib-PVP solid dispersion, the supersaturation state and amorphous form of drug can be kept in dissolution media by PVP; while for celecoxib-Eudragit $4155 \mathrm{~F}$ solid dispersion, the polymer also acted as solubilizer that increased the equilibrium solubility of drug [52].

Two polymers Eudragit E100 and PVP-VA have been selected to prepare solid dispersion using hot-melt extrusion for fenofibrate which is a BCS class II drug. DSC and $\mathrm{X}$-ray diffraction showed that the compound was in amorphous form in the two solid dispersions. The results of drug release in $0.1 \mathrm{M} \mathrm{HCl}$ and water showed that Eudragit E100 was better than PVPVA in accelerating drug release. The relative bioavailability of fenofibrate-Eudragit E100 1:4 (w/w) solid dispersion was 177.1\% compared to micronized Lipanthyl capsule when tested in beagle dog [61].

Solid dispersion of nimodipine with HPMC, PVPVA and Eudragit EPO has been prepared using hot-melt extrusion. The dissolution results indicated that three polymers 
are suitable carriers to enhance the in vitro dissolution rate of nimodipine in $\mathrm{pH} 4.5$ medium. XRPD and DSC data showed that drug was in amorphous form in hot-melt extrudates. Nimodipine was miscible with HPMC when drug loading was $10 \%$ and phase separation existed when drug loading was increased to $30 \%$ and $50 \%$. The weakening and shift of the N-H stretching vibration of the secondary amine groups of nimodipine as determined by FT-IR showed hydrogen bonding between the drug and polymers in the solid dispersion [62]. The bioavailability study was conducted of the three solid dispersions in beagle dogs. The HPMC and PVP/VA dispersions exhibited much lower bioavailability compared to that of Eudragit EPO solid dispersion [63].

Hot-melt extrusion has attracted considerable interest within the pharmaceutical industry. However, how to identify the nature of the drug dispersion within the polymer remains a challenge. The following summary of three articles simply described the major techniques using for solid dispersion characterization.

Sheng Qi et al developed a combined thermal, imaging and spectroscopic approach for the identification and characterization of the drug and polymer structure. DSC run at various scan rates, MDSC, microthermal analysis (mu-TA) and ATR-FTIR were used to characterize the solid dispersions prepared by hot-melt extrusion containing $10 \%$ and 20\% paracetamol in Eudragit E. Dispersion of the drug in the polymer on a molecular basis was indicated by observed single Tg. However, DSC and ATR-FTIR also indicated that the drug was present in both amorphous and crystalline forms simultaneously. The ATR-FTIR also tested that the crystalline of drug was major in the center of extrudate. This study indicated that a single technique may not provide enough required information for fully understanding the complex system [64].

The physical and viscoelastic properties of the hot-melt extrusion prepared solid dispersions: indomethacin (INM) with Eudragit EPO (EPO), PVP-VA, PVPK30, and poloxamer 188 (P188) have been investigated by Chokshi RJ et al., A single T(g) was observed from the thermal analysis studies indicating good miscibility of drug and polymer for INM/EPO, INM/PVPVA, and INM/PVPK30 systems. In the case of INM/P188 solid dispersion, two melting endotherms were observed indicating partial miscibility of drug in polymer. Zero rate viscosity (eta(o)) and activation energy (E(a)) was determined for the various systems. The eta(o) of INM/EPO, INM/PVPVA, and INM/PVPK30 systems were found to be significantly lower as compared to pure polymer, indicating good miscibility of drug and polymers. INM/P188 mixtures showed a higher eta(o) compared to pure polymer indicating partial miscibility of drug and polymer. With respect to E(a), INM/EPO mixture showed an increased E(a) according to drug concentration increase, suggesting antiplasticization effect of the drug. The mixtures of INM/PVP-VA showed a decrease in the E(a) with the increasing drug concentration suggesting a plasticization effect of the drug. Considering these findings, a model was developed to correlate the zero rate viscosity to the motor load for hot-melt extrusion process [65].

The state of solid dispersions dissolved in dissolution media was investigated by Tho I et al. Ritonacir-PVPVA-Span 20 solid dispersion was prepared using hot-melt 
extrusion. Melt extrudates with and without ritonavir were studied. Nano/microdispersions formed for both drug containing and placebo system when the melt extrudate dissolved in dissolution media. The Nano/micro-dispersions are sensitive to anions and may form association, aggregation or flocculation overtime. The conclusion of such study was that the drug delivery system itself plays a key role for the formation of the observed particles [66].

Application of hot-melt extrusion method for thermo sensitive drug remains a challenge due to exposure to high temperatures. Three cellulose based polymers with relative low Tg; HPMC 3cps, HPMCP and HPMCAS were selected to prepare solid dispersions with a poorly water soluble drug, NVS981, which degrades upon melting at $165^{\circ} \mathrm{C}$. Two different drug loads of 20 and $50 \%(\mathrm{w} / \mathrm{w})$ were studied in each polymer system. Solid dispersions with $50 \%(\mathrm{w} / \mathrm{w})$ drug loading were easier to process in the melt extruder compared to that of $20 \%(\mathrm{w} / \mathrm{w})$ drug loading. High drug loading introduced better plasticizing effect to the system. Among the three polymers, HPMCAS was found to be the best one as it was easily processed and provided stable solid dispersions after 3 months storage at accelerated stability conditions with enhanced dissolution [67].

Hot melt extrusion (HME) and KinetiSol Dispersing (KSD) which is a new fusion-based process developed to rapidly form solid dispersions by imparting high shear and frictional forces were utilized to prepare solid dispersions of a BCS class II compound ROA with Eudragit L100-55 and HPMCAS. The compound was chemically thermal sensitive and unstable in acidic $\mathrm{pH}$. Since the amorphous form can't be obtained by hot-melt extrusion, the KSD process was investigated to reduce the decomposition of ROA while rendering compositions amorphous. The results of the study demonstrated that KSD is an effective method of forming dissolution-enhanced amorphous solids [68].

A novel hot melt extrusion strategy has been proposed to handle thermal sensitive drugs. Lakshman JP et al combined spray drying and hot-melt extrusion together to prepare solid dispersion. The high melting point drug was first processed to get amorphous form using a solvent evaporation method by spray drying technique. Polymer and amorphous drug mixture were further processed using a hot-melt extrusion. This process bypassed the exposure of thermal sensitive drug to high temperature. In the hotmelt extrusion process, the drug substance itself acted as the plasticizer for the polymer. Homogeneous dispersion in the matrix and subsequent enhanced dissolution of the drug were further improved by adding surfactant in the system. This novel system showed higher bioavailability than formulations containing the crystalline API. In an accelerated stability test, no crystalline drug was observed [69].

Although many successful commercial products of hot melt extrusion solid dispersion have shown the distinguished benefit of this technique, a critical prerequisite to the manufacturing process which is the miscibility of the drug and its carrier polymer is still a limitation factor for hot melt extrusion application. The drug has to be dissolved in the carrier polymer in the molten form, when the melt is cooling down, the drug will be dispersed in the carrier at molecular level. Another limitation to the hot melt method is the thermostability of drug and polymer. Lower melting point of the drug-carrier 
system is preferred. Generally, drug is more stable when it was treated at lower temperature. If high temperature is needed in the process, drug and carrier have to be thermal stable and do not decompose or evaporate. Many drug candidates are thermally unstable; they will be decomposed upon high temperature or the temperature near their melting point. In this case, an alternative method, solvent evaporation method has been studied and applied to make solid dispersion [70]. The major and commercial process is spray drying. Several commercial solid dispersion drug products are using this technique which has been listed in Table 2-1. The principle and technical aspects will be discussed in detail below.

\subsubsection{Spray drying}

As an alternative method for hot melt extrusion, solvent evaporation method is also commonly used in solid dispersion preparation. Spray drying technique has been widely used in food industry for milk powder production, cartridge manufacture, and pesticide production. In the pharmaceutical industry, it is poplar in pharmaceutical excipient or co-processed excipient manufacture.

The basic steps for preparing solid dispersion using spray drying include: 1. Dissolving the API, polymer carrier and any other desired adjuvants in a common solvent; 2 . Atomising the liquid into fine droplets; 3 . Rapidly removing the solvent from the liquid droplets as they fall through the drying chamber; 4. Collecting the resulting powder; and 5. Further drying the powder to remove residual solvents. 6 . The final step was tableting or encapsulation. The Figure 2-4 showed this process.

A common spray drying instrument consists of sample feeding system, heating unit, atomization unit and sample collection unit. The machine made by different manufacturer has different design [71]. The schematic diagram of a basic spray drier is shown in Figure 2-5. The atomization unit is the key part of a spray dryer. Commonly, two fluid nozzle, pressure nozzles and rotating wheel atomizers are available to meet the requirement of laboratory scale, pilot scale and manufacture. For laboratory application, two-fluid nozzle is often used. Pressure (hydraulic) nozzles are often installed for manufacture because it is the most efficient way in atomization. Besides, rotary atomizers are also available for pilot scale.

When preparing solid dispersion using spray drying, a closed system is usually selected since the solvent can't be evaporated into air. The commonly used solvents include ethanol, acetone, ethyl acetate, tetrahydrofuran, methanol, dichloromethane,

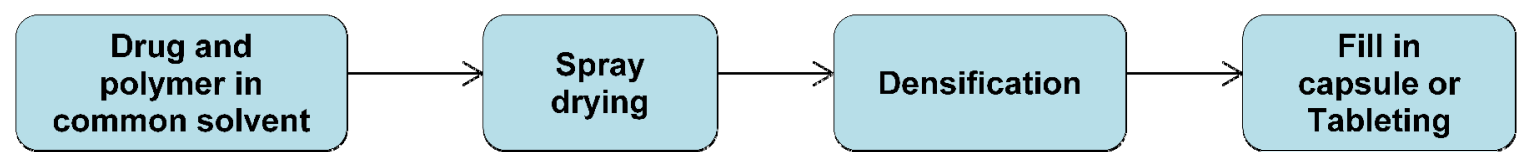

Figure 2-4. Scheme of solid dispersion preparation using spray drying technique. 


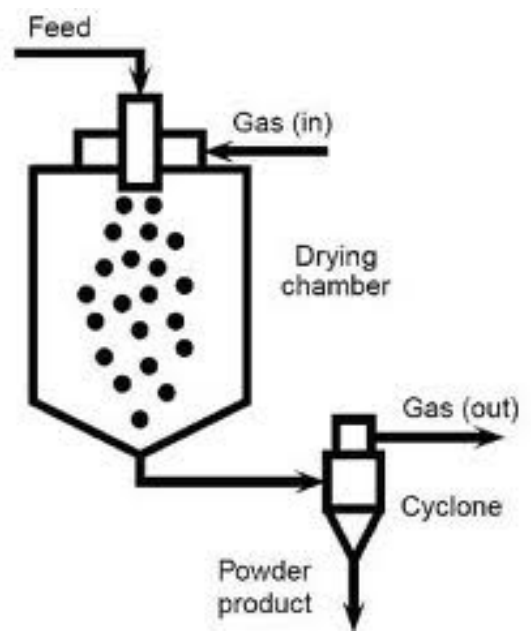

Figure 2-5. Components of spray dryer.

water and various combinations of these. Typical polymers include PVP, PVPVA, and HPMC, HPMCAS and other HPMC derivatives and acrylate copolymers. Addition of surfactants to stabilize the common solvent system is not uncommon [71]. The major advantages of spray drying technique are fast process time, the ability to work with thermal sensitive APIs, flexibility of process various solvent systems with various surfactants and polymers. After solvent is removed, the drug dispersed in the carrier matrix homogeneously at molecular level. In spray dried solid dispersions, particle size reduction, wettability enhancement and amorphous form acquisition of hydrophobic drug are obtained in one step. The consumption of organic solvents thus adding the costs and the followed requirement of residual removal are the major disadvantages of this technique.

The properties of spray dried solid dispersion, such as density, flowability and residual solvent can be controlled by adjusting process parameters, such as nozzle type selection, inlet temperature, outlet temperature and carrier gas volume. Particle size and distribution decide the flowability of spray dried powder which can be optimized by selecting nozzle. The two-fluid nozzle usually produces small particles, the size is around $2-30 \mu \mathrm{m}$, larger particles are produced by pressure nozzles; the higher inlet temperature can shorten the whole process time and the drying time, at the same time, residual solvent must be controlled at a low level. If the inlet temperature is too high, the outlet temperature will also be increased which may induce a sticking problem in the spray drying process when the outlet temperature is higher than the $\mathrm{Tg}$ of sample system or melting point of compound. However, low outlet temperature will keep more residual solvent in the spray dried powder which will be as plasticizer to reduce the Tg of solid dispersion system. Carrier gas provides heat energy to dry the sample, it can be air when sample has no organic solvent or can be inert gas, such as nitrogen gas when the sample contains organic solvent. The volume of carrier gas will affect the product yield. Generally, this parameter needs to be optimized according to different type of spray 
drying instrument [71]. In summary, optimization of spray drying process is an integrated process; decision for each parameter will affect other parameters. Considering the final property of solid dispersion powder, all parameters will be optimized and decided together. The commercialized drug product manufactured by spray drying method was shown in Table 2-1. The articles of spray dried solid dispersion published in recent years are reviewed as follows:

A ternary system: solid dispersions griseofulvin, PHPMA and PVP have been prepared using spray drying technique. Acetone and water or acetone and methanol were selected to dissolve drug and polymer. The solvent system affected the properties of solid dispersions significantly. Thermal analysis was done for the spray dried solid dispersion powders, high $\mathrm{Tg}$ was about 103 degrees $\mathrm{C}$ for the acetone/methanol system, while for acetone/water system, the Tg was 83 degrees $\mathrm{C}$. This is because the conformational variations of the polymers in solution prior to spray drying. The effect of drug and polymer concentration on the properties of solid dispersions was also studied. A lower relaxation rate was found to solid dispersions which spray dried from dilute drug-polymer solution [72].

Caron V et al investigated the sulfathiazole (STZ)/ PVP and sulfadimidine (SDM)/PVP solid dispersions preared by spray dying and co-milling method. Multiple analysis techniques have been applied to characterize the two solid dispersions. Both spray drying and co-milling method can produce homogeneous glass solutions for the two model drugs and the value of T(g) for a particular API/PVP ratio did not depend on the processing technique used. According to the Gordon-Taylor equation, the interaction between drugs and PVP are not stong which was indicated by the consistent results of measured Tg and predicted Tg and the variation of $\mathrm{T}(\mathrm{g})$ versus concentration of PVP was monotonic for all the systems. Amorphous SDM can be obtained on spray drying but not amorphous STZ. Flory-Huggins model was applied to calculate the miscibility of two APIs and PVP. The results showed that the two APIs have similar miscibility with PVP. Intrinsic dissolution studies in aqueous media revealed that dissolution rates of glass solutions were the fastest no matter the preparation method. A conclusion was made that co-milling can significantly enhance the dissolution of some poorly soluble drugs to a similar magnitude as co-spray dried systems [73].

A review article covered how the particle formed by spray drying technique from theory to practice. Some application examples in pharmaceutical industry -- low density particles, composite particles, microencapsulation, and glass stabilization -- are discussed, with specific emphasis on the underlying particle formation mechanisms and design concepts [74].

An application of poly(2-hydroxyethyl methacrylate) (PHEMA) hydrogel in solid dispersion has been studied. Three drugs: diclofenac sodium, piroxicam and naproxen are selected as model drugs. XRD, DSC and FTIR are used to characterize these solid dispersions and stability under various conditions. The results show that as high as 30\% drug loading is acceptable, above that will induce amorphous to crystalline transition. During storage, high $\mathrm{RH} \%$ will increase the recrystallization rate duing storage 
for any drug loading. The FTIR result indicates that hydrogen bonding presents among drug and polymer. Dissolution rate of the three model drugs in solid dispersion increased compare to their physical mixture. A conclusion has been made that the PHEMA can be used as potential solid dispersion carrier to enhance drug dissolution rate and futher bioavailability [75].

Solid dispersion piroxicam -PVP prepared by spray drying and supercritical CO2 fluid method has been studied by Ke Wu et al. DSC, FTIR and XRPD were applied to characterize properties of solid dispersion. Multiple techniques showed amorphous form was presnt in the binary system with $20 \%$ drug loading. The dissolution rate of piroxicam in solid dispersion prepared by supercritical $\mathrm{CO} 2$ fluid increased significantly compared to that of API itself. From the investigation, the author drew the conclusion that PCA (supercritical fluid $\mathrm{CO} 2$ ) processing could be an effective alternative method to prepare solid dispersion [76].

In the early stage of solid dispersion research, 1970s' to 1980s', PEG and PVP are the most common polar polymers. After that, semi-synthesized polymers, such as HPMC or its derivatives were used and studied as solid dispersion carriers. Besides PEG and PVP, HPMC became the third type of polymer that was applied in commercial drug products. Bend research Inc. recently published an article reviewed more than one hundred compounds that form solid dispersion with hydroxypropyl methylcellulose acetate succinate (HPMCAS) by spray drying method. These spray dried dispersions (SDDs) kept supersaturation and amorphous drug/polymer colloids in in vitro dissolution determinations and were found to have large bioavailability increases in vivo. At the same time, those compounds can remain amorphous form during storage. This large scale solid dispersion test proved that the spray drying technique and HPMCAS is a successful combination for solid dispersion preparation [77].

\subsubsection{Supercritical fluid extrusion}

Besides hot-melt extrusion and spray drying method, supercritical fluid has been also introduced as a solid dispersion preparation method. Supercritical fluid is neither gas nor liquid. However, it has the properties of gas and liquid at the same time. Under certain temperature and pressure, gas can form supercritical fluid which has the high density and low viscosity. The special fluid has the solvent capacity to extract constitutes from plants according to the polarity. Upon the adjustment of pressure, the supercritical fluid will extract material from low polarity to high polarity and then enter the separation unit, where supercritical fluid is separated with the extraction and then go to collection unit for recycle. Carbon dioxide ( $\mathrm{CO} 2)$ is the most commonly used media in supercritical fluid technique. In solid dispersion preparation, compound and polymer will be first dissolved in common solvent, then the sample is treated by $\mathrm{CO} 2$ supercritical fluid, solvent will be extracted and removed by $\mathrm{CO} 2$ fluid and the solid dispersion powder is separated and precipitated from solvent. The common solvent and $\mathrm{CO} 2$ will be further separated for recycle. The advantage of $\mathrm{CO} 2$ supercritical fluid method is its low cost and 
environmental friendly, the solvent residual in solid dispersion powder is less than that of spray drying method.

Rodier et al prepared eflucimibe- $\gamma$-cyclodextrin co-crystal solid dispersion using supercritical fluid extraction method. Both the solubility and the dissolution rate were improved. Eflucimibe and cyclodextrin have been dissolved in DMSO and then treated with supercritical carbon dioxide, solvent was removed. Then the co-crystal was kept under supercritical condition for several hours, then the solid dispersion was treated a second time by supercritical carbon dioxide to remove residual solvent. The three step operation process made efludimibe homogeneously dispersed in $\gamma$-cyclodextrin. The properties of solid dispersion are not sensitive to the operation parameters [78].

Won et al. have prepared felodepine solid dispersion using conventional solvent evaporation method, CSE and supercritical anti-solvent method, SAS. The carrier includes HPMC and surfactant. Felodepine is in amorphous form no matter the preparation method. The SAS method is better than CSE method after comparing the properties of the solid dispersions. The solid dispersion prepared by SAS method has smaller particle size, better flowability, less residual solvent, higher solubility and faster dissolution rate [79].

\subsubsection{Other methods}

Co-grinding and electrostatic spinning method are also used to prepare solid dispersion $[80,81]$.

\subsection{Characterization Techniques}

Many techniques are available to contribute information for explanation of the physicochemical phenomenon of solid dispersions. Generally, a combination of two or

more techniques is required to characterize a solid dispersion system. The advantages and disadvantages of each technique are briefly discussed below.

\subsubsection{Powder X-ray diffraction (XRPD)}

XRPD is applied to determine whether crystal or amorphous form exist in solid dispersion, it can also quantify crystalline degree if the crystalline standard curve can be made [82]. X-rays are considered radiation in the electromagnetic radiation spectrum, the wavelength of X-rays is around $0.01 \mathrm{~nm}$ to $10 \mathrm{~nm}$. Crystalline material has a characteristic atomic structure and it will diffract X-rays in a unique pattern. The amorphous form generally is indicated by a wide and flat peak, basically, no sharp peak appears in amorphous material X-ray spectrum. Based on the physical state of various materials, XRPD can also be used in measurement and prediction of crystal size and shape, protein structure characterization, etc. 
XRPD is a straightforward method to distinguish a crystalline from an amorphous system. Its sensitivity is around 5\%. In solid dispersion characterization, it is often combined with other techniques to characterize the phase separation, crystalline degree even the size of crystal. When determining the crystalline degree, a series standard sample needs to be prepared: 5 to 7 crystal concentration points are needed to construct a wide range standard curve with a determined limit of quantitation. Using a calibration curve to quantify the crystalline content is similar to that of other chemical quantify methods, such as HPLC.

Anthony Severdia et al used XRPD to determine the content of crystalline form I in crystalline form II. They mixed form I and II at different ratio and then constructed calibration curve using peak area or peak height. When they used peak area to construct standard curve, the R2 was better than that of peak height, but the difference was not large. The author also quantified the content of form I in form II using an NIR method. Basically, both XRPD and NIR methods can be used in crystalline quantitive analysis, but when the crystal content was lower than $5 \%$, the accuracy of measurement will be a problem [83].

\subsubsection{Thermal analysis methods}

Thermal analysis tools are often used in solid dispersion characterization. Differential scanning calorimetry, DSC and thermal gravimetric analysis, TGA are most commonly used. DSC can measure many thermal transition phenomena including melting point, glass transition point, or recrystallization transition, etc. These measurements provide qualitative and quantitative information that about physical and chemical changes that involve endothermic or exothermic processes or change in heat capacity. Modulated temperature DSC is derived from conventional DSC. The temperature is continually increasing in sinusoidal modulation (oscillation) way which is different from the linear fashion of traditional DSC. Thermal events respond differently to the oscillation heating rate. In MDSC, glass transition temperature can be easily observed and assigned. A disadvantage of MDSC is it is time consuming: one hour or longer time is needed for a typical MDSC data collection [84].

TGA measures mass change of a substance as a function of temperature while the substance is subjected to a controlled temperature program. If the substance contains a volatile fraction, the mass will be lost when the temperature is high enough. TGA is also combined with DSC to differentiate the true melting and decomposition. If a sample has the melting combined decomposition, DSC can only give an endothermic melting peak or exothermic peak. As for the exothermic peak, it is generally assigned as a decomposition peak; however, to confirm this assignment and remove the possibility of operation error, sample is further tested by TGA. The observed weight loss curve shown in the same temperature range on TGA will verify the results of DSC.

In nearly every published article related to solid dispersion, DSC has been applied as a routine tool to measure the glass transition temperature, melting point; to distinguish 
phase separation as well as to quantify the content of crystal in glass solution system. Generally, phase separation will be defined when two Tgs are found in DSC, however, this technique has its own limitation sometimes. The Tg of separated glass domain may not be measured by DSC if the separated domain is just about $30 \mathrm{~nm}$. Newman et al using XRPD coupled with computation of pair distribution functions (PDF) technique successfully found a $30 \mathrm{~nm}$ phase separation domain in indomethacin-PVP binary glass solution system [85].

\subsubsection{Fourier IR}

Fourier transform infrared spectrum is a specific character for compound. Commonly, the spectrum of a compound before process and after process will be compared to see if there is difference that can be found in the spectrum profile. The spectrum of crystalline and amorphous state of a compound usually reflects differences in FTIR. Based on this point, FTIR is applied together with other techniques to characterize solid dispersions. The advantages of this technique include the small sample size (about several micrograms), quick test time (several minutes) and relatively inexpensive and easy to operate.

FTIR was used to study the effect of compression on miscibility of naproxen-PVP K25 solid dispersions. FTIR analysis showed the unchanged profile of drug in solid dispersion upon compression when drug loading was $20 \%$. While, when drug loading was increased to $30 \%$ and $40 \%$, phase separation was induced by compression pressure (565.05MPa). The altered IR spectral profile and two Tg in DSC confirmed this point together [86].

Qi et al applied Attenuated Reflection Fourier Transform IR (ATR-FTIR) combined thermal and imaging technique to identify the paracetamol-Eudragit ${ }^{\circledR} \mathrm{E}$ solid dispersion. Spectrum evidence obtained from FTIR indicated the presence of crystalline in $10 \%$ and $20 \%$ drug loading solid dispersion system and the major location of crystalline was in the center of the extrudate [64].

\subsubsection{Scanning differential microscopy (SEM)}

SEM technique is a more direct tool to investigate solid dispersions. The morphology of solid dispersion powders or extrudates can be easily observed visually. Sometimes, SEM can show if the sample is crystalline or if there is phase separation. However it is limited by the resolution. The very small crystalline may not be observed by SEM technique. 


\subsubsection{Dynamic vapor sorption (DVS)}

DVS is applied to determine the moisture sorption properties of pharmaceutical materials such as excipients, drug formulations and packaging films. These properties are recognized as critical factors in determining the storage, stability, processing and application performance of drug products $[87,88]$. Further, organic solvent and water can be selected to study solvate or hydrate formation [89]. It can also be used as the very sensitive method to qualitatively and quantitatively determine amorphous contents [90].

Lesley et al. apply DVS technique to determine the amorphous content in a micronized compound. Water and acetone are used to provide vapor pressure to construct the calibration curve. In the experiment, $100 \%$ amorphous and 100\% crystalline states are characterized by a variety of techniques. Water vapor does not induce recrystallization but acetone vapor can induce recrystallization. The lowest quantitative limit of this DVS method for amorphous content determination is $0.5 \%$. The measurement is in agreement with the results that are determined by DSC [90].

\subsubsection{Solid state NMR and 2D NMR}

Solid-state NMR (SSNMR) can provide detailed structural information about amorphous solid dispersions of pharmaceutical small molecules. In contrast with solution state NMR, solid-state NMR (ssNMR) does not require that the sample be soluble or form a crystal, and no specific NMR solvent consumed. Two dimensional NMR is usually combined with this technique in solid dispersion characterization.

In SSNMR, dipolar correlation, spin diffusion, and relaxation measurements can be separately applied or combined to characterize the structure of solid dispersions. Observation of spin diffusion effects using the 2D H1-C13 cross-polarization heteronuclear correlation (CP-HETCOR) can show glass solution formation. H1 doublequantum correlation experiments to probe interaction at molecular level between polymer and drug. H1-F19 CP-HETCOR serves a similar role as CP-HETCOR for fluorinated drug molecules. The sharpness degree of peak in NMR spectrum and difference to its amorphous or crystal control indicate if there is glass solution formation. Phase separation is detected using C13, F19, and Na23-detected H1 T1 experiments, dipolar correlation and spin diffusion effect are observed to prove the molecular interaction between drug and polymer. Two more examples: solid dispersion ternary system of voriconazole and telithromycin with PVP and SLS are analyzed to demonstrate the general applicability of the technique [91].

Lefort et al determines the amorphous content of trehalose in mixture of amorphous and crystal trehalose using SSNMR. The results of C13NMR method is compared to that of DSC technique. The good agreement of the two methods indicates that both are useful and accurate methods for compositional characterization of powders [92]. 


\subsection{Conclusion}

The review summarizes several aspects of solid dispersion: concept definition, background, commercial products, solubilization mechanisms, preparation methods, characterization techniques. Solid dispersion is shown to be a fruitful approach to increase the bioavailability of poorly soluble drugs because of increasing number of drug candidates with low solubility. In addition to binary solid dispersion systems, researchers also add surfactant or cyclodextrin in drug-polymer system to form ternary solid dispersion for better drug performance. It has been a common strategy for poorly soluble drug development. 


\section{CHAPTER 3. AQUEOUS SOLUBILITY, DISSOLUTION, PHYSICOCHEMICAL CHARACTERIZATION AND PHYSICAL STABILITY OF SOLID DISPERSIONS OF FUROSEMIDE AND OCTENYL SUCCINIC ANHYDRATE STARCH}

The purpose of the present study was to investigate the applicabability of a cold water soluble modified starch, octenyl succinic anhydrate starch (OSA starch) as solid dispersion carrier to increase the aqueous solubility and dissolution rate of poorly water soluble drug. Furosemide was selected as a model drug. The solid dispersion of furosemide with OSA starch was prepared using spray drying technique via various sample preparation methods: furosemide dissolved in ammonia solution (salt control), furosemide and OSA starch dissolved in ammonia solution (amorphous dispersion,) and furosemide dispersed in OSA starch solution (crystal dispersion). Physical mixtures (PM) were also prepared for comparison. Three ratios between drug and OSA starch: 1:1, 1:2.3 and 1:9 w/w were selected and tested. The $\mathrm{pH}$ of solid dispersions, aqueous solubility and dissolution (under $\mathrm{pH} 1.2$ and $\mathrm{pH}$ 5.8) of the drug in these solid dispersions were evaluated compared to drug physical mixtures. Furthermore, their physicochemical properties were investigated using SEM, FTIR, MDSC, XRPD and Dynamic vapor sorption (DVS). Physical stability tests were done under $40{ }^{\circ} \mathrm{C}$ and $40{ }^{\circ} \mathrm{C}$ with $75 \% \mathrm{RH}$ for 3 months, furosemide kept its amorphous form in all samples at $40^{\circ} \mathrm{C}$, while crystallization was found in samples treated under $40{ }^{\circ} \mathrm{C} 75 \% \mathrm{RH}$ conditions. The solubility improvement of samples was significant and in the order of: amorphous dispersion $>$ salt control $>$ crystal dispersion $=$ PM. Tablets were compressed for dissolution test. In phosphate buffer ( $\mathrm{pH}$ 5.8), drug released near completely in 20 minutes for all samples except PM (1:1 drug: polymer w/w). In simulated gastric fluid (SGF, pH 1.2), the drug dissolution was tested for one hour and the dissolution rate was as the rank of: amorphous dispersion $(1: 9 \mathrm{w} / \mathrm{w}$ drug: polymer $)>$ crystal dispersion $(1: 1>1: 2.3>1: 9 \mathrm{w} / \mathrm{w}$ drug: polymer $)>$ PM $(1: 9>1: 2.3 \mathrm{w} / \mathrm{w}$ drug: polymer $)>$ amorphous dispersion (1:2.3 w/w drug: polymer) $>$ PM (1:1 w/w drug: polymer) $>$ Lasix ${ }^{\circledR}>$ amorphous dispersion (1:1 w/w drug: polymer) $>$ salt control. At the 60 minute point, only amorphous dispersion (1:9 w/w drug: polymer) released $80 \%$ API and the highest amount of drug released was $65 \%$ for other samples. Using a high amount of OSA starch in amorphous dispersions to keep a supersaturation state of furosemide in acidic conditions was thought to be the principle factor for dissolution improvement. From the study, OSA starch can be applied as a solid dispersion carrier with controlled humidity. Both the preparation method and starch amount play critical role in developing a binary solid dispersion system for a poor water soluble drug.

\subsection{Introduction}

Solid dispersion is one of the drug solubilization techniques that is utilized to formulate poorly water-soluble drugs. It is a well-established method that has successfully brought several poorly soluble drugs to market, e.g. telaprevir, etravirin and verapamil, etc. The major preparation method includes melting and solvent-evaporation 
method [93]. Basically, drug and polymer mixture is heated until it melts and then quenched cool to get solid dispersions or dissolve drug and polymer in a common solvent and then spray drying the solvent to get solid dispersion. Moreover, another method: surface-attached solid dispersion has been developed to avoid using organic solvent. Drug is dispersed in polymer water solution and remains in crystal form with small particle size upon evaporation of water [94]. This solid dispersion could increase solubility and bioavailability of drug and at the same time, however not change its crystalline state. The major mechanisms of solid dispersion techniques contribute include one or more of the following: enhanced water wettability, particle size reduction, reduced aggregation and agglomeration and enabling creation of an amorphous state [42, 43].

OSA starch is made by esterification of already hydrolyzed and depolymerized starch and anhydrous octenylsuccinic acid under alkaline conditions [95]. OSA treated starches contain hydrophobic octenyl side chains which impart an emulsifying capability to the starches. Thus, the OSA starch bears an amphiphilic property that has been widely used for many years for flavor encapsulation and emulsifier for beverage emulsions. It has good cold water solubility, low viscosity of solution at high solid content, nonhygroscopic character, bland taste, non-reactivity and low cost. All these advantages make it a good spray drying carrier material [96].

When OSA starch is used in solid dispersions via spray drying technique, the hypothesis is that the drug dissolved in starch solution. When water evaporates via spray drying, the drug will be solidified together with starch and mostly the drug is in amorphous form because the very fast spray drying process (seconds) prevents drug crystallization. In the spray dried powder, the drug won't be distinguished from the starch matrix. In this system, starch is homogenously distributed in the matrix thus prevents drug crystalline growth. When drug is dispersed in starch solution, starch will encapsulate drug particles to complete the microencapsulation process. Micro-scale drug particles will be retained in the starch matrix. These properties will be helpful in enhancing drug solubility and dissolution rate.

Furosemide is a loop diuretic that acts on the kidney to remove excess water and electrolytes into urine, thus reducing the body fluids. Furosemide was used to treat hypertension, congestive heart failure and edema [97]. Furosemide is a BCS class IV drug. It is often selected as model drug to test new formulation techniques or as candidate for drug optimization because of its low and various bioavailabilities [97, 98]. Many oral formulations have been used in order to increase solubility of furosemide such as cyclodextrin complexes [99-101], dendrimer complexes [102], self micro-emulsifying drug delivery systems [103], mesoporous silicon microparticles [104], solid dispersion with various carriers [105-108] have been investigated.

The purpose of present study is to explore the applicabability of OSA starch as solid dispersion carrier in the pharmaceutical field. Since it is a good spray drying agent, spray drying technique is one of the major methods for solid dispersion preparation, and because of its amphiphilic property, OSA starch is theoretically considered as a solid dispersion carrier. Thus, experiments are conducted to evaluate the effect of OSA starch, 
preparation method on the solubility, crystalline and dissolution of model drug in solid dispersions. Solid dispersions containing furosemide were prepared as salt control, amorphous dispersions and crystal dispersions. Solubility and dissolution were determined for these solid dispersions. Multiple techniques have been applied to investigate their physicochemical properties and solubilization mechanisms. Accelerated stability test was conducted at $40{ }^{\circ} \mathrm{C}$, controlled humidity and $40{ }^{\circ} \mathrm{C}, 75 \% \mathrm{RH}$ for three months to fully evaluate the quality of solid dispersion.

\subsection{Material and Methods}

\subsubsection{Material}

Furosemide was generously donated by Teva Pharmaceuticals (Petah Tikva, Israel), OSA starch was provided by National Starch \& Chemical (Bridgewater, NJ). Ammonia aqueous solution was 29.4\%. All other chemicals were reagent grade and used as received without further processing.

\subsubsection{Preparation of furosemide-loaded solid dispersions}

A Büchi 290 mini spray dryer with $0.7 \mathrm{~mm}$ nozzle (Flawil, Switzerland) was used for the preparation of the furosemide-loaded solid dispersions. Since furosemide was weak acid with pKa 3.8, its solubility is $\mathrm{pH}$ dependent. Ammonia solution was used to dissolve certain amount of furosemide. All liquid samples were delivered to the nozzle at a flow rate of $5 \mathrm{~mL} / \mathrm{min}$ via a peristaltic pump and spray-dried at $120{ }^{\circ} \mathrm{C}$ inlet temperature and $65-70{ }^{\circ} \mathrm{C}$ outlet temperature. The Q-flow was $45-50 \mathrm{~mm}$ and the aspirator was set as $90-100 \%$.

\subsubsection{Salt control preparation}

A certain amount of furosemide $(2 \mathrm{~g})$ dissolved in $65 \mathrm{~mL}$ ammonia water: the molar ratios between furosemide and ammonia were 1:1.4 (I), 1:3.6 (II), 1:6.0 (III) and 1:17.9 (IV). The ratio 1:1.4 was the minimum molar ratio to dissolve furosemide in aqueous ammonia. Then these aqueous solutions were spray dried to get ammonia salt control samples. These salt solid dispersions were vacuum dried overnight to remove residual ammonia and then store in refrigerator for fully characterization. By aid of a basic environment that ammonia provided, furosemide and OSA starch can be easily dissolved in water at the same time. This is the critical step for solid dispersion preparation using solvent-evaporation method. 


\subsubsection{Solution solid dispersion preparation (Soln. SD)}

Three ratios $(1: 1,1: 2.3$ and 1:9 $\mathrm{w} / \mathrm{w})$ between furosemide and OSA starch were selected to prepare amorphous solid dispersions. Ammonia solution (29.4\%) $0.5 \mathrm{ml}$ was added to $35 \mathrm{ml}$ water, then $1 \mathrm{~g}$ furosemide was added into ammonia solution, then OSA starch $1 \mathrm{~g}, 2.3 \mathrm{~g}$ and $9 \mathrm{~g}$ were added to the furosemide ammonia solution separately. In all amorphous dispersion samples, the ratio between furosemide and ammonia was 1:1.4 ( $\mathrm{mol} / \mathrm{mol})$. After stirring and mixing well, these colloid solutions were spray dried to get powder. These solid dispersion powders were stored at refrigerator for characterization after drying in vacuum oven overnight.

\subsubsection{Suspension solid dispersion preparation (Susp. SD)}

OSA starch $1 \mathrm{~g}, 2.3 \mathrm{~g}$ and $9 \mathrm{~g}$ dissolved in $35 \mathrm{ml}$ water, and then $1 \mathrm{~g}$ furosemide was added into starch solution, less than 5\% PEG 400 was added into the liquid as dispersion stabilizer to prevent precipitation during spray drying process. Then the liquid was converted to powders after spray drying. Then the same process step was applied for the powder as former samples.

\subsubsection{Physical mixture (PM)}

OSA starch $1 \mathrm{~g}, 2.3 \mathrm{~g}$ and $9 \mathrm{~g}$ were mixing with same amount of furosemide using liquid-solids blender (type: LB-4455, The Patterson-Kelley Co, east Stroudsburg, PA.) for 3 min each. Then store in regenerator for further characterization.

\subsubsection{Aqueous solubility measurements}

Excess amounts of furosemide (about $10 \mathrm{mg}$ ) for salt control, amorphous dispersion, crystal dispersion powders and physical mixtures were added into triplicate 20 $\mathrm{ml} \mathrm{screw-capped} \mathrm{vial} \mathrm{that} \mathrm{contained} 10 \mathrm{ml} \mathrm{R.O}$. water ( $\mathrm{pH} \mathrm{6.2).} \mathrm{The} \mathrm{vials} \mathrm{were} \mathrm{vortexed}$ for 30 seconds at $1000 \mathrm{rpm}$, and then put on a shaker, shaking at room temperature (25 ${ }^{\circ} \mathrm{C} \pm 1{ }^{\circ} \mathrm{C}$ ) for $24 \mathrm{~h}$, centrifuged at $3000 \times \mathrm{g}$ for $10 \mathrm{~min}$ (Eppendorf, NY), filtered through a membrane filter $(0.22 \mu \mathrm{m})$ and then diluted to proper concentration for analysis by HPLC. The HPLC system was a HP 1100 system together with a Nova-Pak ${ }^{\circledR} \mathrm{C} 18$ Column (Waters, $4 \mu \mathrm{m}, 150 \mathrm{~mm}$ X $3.9 \mathrm{~mm}$, i.d.) and a UV Detector. The mobile phase was $10 \mathrm{mM}$ potassium phosphate monobasic and methanol $(60 / 40, \mathrm{v} / \mathrm{v}), \mathrm{pH}$ adjusted to 2.5 by phosphoric acid. The flow rate was $1 \mathrm{ml} / \mathrm{min}$, injection volume was $10 \mu 1$ and the wavelength was $276 \mathrm{~nm}$. 


\subsection{4 pH measurements}

After spray drying and vacuum oven drying, free ammonia should be removed from samples because of the low partial pressure of ammonia. For API, salt control, solid dispersion and physical mixture, a $1 \%$ dispersion $(\mathrm{w} / \mathrm{v})$ was prepared in triplicate for $\mathrm{pH}$ measurement to determine the effect of $\mathrm{pH}$ on solubility of the solid dispersions. The $\mathrm{pH}$ of liquid samples before spray drying was also measured.

\subsubsection{Shape and surface morphology}

The photomicrographs of furosemide, OSA starch, solid dispersion powders and physical mixture were obtained by environmental scanning electron microscope (SEM) with backscattered electron detector (QUANTA ${ }^{\mathrm{TM}}$, FEI, Hillsboro, OR). Powders were mounted on a double faced adhesive carbon tape; then low vacuum (50 and $80 \mathrm{~Pa}$ ) was applied in the chamber, allowing us to observe the powders without further treatment. The voltage was $10 \mathrm{kV}$, working distance was 10 to $8 \mathrm{~mm}$, spot size was 3 and the magnification was from $250 \mathrm{X}$ to $5000 \mathrm{X}$.

\subsubsection{Crystallinity, thermal and spectroscopic properties}

The crystallinity of the API, OSA starch, physical mixture and solid dispersions were assessed by X-ray powder diffraction (XRPD). The experiment was conducted at ambient temperature using monochromatic $\mathrm{Cu} \mathrm{K}$ radiation at $40 \mathrm{~mA}$ and $40 \mathrm{kV}$ in the range of $5^{\circ} \leq 2 \theta \leq 30^{\circ}$ with an angular rate $0.02^{\circ} / \mathrm{s}$. Thermal properties of these samples were obtained by a differential scanning calorimeter with modulated temperature (DSC Q2000, TA Instruments, DE). The samples of around $5 \mathrm{mg}$ were placed in aluminum pans and were tested by a modulated temperature DSC program: equilibrated at $30^{\circ} \mathrm{C}$, scanning from 30 to $250{ }^{\circ} \mathrm{C}$ with a heating rate of $2{ }^{\circ} \mathrm{C} / \mathrm{min}$, a period of $60 \mathrm{~s}$ and amplitude of $\pm 0.32{ }^{\circ} \mathrm{C}$. Fourier transform-infrared spectra were obtained on a FT-IR spectrometer (Perkin Elmer, Waltham, MA). The sample powder was measured directly with no additional preparation.

\subsubsection{Humidity effect on solid dispersion and Dynamic Vapor Sorption (DVS)}

DVS experiment was conducted to characterize if there is humidity induced crystallization and hygroscopicity of samples. The experiments were undertaken using a Dynamic Vapor Sorption apparatus (DVS, Surface Measurement Systems, London, UK). The instrument has a microbalance inside a temperature-controlled cabinet. Temperature was kept at $25^{\circ} \mathrm{C}$ for all experiments with dry nitrogen gas purge at $200 \mathrm{ml} / \mathrm{min}$. The rate and extent of water uptake determined over a range of 0 to 90 to $0 \% \mathrm{RH}$ cycle. The equilibrium was assumed when the weight change was less than $0.02 \% \mathrm{w} / \mathrm{w}$. The time for each sample varied from 5-10 hrs. 
For control I, one more DVS experiment was done to demonstrate humidity induced recrystallization from a amorphous furosemide salt. The sample was treated under dry condition $(0 \% \mathrm{RH})$ for $1 \mathrm{~h}$, and then was treated with constant moisture $(75 \% \mathrm{RH})$ under isothermal $\left(25^{\circ} \mathrm{C}\right)$ environment for $5 \mathrm{hrs}$. Then the sample was collected to run MDSC or DVS with a fixed relative humidity $(75 \% \mathrm{RH}$ for salt control I).

\subsubsection{Physical stability}

Solid dispersion physical stability was performed at $40{ }^{\circ} \mathrm{C}$ for 3 months, at $40{ }^{\circ} \mathrm{C} /$ $75 \% \mathrm{RH}$ for 2.5 months and ambient condition $\left(25 \pm 5^{\circ} \mathrm{C}, 25-55 \% \mathrm{RH}\right)$ for 4 months. XRPD was performed as 2.6 to observe if crystal grew in these samples.

\subsubsection{Dissolution}

Simulated gastric fluid ( $\mathrm{pH}$ 1.2) and phosphate buffer $(\mathrm{pH} 5.8)$ were prepared for dissolution tests. The experiments were conducted at $37^{\circ} \mathrm{C}$ using the USP II method with $100 \mathrm{rpm}$ paddle stirring speed and medium volume of 900 or $600 \mathrm{ml}$. Solid dispersions equivalent to $20 \mathrm{mg}$ furosemide plus other excipients were compressed into tablets using Carver press (Wabash, IN). Tablet weight and formulation ingredients were shown in Table 3-1. Compression force was $1.1 \mathrm{mTon}$; dwelling time was 3 seconds. The punch size was 3/8 inch. The percentage drug release was monitored using an on-line UV-Fiber

optic dissolution system (Dynamic Dissolution Monitor ${ }^{\mathrm{TM}}$ system, Woodbridge Scientific LLC, CT).

Table 3-1. Ingredients of two tablet formulations.

\begin{tabular}{cccc}
\hline Formulation I & \% & Formulation II & \% \\
\hline Solid dispersion powder & 64.5 & Solid dispersion powder & 64.5 \\
Starch 1500 & 32 & MCC & 27 \\
Ai-Di-Sol & 3 & Ai-Di-Sol & 3 \\
MgSt & 0.5 & MgSt & 0.5 \\
& & Colloid silicon dioxide & 5 \\
\hline
\end{tabular}

Tablet weight: $35 \mathrm{mg}$, salt control I, $65 \mathrm{mg}$, corresponding to solid dispersion (50\% drug loading), 105mg, corresponding to solid dispersion (30\% drug loading), 310mg, corresponding to solid dispersion (10\% drug loading). 


\subsection{Results and Discussion}

\subsubsection{Aqueous solubility and $1 \%$ dispersion $\mathrm{pH}$}

Aqueous solubility of all samples was shown in Figures 3-1, 3-2 and 3-3. In each figure, the solubility of API was also shown. The summary of all the solubility and $\mathrm{pH}$ of $1 \%$ dispersion $(\mathrm{w} / \mathrm{v}$ ) was shown in Table 3-2. For salt control samples, four molar ratios between furosemide and ammonia were screened to select a proper ratio that can increase the solubility of API to the highest degree. Ammonia acted as a base toward water to form a mixture of the ammonium and hydroxide ions. Then the oxygen of furosemide will combine ammonium ions to form salt. The reaction can be representing as Figure 3-4.

Theoretically, the reaction of furosemide and ammonia was in 1:1 molar ratio. But practically, the molar ratio 1:1.4 was the minimum amount of ammonia needed to dissolve furosemide. When amount of ammonia increased, the solubility increased, too. However, the solubility did not continually increase when the molar ratio increased from $1: 6$ to $1: 17.9$. The molar ratio that can enhance solubility most should be in the range of $1: 1.4$ to $1: 6.0$. From the present results, the molar ratio $1: 3.6$ was the best one. This phenomenon can be explained as: at a proper ratio, furosemide formed salt with ammonium in solution and after spray drying process, the excess amount of free ammonia will be removed. However, the purpose of the present study was to test the effect of OSA starch on solid dispersion, so the molar ratio 1:1.4 was selected to prepare solid dispersion samples. The $\mathrm{pH}$ of $1 \%$ furosemide dispersion was about 4.8 , the $\mathrm{pH}$ of $1 \%$ dispersion of spray dried salt control samples increased at least one unit and solubility increased about 11 to 48 fold. The salt form contributed to enhanced solubility, and additional discussion of the control samples will be shown later.

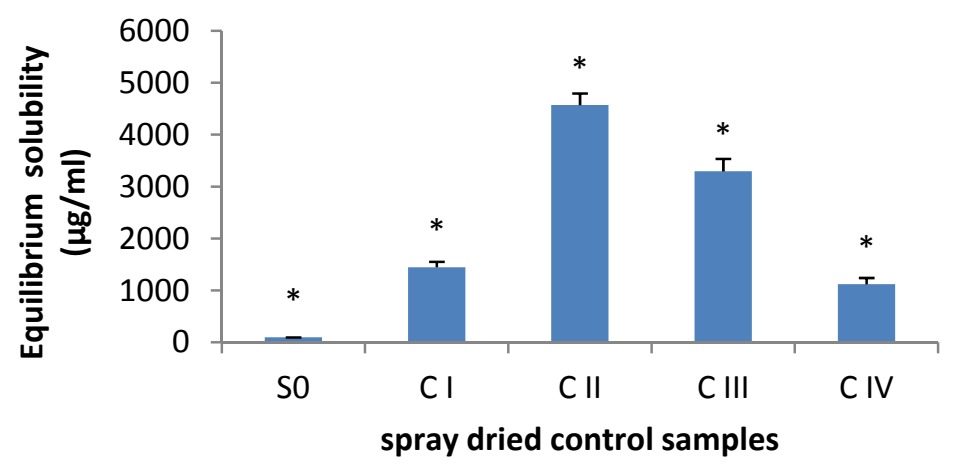

Figure 3-1. Aqueous solubility of controls.

S0: aqueous solubility of furosemide, CI: salt control I, API: ammonia 1:1.4 (mol $/ \mathrm{mol})$; CII: salt control II, API: ammonia 1:3.6 (mol/mol); salt control III, API: ammonia 1:6.0 (mol/mol); salt control IV, API: ammonia 1:6.0 (mol/mol). Each value represents the mean $\pm \mathrm{SD}(\mathrm{n}=3)$. ${ }^{*}$ Each pair, $\mathrm{P}<0.05$, student's t test. 


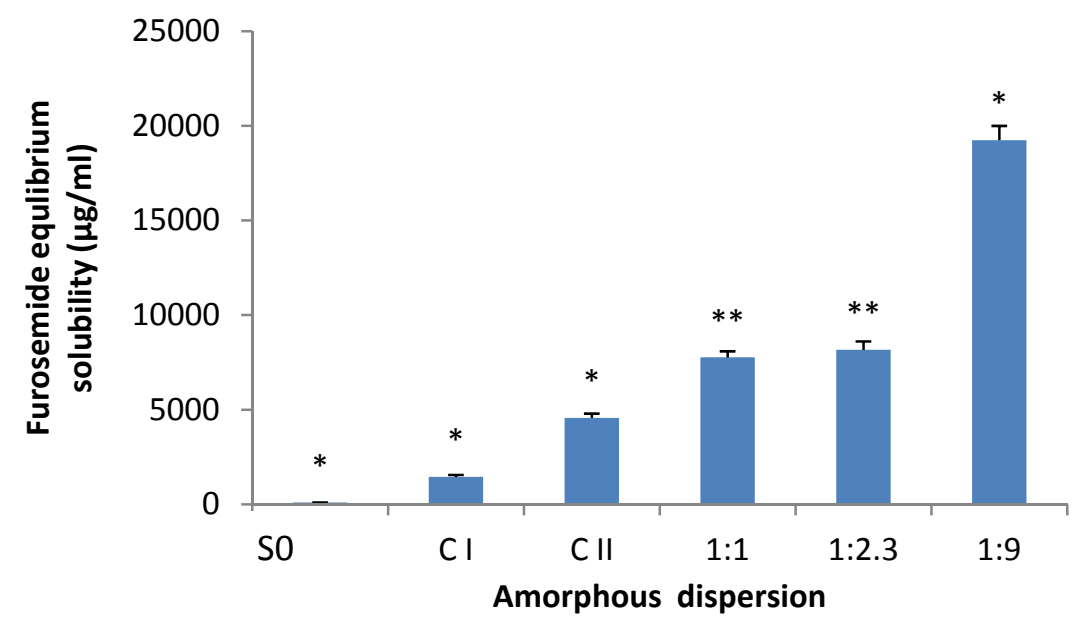

Figure 3-2. Aqueous solubility of amorphous solid dispersions.

S0: aqueous solubility of furosemide; CI: salt control I, API: ammonia 1:1.4 (mol/mol); CII: salt control II, API: ammonia 1:3.6 (mol/mol); 1:1, amorphous solid dispersion API:OSA starch=1:1 (w/w); 1:2.3, amorphous solid dispersion API:OSA starch=1:2.3 $(\mathrm{w} / \mathrm{w}), 1: 9$, amorphous solid dispersion API:OSA starch $=1: 9(\mathrm{w} / \mathrm{w})$. Each value represents the mean $\pm \mathrm{SD}(\mathrm{n}=3)$. * Each pair student's t test, $\mathrm{P}<0.05$ except samples 1:1 and 1:2.3. ${ }^{* *}$ No significant difference between samples: 1:1 and 1:2.3.

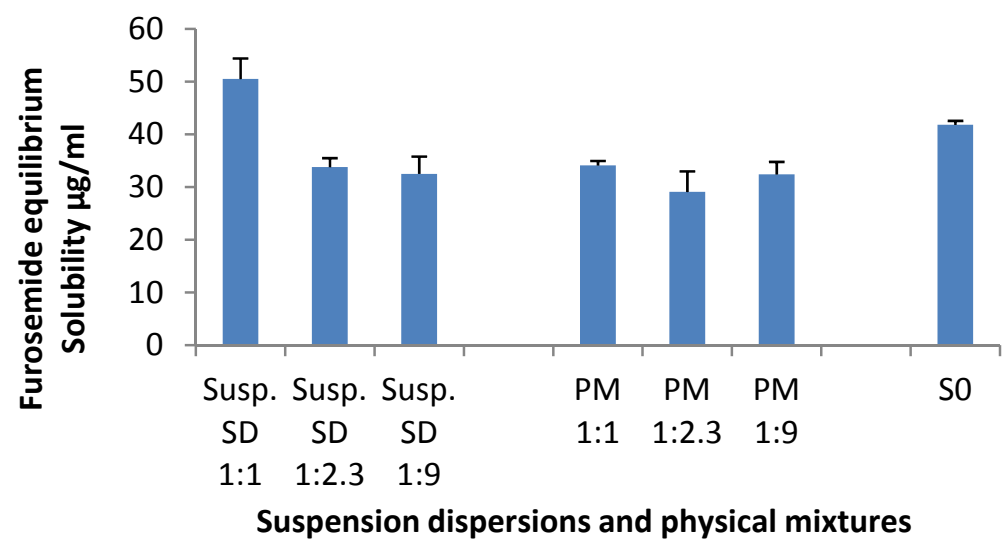

Figure 3-3. Aqueous solubility of suspension solid dispersions and physical mixtures.

S0: aqueous solubility of furosemide; Susp. SD 1:1, spray dried suspension solid dispersion, API: OSA starch=1:1 (w/w); Susp. SD 1:2.3, spray dried suspension solid dispersion, API: OSA starch $=1: 2.3(\mathrm{w} / \mathrm{w})$; Susp. SD 1:9, spray dried suspension solid dispersion, API: OSA starch=1:9 (w/w); PM 1:1, physical mixture, API: OSA starch=1:1 (w/w); PM 1:2.3, physical mixture, API: OSA starch=1:2.3 (w/w); PM 1:9, physical mixture, API: OSA starch=1:9 (w/w). Each value represents the mean \pm SD $(n=3)$. 
Table 3-2. Summary of aqueous solubility and $\mathrm{pH}$ of $1 \%$ dispersion (w/v).

\begin{tabular}{ccc}
\hline Samples & $\mathbf{p H}$ & $\begin{array}{c}\text { Aqueous solubility } \\
(\mathbf{m g} / \mathbf{m l}) \text { mean } \pm \text { SD }\end{array}$ \\
\hline API & 4.84 & $0.096 \pm 0.77$ \\
OSA starch & 4 & NA \\
Control I & 6.3 & 1.4 \\
Control II & 6.82 & 4.6 \\
Control III & 6.6 & 3.2 \\
Control IV & 5.96 & 1.1 \\
Soln. SD 1:1 & 6.24 & 7.8 \\
Soln. SD 1:2.3 & 6.06 & 8.2 \\
Soln. SD 1:9 & 5.95 & 19.2 \\
Susp. SD 1:1 & 4.56 & $0.051 \pm 3.9$ \\
Susp. SD 1:2.3 & 4.34 & $0.034 \pm 1.7$ \\
Susp. SD 1:9 & 4.15 & $0.033 \pm 3.3$ \\
PM 1:1 & 4.42 & $0.034 \pm 0.87$ \\
PM 1:2.3 & 4.32 & $0.029 \pm 3.9$ \\
PM 1:9 & 4.2 & $0.032 \pm 2.4$ \\
\hline
\end{tabular}
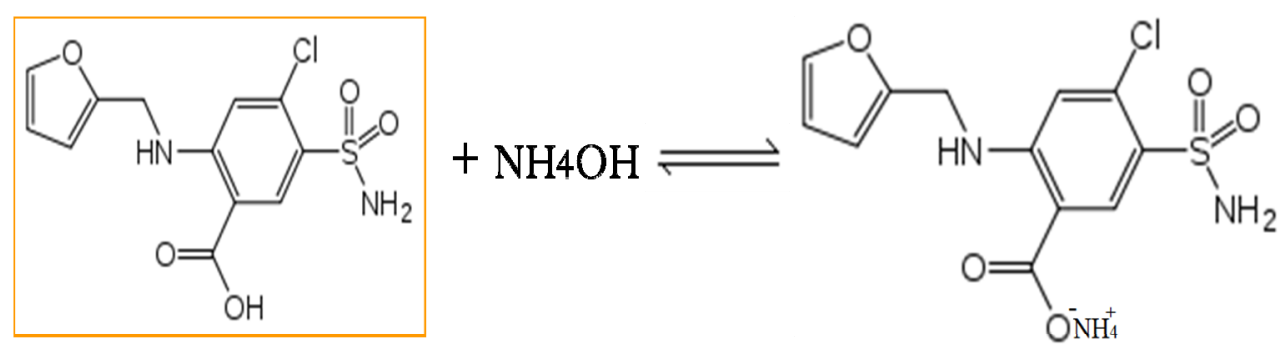

Figure 3-4. Scheme of reaction between furosemide and aqueous ammonia. 
For solution solid dispersion samples, furosemide solubility was increased from $0.096 \mathrm{mg} / \mathrm{ml}, 1.4 \mathrm{mg} / \mathrm{ml}(\mathrm{CI})$ to $19.2 \mathrm{mg} / \mathrm{ml}$ (1:9). Since OSA starch was acidic, the $\mathrm{pHs}$ of solid dispersions were lower than their salt control counterpart. The enhanced solubility did not contribute to $\mathrm{pH}$ increase. Amorphous salt formation should be the major contributor which will be further verified in following discussion.

For suspension solid dispersion and physical mixture, since OSA starch was acidic and furosemide was weak acid, the equilibrium solubility of suspension solid dispersions and physical mixture powders was similar or a little bit lower than that of furosemide. The spray drying process didn't alter the solubility of suspension solid dispersion.

\subsubsection{Shape and surface morphology}

The photomicrographs of furosemide, OSA starch, solid dispersion powders and physical mixture were shown in Figures 3-5 and 3-6. The SEM picture gave the most direct way to show surface morphology and shape of spray dried powders.

The furosemide is a crystallized powder, composed of very small crystals. OSA starch appears as an amorphous spray dried powder.

For the salt control samples: no crystals were found in control I; the two lower ratios (control II and III) powders are composed of small spheres among which we find occasionally a ball that has crystals. For the control IV at low magnification aspect of the powder appears to be identical to the previous (picture didn't illustrate) but with high magnification we see that all the balls are made of crystals.

Solution solid dispersions: For this series, it is hard to differentiate furosemide and starch. Compared to the control, all solid dispersion samples appeared sphere shape and no furosemide crystals were found in those powders.

Suspension solid dispersion: For a ratio of 1:1, a suspension of furosemide in a starch solution allows to obtain an agglomerated powder. Both products are well mixed. The crystals of furosemide are embedded in the starch matrix. When the ratio increase the dispersion of the crystals is not as good as before. Some particles, usually larger, contain a lot of crystals, others consist only of starch.

Physical mixture: The crystals of furosemide and the balls of starch are well separated.

\subsubsection{Crystallinity (XRPD) and spectroscopic properties (FTIR)}

The XRPD spectrum of furosemide, OSA starch, solid dispersion powders and physical mixture were shown in Figure 3-7 and Figure 3-8. 


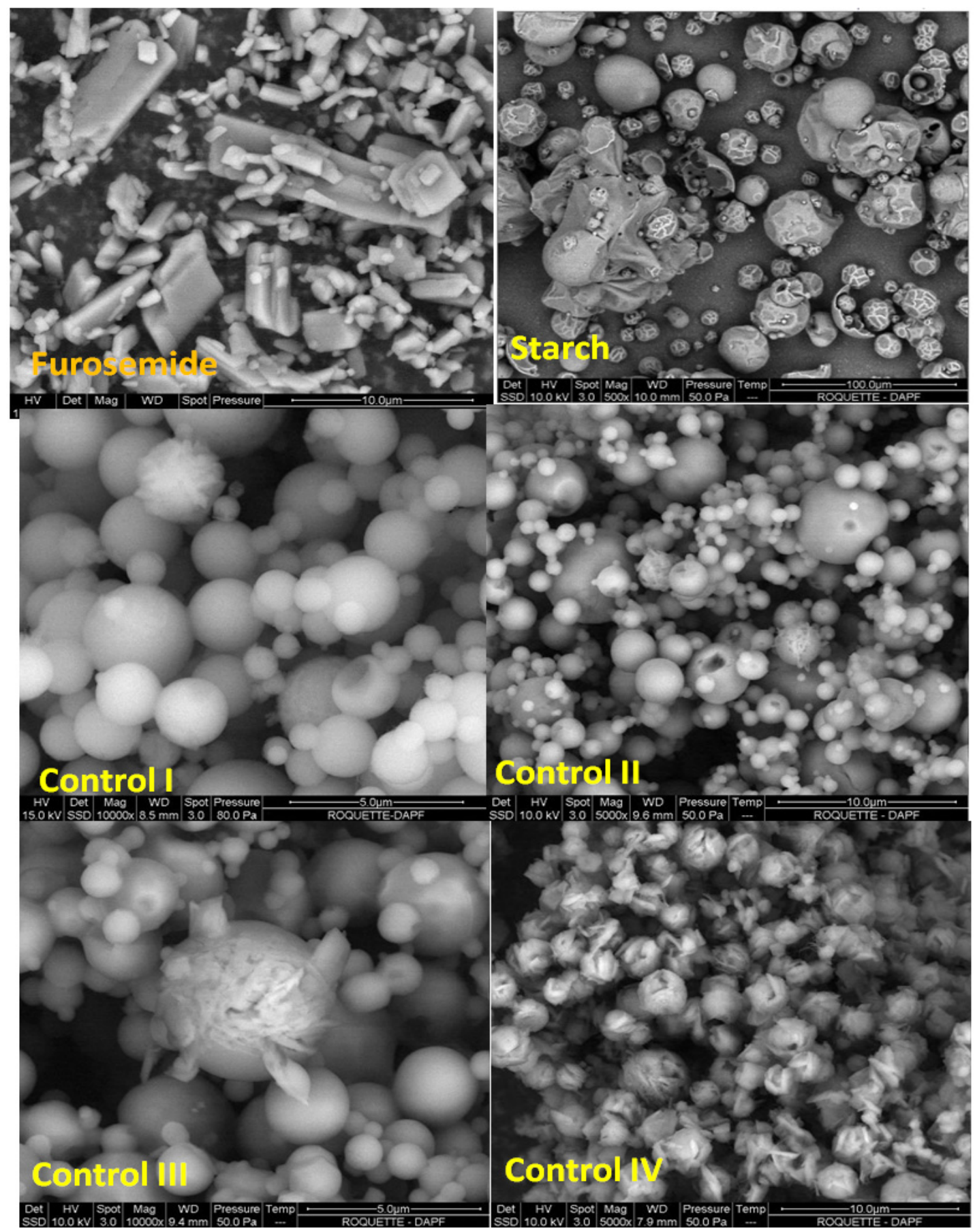

Figure 3-5. SEM pictures (part I) of furosemide powder and OSA starch. Control I: salt control with furosemide to aqueous ammonia in molar ratio 1:1.4 $(10000 \times)$, Control II: salt control with furosemide to aqueous ammonia in molar ratio 1:3.6 (5000×), Control III: salt control with furosemide to aqueous ammonia in molar ratio 1:6.0 (10000×), Control IV: salt control with furosemide to aqueous ammonia in molar ratio 1:17.9 (5000×). All controls were solid dispersion of ammonium salts of furosemide. 
Figure 3-6. SEM pictures (part II) of amorphous solid dispersion with furosemide to OSA starch at different ratio.

$1: 1$, amorphous solid dispersion with furosemide to OSA starch $=1: 1(\mathrm{w} / \mathrm{w})(10000 \times)$;

$1: 2.3$, amorphous solid dispersion with furosemide to OSA starch $=1: 2.3(\mathrm{w} / \mathrm{w})(5000 \times)$;

1:9, amorphous solid dispersion with furosemide to OSA starch $=1: 9(\mathrm{w} / \mathrm{w})(5000 \times)$;

Susp 1:1, crystal dispersion with furosemide to OSA starch $=1: 1(\mathrm{w} / \mathrm{w})(1000 \times)$;

Susp 1:2.3, crystal dispersion with furosemide to OSA starch $=1: 2.3(\mathrm{w} / \mathrm{w})(1000 \times)$;

Susp 1:9, crystal dispersion with furosemide to OSA starch $=1: 9(\mathrm{w} / \mathrm{w})(1000 \times)$;

PM 1:1, physical mixture with furosemide to OSA starch $=1: 1(\mathrm{w} / \mathrm{w})(1000 \times)$. 


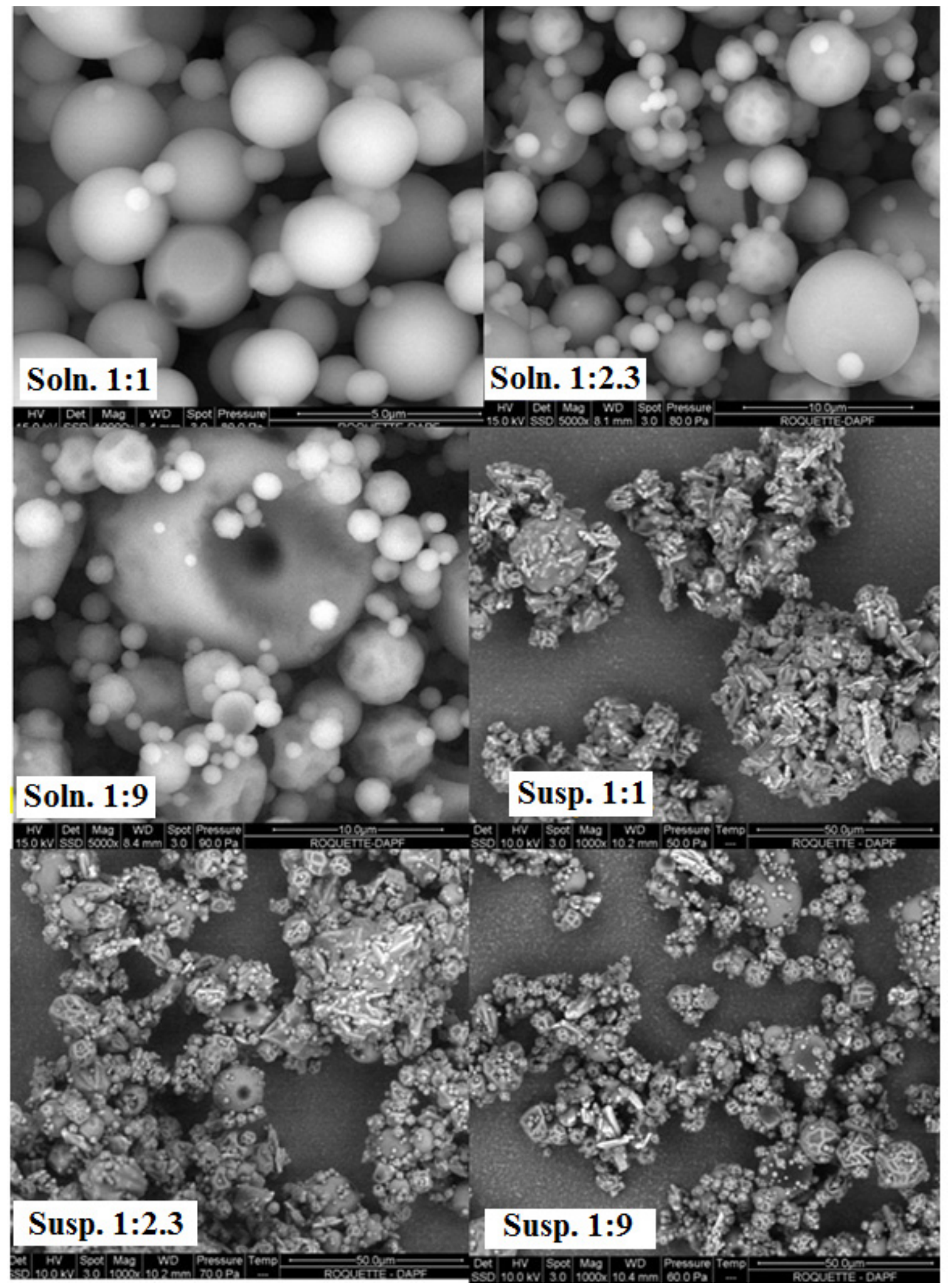




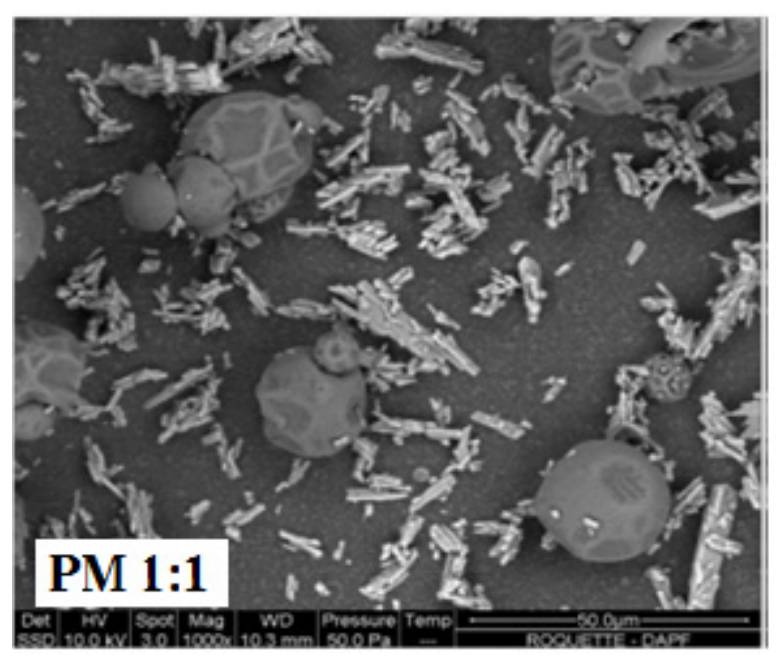

Figure 3-6. (Continued). 

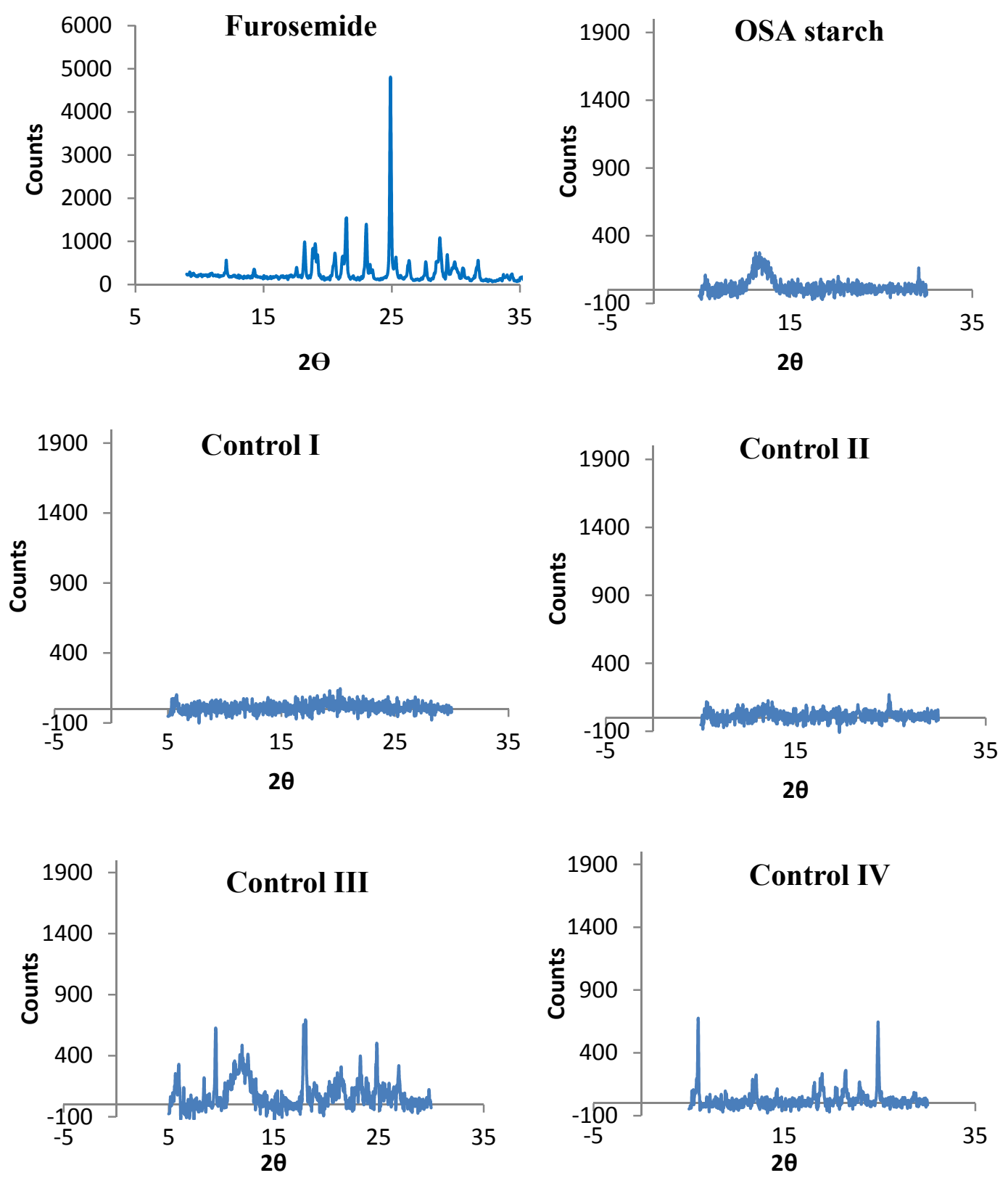

Figure 3-7. XRPD spectrum (part I) of furosemide powder and OSA Starch. Control I: salt control with furosemide to aqueous ammonia in molar ratio 1:1.4, Control II: salt control with furosemide to aqueous ammonia in molar ratio 1:3.6, Control III: salt control with furosemide to aqueous ammonia in molar ratio 1:6.0, Control IV: salt control with furosemide to aqueous ammonia in molar ratio 1:17.9. All controls were solid dispersion of ammonium salts of furosemide. 
Figure 3-8. XRPD spectrum (part II) of amorphous solid dispersion with furosemide to OSA starch at different ratio.

Soln. SD 1:1 amorphous solid dispersion with furosemide to OSA starch $=1: 1(\mathrm{w} / \mathrm{w})$;

Soln. SD 1:2.3 amorphous solid dispersion with furosemide to OSA starch =1:2.3 (w/w);

Soln. SD 1:9 amorphous solid dispersion with furosemide to OSA starch = 1:9 (w/w);

Susp. SD 1:1 crystal dispersion with furosemide to OSA starch $=1: 1(\mathrm{w} / \mathrm{w})$;

Susp. SD 1:2.3 crystal dispersion with furosemide to OSA starch = 1:2.3 (w/w);

Susp. SD 1:9 crystal dispersion with furosemide to OSA starch $=1: 9(\mathrm{w} / \mathrm{w})$;

PM 1:1 physical mixture with furosemide to OSA starch $=1: 1(\mathrm{w} / \mathrm{w})$;

PM 1:2.3 physical mixture with furosemide to OSA starch $=1: 2.3(\mathrm{w} / \mathrm{w})$;

PM 1:9 physical mixture with furosemide to OSA starch $=1: 9(\mathrm{w} / \mathrm{w})$. 

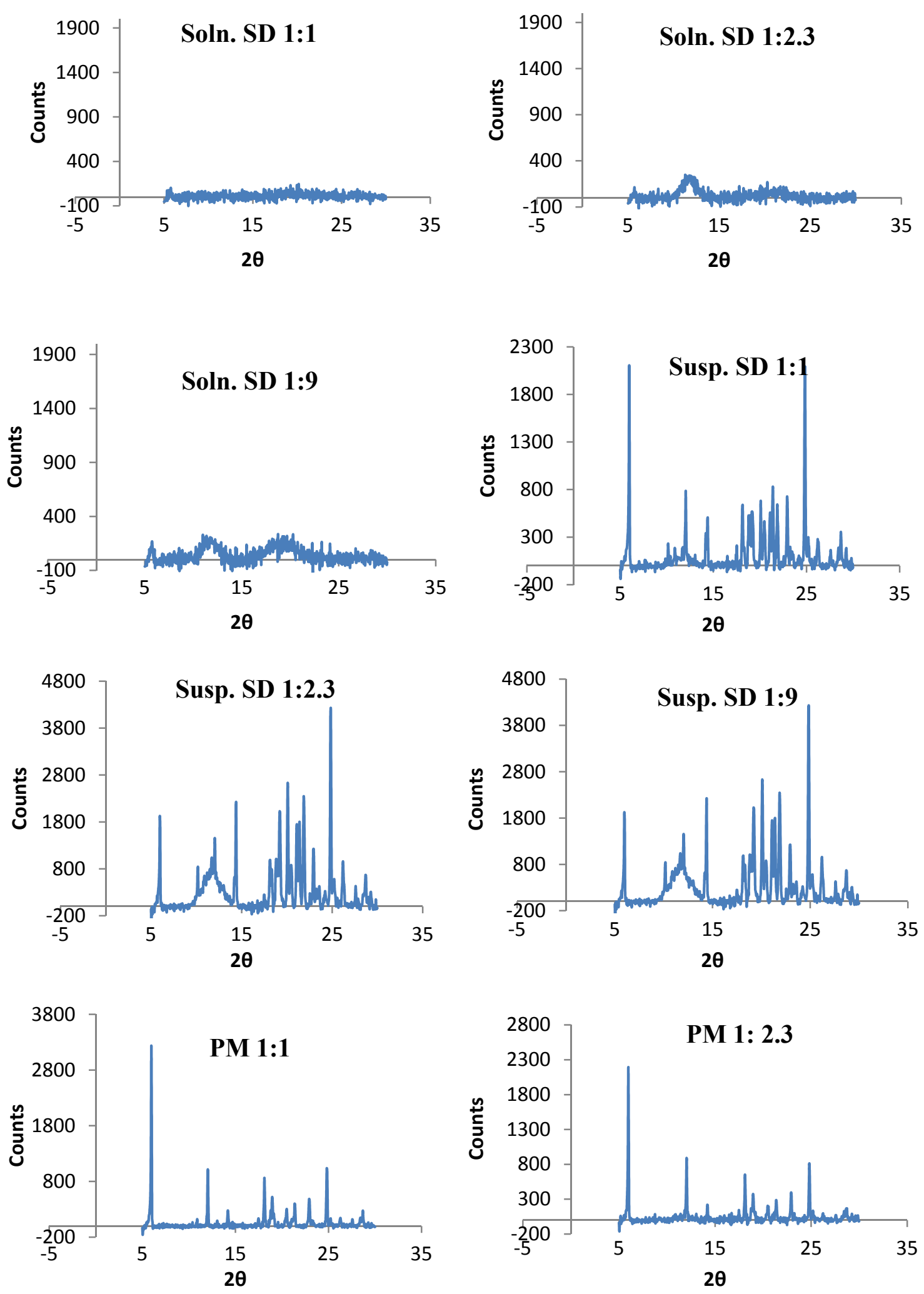


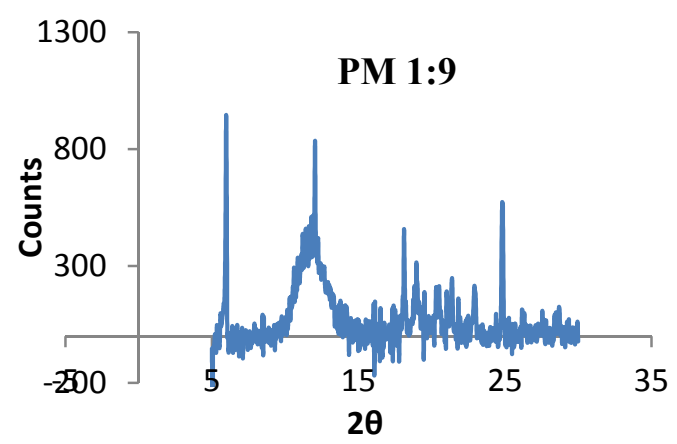

Figure 3-8. (Continued).

Furosemide was crystalline and many sharp peaks are shown in Figure 3-7. The near rectangle shape crystal of furosemide was also shown in the SEM picture. OSA starch basically was an amorphous polymer and no sharp peaks shown in its XRPD spectrum. For the four controls, they are amorphous and crystal salts. Control I and II were amorphous salts, the ratio between aqueous ammonia and furosemide was 1:1.4 $(\mathrm{mol} / \mathrm{mol})$ or 1:3.6 ( $\mathrm{mol} / \mathrm{mol})$. As for control III and IV samples, excess amount of ammonia was added to react with API which was favorable to form pure salt and crystallized in days.

Solution solid dispersions: For this series, all the solid dispersion was amorphous and no sharp peaks shown on their XRPD spectrum (Figure 3-8). The ratio between furosemide and starch was 1:1 (w/w), 1:2.3 (w/w) and 1:9 (w/w). For all the three samples, the ratio between furosemide and aqueous ammonia was 1:1.4 ( $\mathrm{mol} / \mathrm{mol})$.

Suspension solid dispersion: a suspension of furosemide in OSA starch solution allows a liquid dispersion to be obtained. After spray drying the liquid suspension, furosemide didn't change its crystal form. The specific XRPD spectrum of crystalline furosemide is shown in Figure 3-8. Increasing the starch amount didn't affect furosemide changing form from crystal to amorphous.

Physical mixture: furosemide kept its crystalline form in physical mixtures no matter how much OSA starch added into their mixture.

The FTIR spectrums were shown in Figures 3-9 to 3-12. The characteristic absorption bands of furosemide were $\mathrm{C}=\mathrm{O}$ stretching of carboxylic acid group at 1670 $\mathrm{cm}-1 ; \mathrm{N}-\mathrm{H}$ bending at $1591 \mathrm{~cm}-1,1561 \mathrm{~cm}-1 ; \mathrm{S}=\mathrm{O}$ stretching of the sulfonamide group at $1318 \mathrm{~cm}-1$ (asymmetric) and $1140 \mathrm{~cm}-1$ (symmetric); 3350 cm-1 was assigned to the $\mathrm{NH}$ stretching vibration of $\mathrm{Ar}-\mathrm{NHCH}_{2}$ and the $3282 \mathrm{~cm}-1$ band to the stretching vibration of $\mathrm{SO}_{2} \mathrm{NH}_{2}$ [109].

The amorphous information also showed on FTIR spectrum. In the amorphous sample control I and II, even in control III which was less amorphous than that of control 


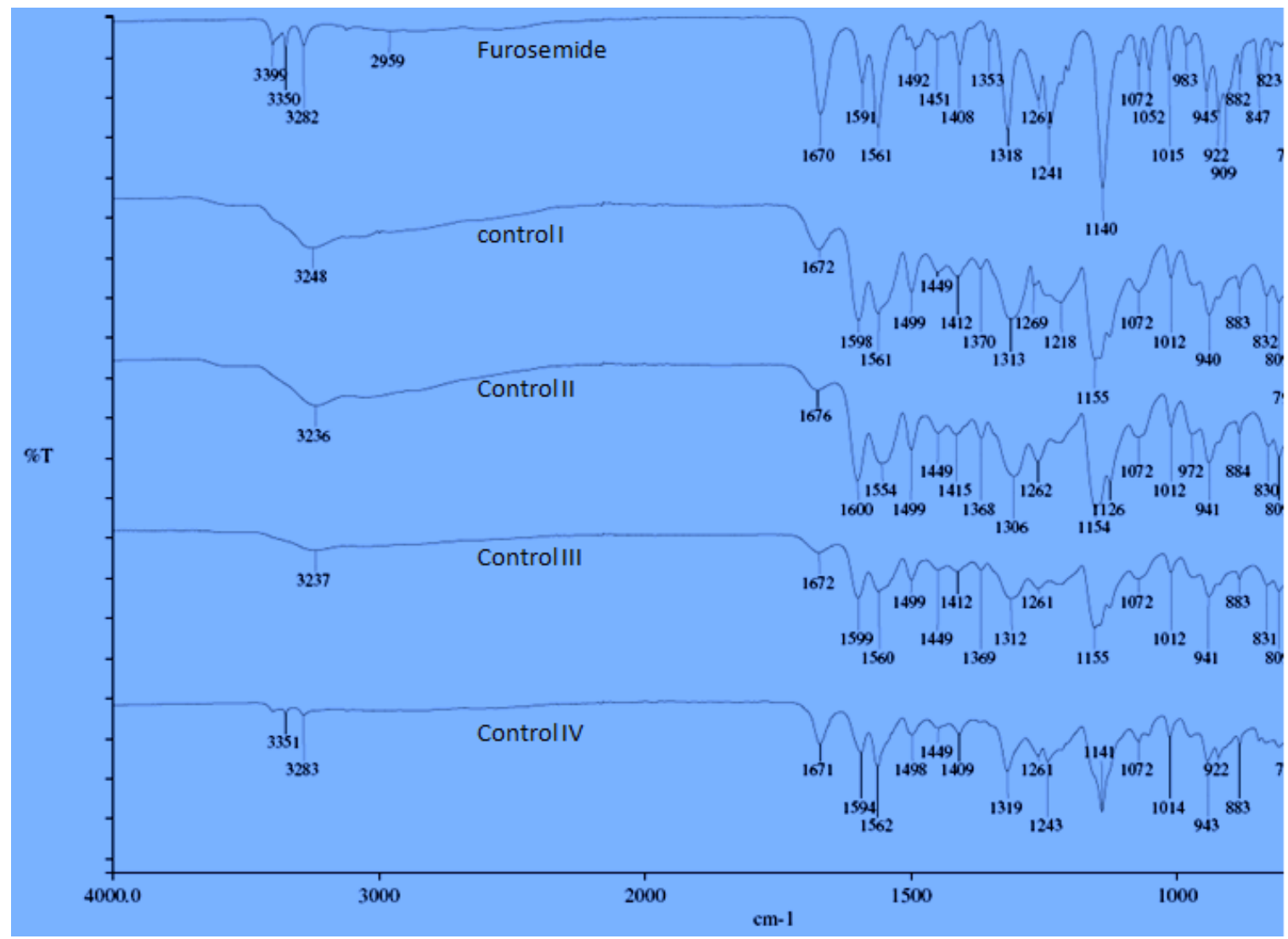

Figure 3-9. FTIR spectrum of furosemide, OSA starch and four controls. 


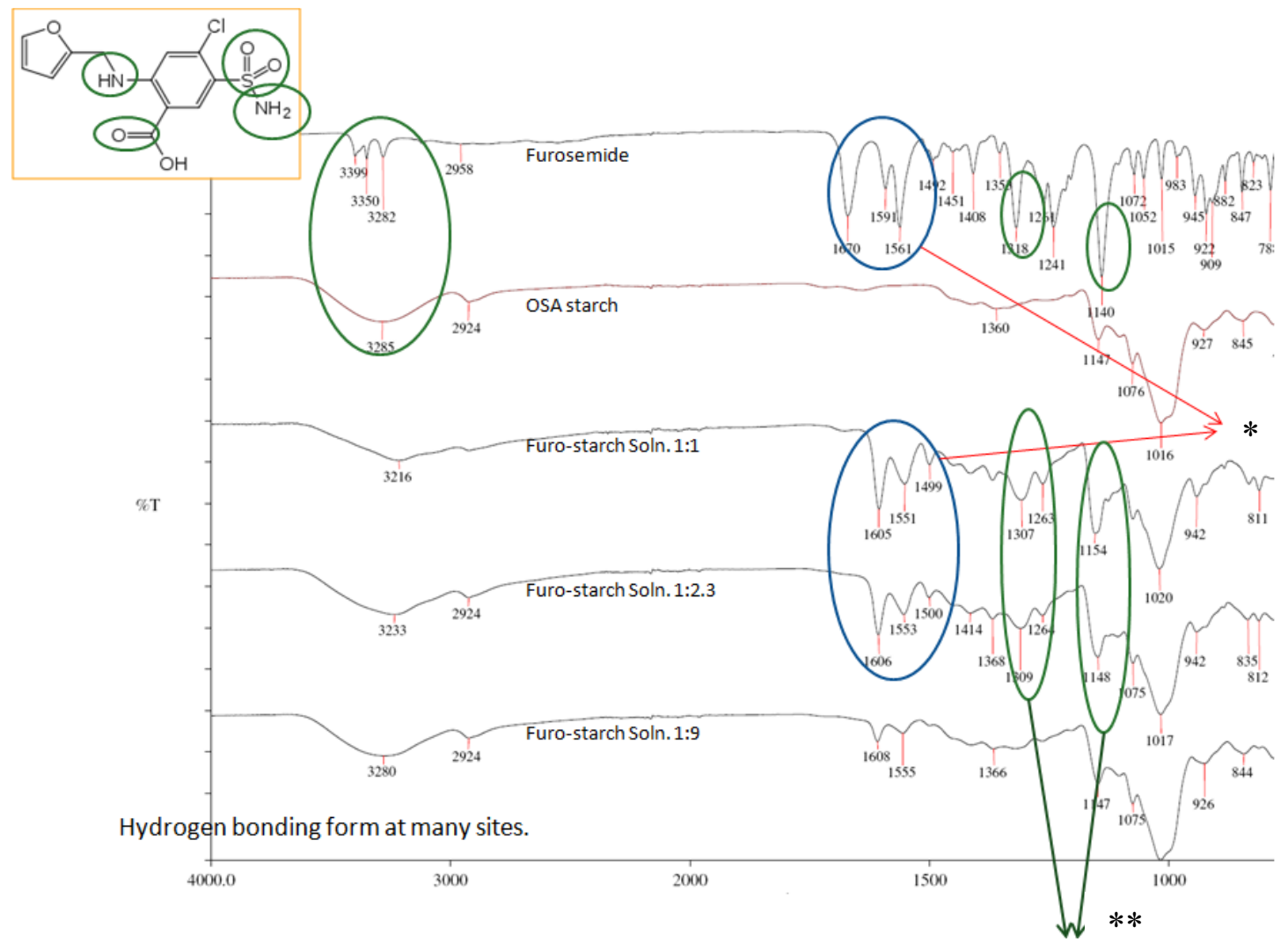

Figure 3-10. FTIR spectrum of furosemide, OSA starch and solution solid dispersions.

*Hydrogen bonding; **Amorphous spectrum. 


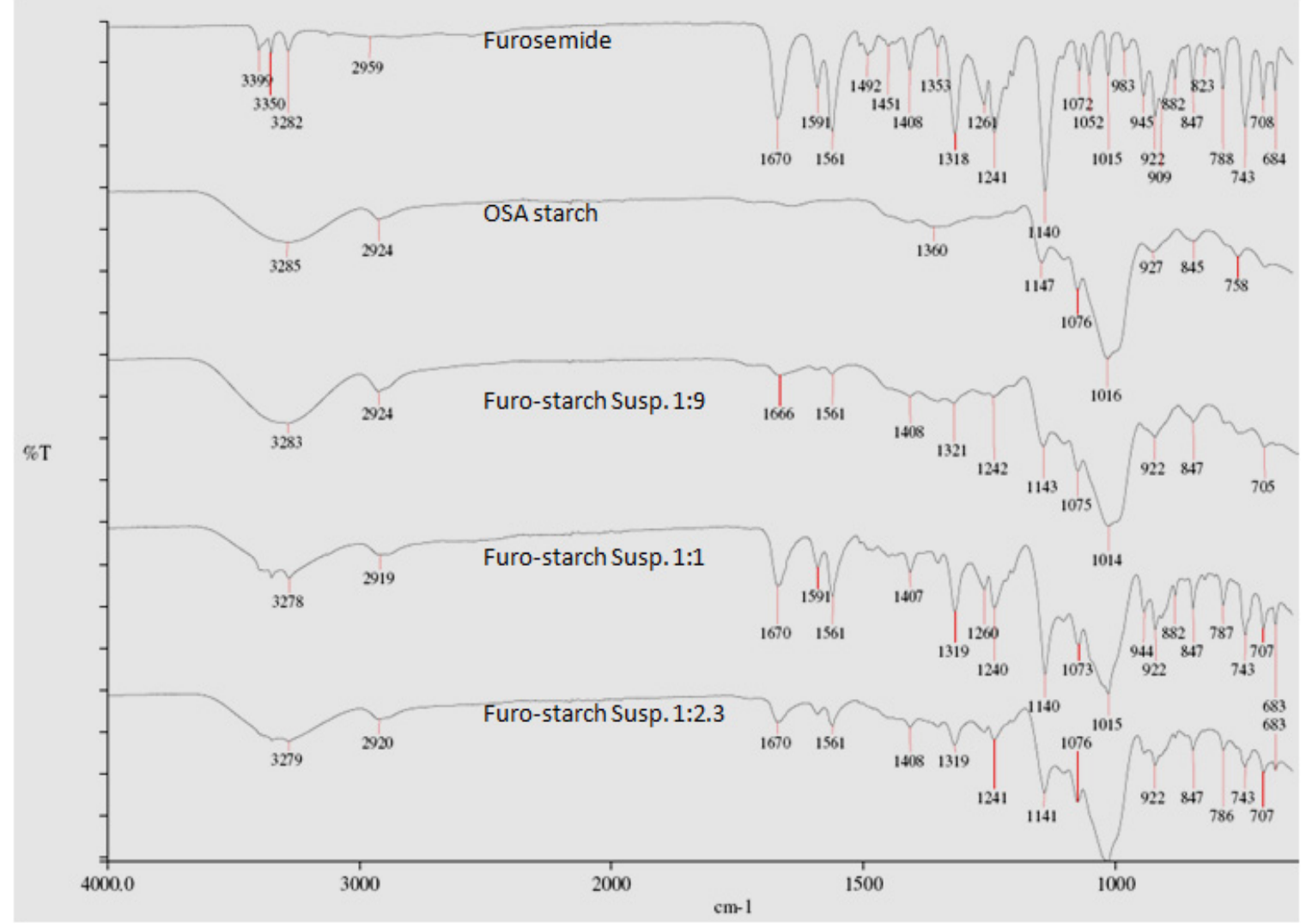

Figure 3-11. FTIR spectrum of furosemide, OSA starch and suspension solid dispersions.

The three solid dispersions were spray dried furosemide-OAS starch suspensions. 


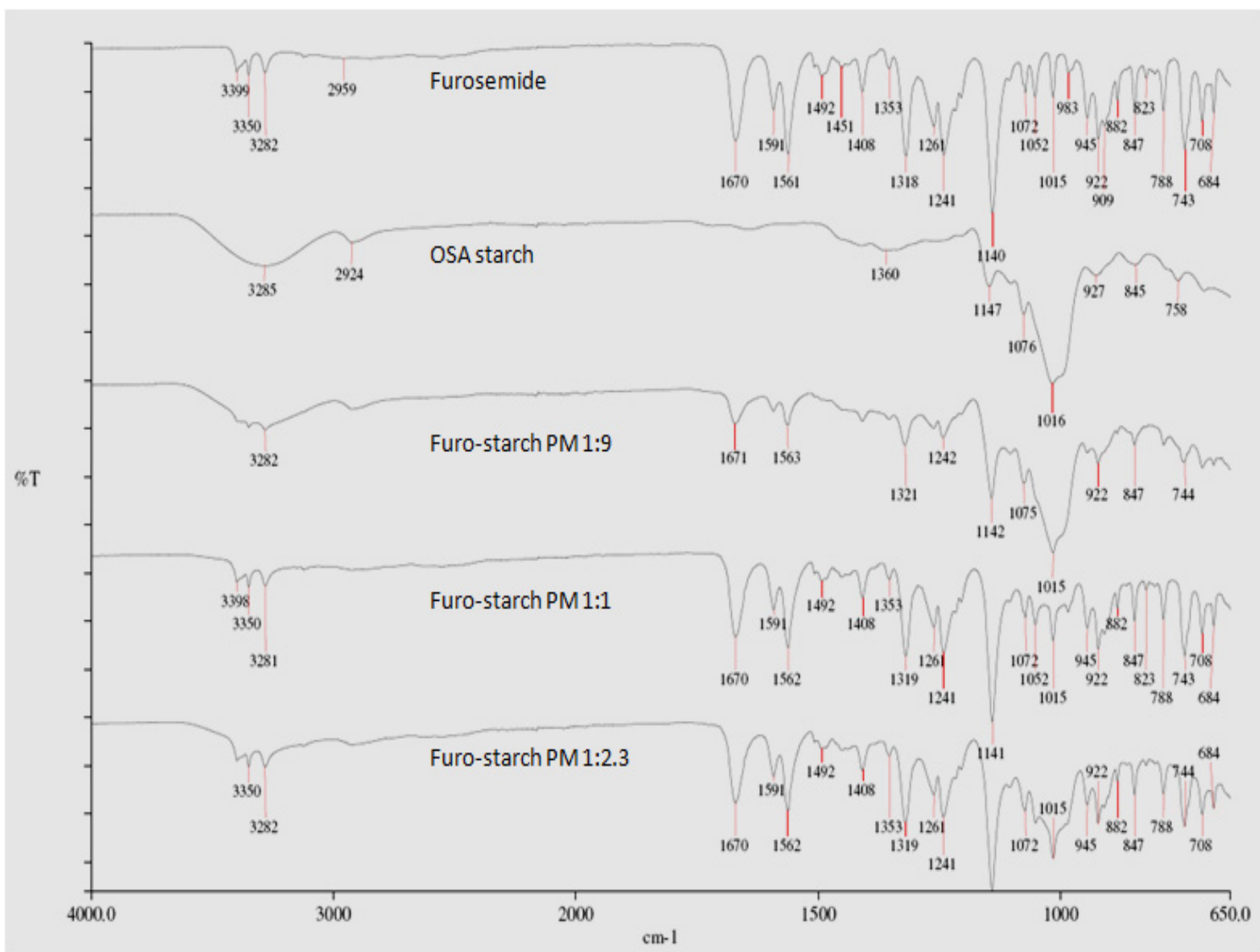

Figure 3-12. FTIR spectrum of furosemide, OSA starch and physical mixtures. 
I and II, the bands at 3399, 3350 and 3282 became a one wide band; at the same time, band at $1140 \mathrm{~cm}-1$ shifted to $1154-1155 \mathrm{~cm}-1$ and become wider; band at $1318 \mathrm{~cm}-1$ shifted to 1306-1312 cm-1 and become wide, while in the crystal control IV, all above mentioned bands do not have shift. One of the reasons for peak shift was that the atoms arrangements in amorphous samples were loose and easily affected by surrounding chemical environment.

Solution solid dispersions: the FTIR spectrum of this series was shown in Figure 3-9. The specific amorphous spectrum was also shown in Furo-starch Soln.1:1; Furostarch Soln. 1:2.3 and Furo-starch Soln. 1:9. Band at $1140 \mathrm{~cm}-1$ shifted to 1147-1154 cmland become wider; band at $1318 \mathrm{~cm}-1$ shifted to $1307-1309 \mathrm{~cm}-1$ and become wider.

The solid dispersion of furosemide-starch yielded band shifted from $1670 \mathrm{~cm}^{-1}$ to 1605 or $1606 \mathrm{~cm}^{-1}$ for $\mathrm{C}=\mathrm{O}$ stretching of carboxylic acid group. It can be interpreted as hydrogen bonding between $\mathrm{C}=\mathrm{O}$ group in furosemide and $-\mathrm{OH}$ group in OSA starch [102].

Suspension solid dispersion: the FTIR spectrum of this series was shown in Figure 3-10. In furosemide-OSA starch 1:1 and 1:2.3 samples, $\mathrm{C}=\mathrm{O}$ stretching of carboxylic acid group at $1670 \mathrm{~cm}-1, \mathrm{~S}=\mathrm{O}$ stretching of the sulfonamide group at 1318 $\mathrm{cm}-1$ (asymmetric) and $1140 \mathrm{~cm}-1$ (symmetric) kept its original position. Furosemide kept its crystalline properties in FTIR spectrum.

Physical mixture: FTIR spectrum of furosemide kept its crystal properties in physical mixtures no matter how much OSA starch added

\subsubsection{Thermal analysis}

DSC technique was widely used in determining melting point of crystal line compounds. When compounds changed form from crystal to amorphous, its melting point will disappear and at the same time, glass transition temperature will display. The temperature modulated DSC (MDSC) can be very efficiently used in the case when the thermodynamically reversible and irreversible processes are to be distinguished. The glass transition was the most frequently studied example, both experimentally and theoretically [110, 111]. The MDSC spectrum of control I was shown in Figure 3-13.

The upper one showed the Tg of amorphous control I sample and the lower one showed the Tg of control I which has recrystallized under high moisture condition (Sample was treated as method 2.7). For the amorphous control I, its $\mathrm{Tg}$ was $92.33^{\circ} \mathrm{C}$ one the reverse heat flow with heat capacity $0.3015 \mathrm{~J} /\left(\mathrm{g}{ }^{\circ} \mathrm{C}\right)$, after $\mathrm{Tg}$, a thermal induced recrystallization peak was shown on the nonreversible heat flow which further verified that high amount amorphous content in control I; for humidity induced recrystallized control I, its $\mathrm{Tg}$ was $103.59^{\circ} \mathrm{C}$ with heat capacity $0.1186 \mathrm{~J} /\left(\mathrm{g}{ }^{\circ} \mathrm{C}\right)$ and no recrystallization peak showed on its non-reversible heat flow. This point also indicated that less amorphous content in humidity treated control I didn't induced a measurable recrystallization peak. Calculated the difference of heat capacity, amorphous content of control I decreased to $38.7 \%$ comparing to that of untreated one (assuming $100 \%$ 

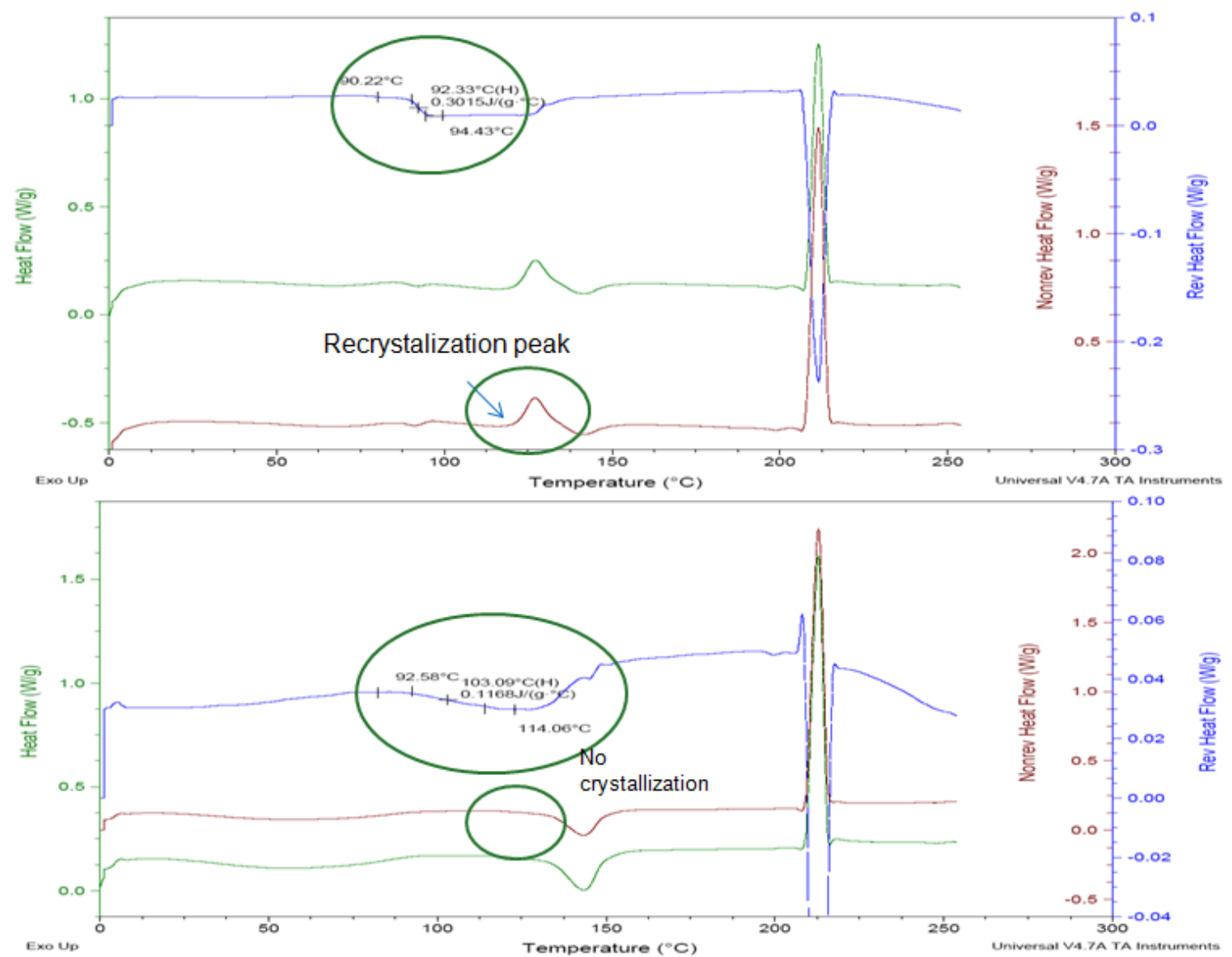

Figure 3-13. MDSC of control I (before and after treated with moisture). 
amorphous content) [112]. Data was shown in Table 3-3. The peak around $216{ }^{\circ} \mathrm{C}$ was furosemide melting combined with decomposition [100].

For the other three control samples, their MDSC were shown in Figure 3-14. On the reverse heat flow spectrum of control II, a clear Tg peak showed and followed this $\mathrm{Tg}$ peak, a clear cold recrystallization peak showed at 114-140 ${ }^{\circ} \mathrm{C}$. Both the symptoms gave strong evidence of an amorphous part in this sample. This result also correlated with those of XRPD and FTIR. As for the control III sample, a very weak Tg peak integrated on the spectrum and no cold crystallization peak can find; similarly, no Tg and crystallization peak can be found in control IV. The MDSC results were in good agreement with those of XRPD. The crystal degree is Control I < Control II $<$ Control III $<$ Control IV. And this point can be showed visually by SEM picture.

For solid dispersions, Tg of each sample was shown in Figure 3-15.Generally, when people make solid dispersion, the high Tg polymer will be considered since it can increase Tg of API. If Tg of solid dispersion is $50{ }^{\circ} \mathrm{C}$ lower than that of API, the amorphous form can be stable theoretically [113]. But sometimes, the pure amorphous form can still crystallized even in very low temperature. So forming solid dispersions with high Tg polymer can stabilize amorphous state and prevent crystallization [44]. For control I, its Tg was $92^{\circ} \mathrm{C}$, while when forming solid dispersion with OSA starch, the Tg was increased to 104,108 and $116^{\circ} \mathrm{C}$ separately for the ratio between furosemide and OSA starch 1:1, 1:2.3 and 1:9 (w/w). Amorphous furosemide should be more stable when it was in solid dispersion, the results of physical stability also prove this point, and this will be further discussed in 3.6.

\subsubsection{Humidity induced crystallization and DVS}

For solid dispersions, one critical issue is to keep drug substance in its amorphous form. But this high energy metastable system is actively prone to rearrange itself to low energy and stable systems: crystal. Moisture and heat are two major induce factors to be considered in this transition. So, in our project, we selected thermal analysis and dynamic vapor sorption analysis to test those amorphous systems. Thermal analysis has been discussed in 3.4, we will focus on effect of humidity on solid dispersion.

OSA starch is hydrophilic material, and at high $\mathrm{RH} \%$, the material absorbed more water vapor. From 0- $50 \mathrm{RH} \%$, some water were entrapped in the starch; from 50 to 90 $\mathrm{RH} \%$, much more water absorbed. By the end of the experiment, 7.6\% bulk water can't be removed and remained in the sample. For furosemide, which is very hydrophobic material, little water was absorbed, even at $\mathrm{RH} \% 90 \%$, the mass increased only $0.14 \%$. The isothermal DVS plot was shown in Figure 3-16.

In this DVS experiment, we treat the amorphous sample with relative humidity range 0 to $90 \%$. A sharp peak in $60 \%$ to $80 \% \mathrm{RH}$ range showed in Figure 3-17. Upon $60 \% \mathrm{RH}$, the amorphous part absorb moisture significantly, the mass gain from $60 \% \mathrm{RH}$ to $70 \% \mathrm{RH}$ reached $3.1 \%$ and at the same time the sample started to crystallize, then water 
Table 3-3. Tg and heat capacity (Cp) of control I (before and after treated with moisture).

\begin{tabular}{cccccc}
\hline Control I & Tg onset & Tg & Tg end & Cp & Amorphous content \\
\hline Before DVS & 90.22 & 92.33 & 94.43 & 0.3015 & $100 \%$ (theoretical) \\
After DVS & 92.58 & 103.59 & 114.06 & 0.1186 & $\sim 38.7 \%$ \\
\hline
\end{tabular}

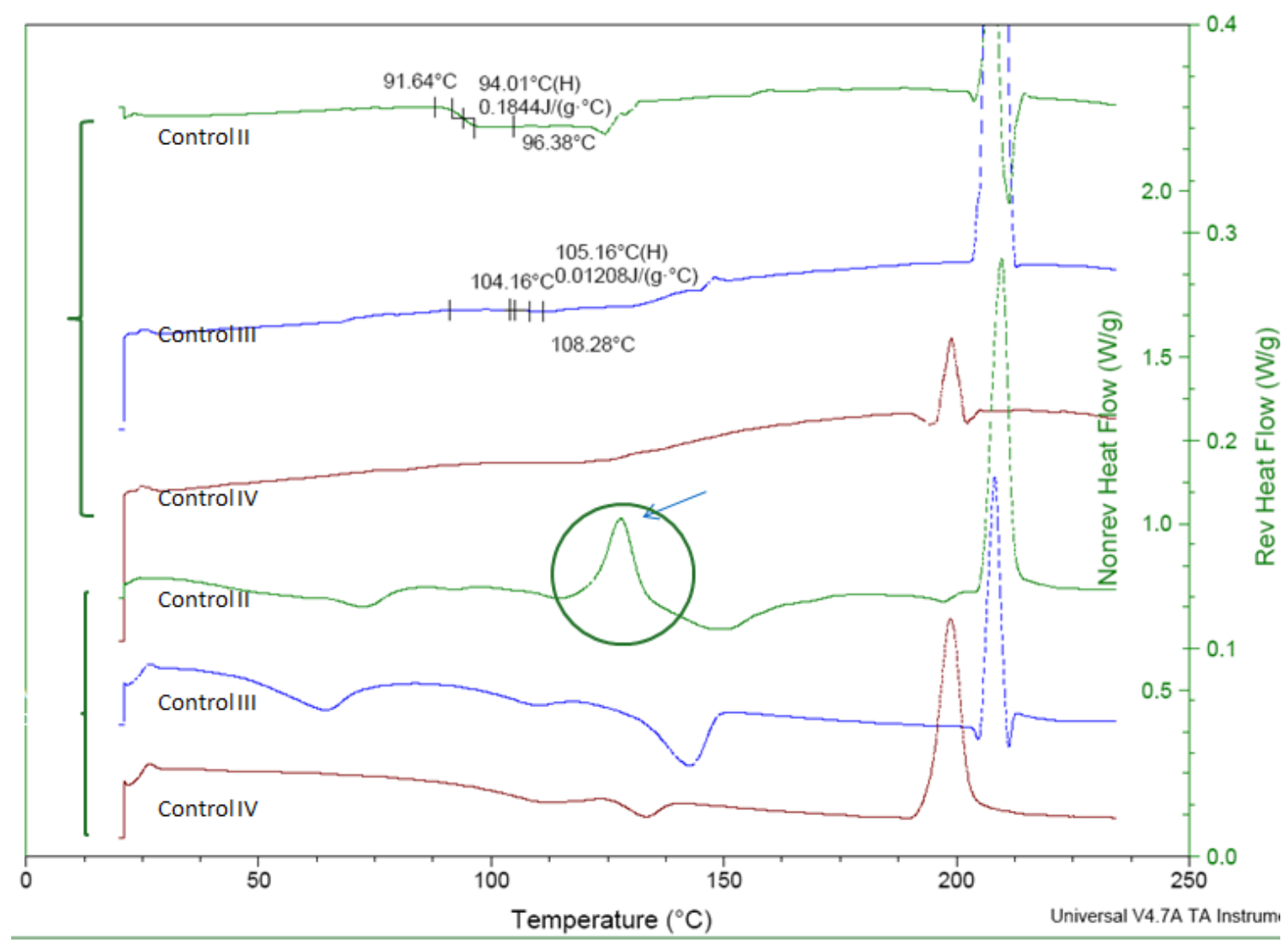

Figure 3-14. MDSC spectrum of control II, III and IV. 


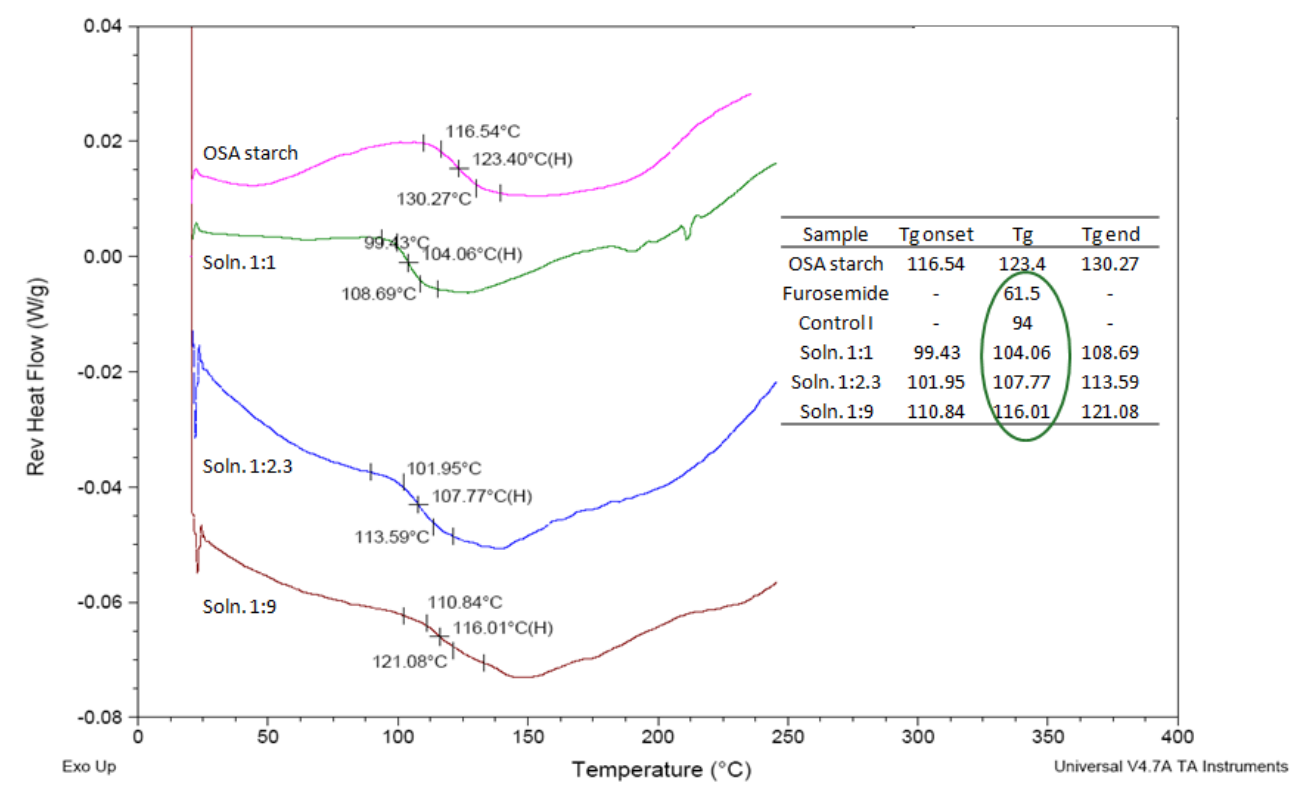

Figure 3-15. Tg of OSA starch, amorphous solid dispersion 1:1, 1:2.3 and 1:9.
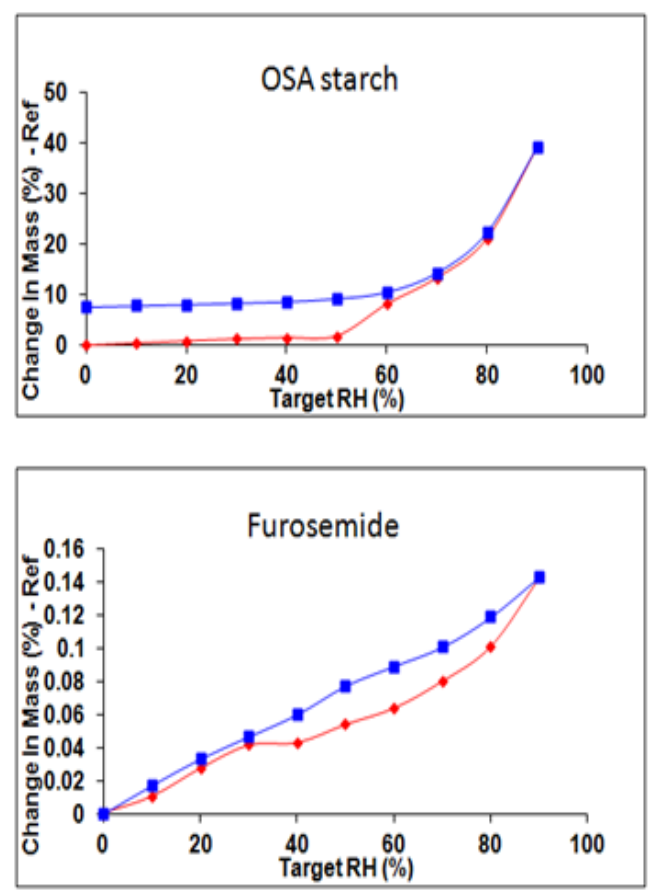

Figure 3-16. DVS plot of OSA starch and furosemide. 

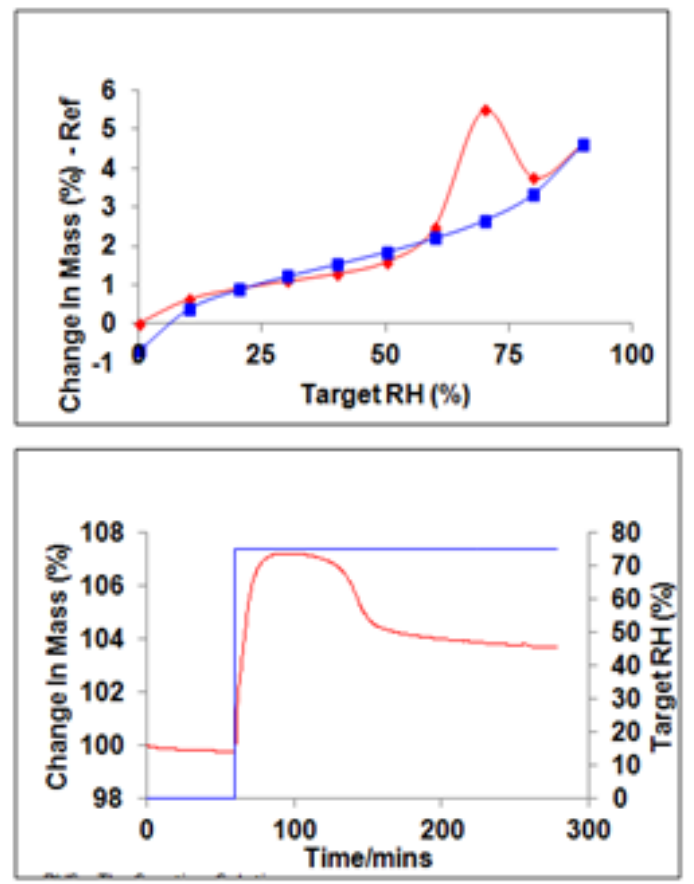

Figure 3-17. Water vapor sorption and desorption graph of control I. 
was eliminated by the crystal as it formed a tight lattice, and the mass decreased sharply. Then the material absorbs moisture gradually until mass reached equilibrium at $90 \% \mathrm{RH}$. In desorption cycle, sample was dried little by little, then reached to $-0.7 \%$ which can be interpreted as water in original sample was removed. Since the $\mathrm{pH}$ of control I didn't change (Table 3-4), this weight loss should not be the ammonia.

When treated amorphous control I under $0 \% \mathrm{RH}$ for 1 hour, then under $75 \% \mathrm{RH}$ for 4 hours, the mass change instantly upon moisture reached to $75 \% \mathrm{RH}$, the mass increased around 90mins, then it become flat. In about one and half hour the moisture induced crystallization finished. Then the sample was collected to run MDSC, results has been discussed in 3.3.4.

The effect of moisture on solution solid dispersions was shown in Figure 3-18. The hygroscopicity of these samples was similar in the relative humidity range of $0-40 \%$. The Low $\% \mathrm{RH}$ values resulted in low vapor sorption and about $2.8 \%$ weight gain to these samples. In the relative humidity range of 40 to $90 \%$, solid dispersion started to absorb water into bulk, weight gain reached to $23,30,40 \%$ for $1: 1,1: 2.3$ and $1: 9$ samples separately. In desorption cycle, the absorbed water can't be removed completely and residual water remained in each sample. This order was highly in accordance to the starch amount in the samples: the higher starch content, the higher water uptake. The water uptake was higher than that of amorphous salt (control I) indicated that furosemide-OSA starch solid dispersion was more hydrophilic and should have higher aqueous solubility than that of amorphous salt. This point was already demonstrated by aqueous solubility results. (Detailed solubility results in 3.1).

\subsubsection{Physical stability test (XRPD)}

Solid dispersion physical stability (XRPD spectrum) at ambient condition (i.e. $25 \pm 5{ }^{\circ} \mathrm{C}, 25-55 \% \mathrm{RH}$ ) for 4 months and $40^{\circ} \mathrm{C}$ for 3 months was shown in Figure 3-19. Physical stability (XRPD spectrum) at $40^{\circ} \mathrm{C} / 75 \% \mathrm{RH}$ for 2.5 months was shown in Figure 3-20.

Solid dispersion kept its amorphous form when sample was treated under ambient condition or $40^{\circ} \mathrm{C}$. During 3 to 4 months storage time, no crystalline was detected by XRPD technique. But when the samples were treated under $40^{\circ} \mathrm{C} / 75 \% \mathrm{RH}$ for 2.5 months, crystalline was detected in all three solid dispersions (XRPD spectrum was shown in Figure 3-20). These phenomena indicated that furosemide-OSA starch solid dispersions were stable to high temperature, but they are fragile to humidity. Amorphous furosemide in solid dispersions was easily recrystallized under high moisture condition. For better application of OSA starch in solid dispersion, it was critical to prevent high moisture environment; specific packaging may used for this purpose 
Table 3-4. pH measurements of control I before and after water vapor sorpdesorp treatment.

\begin{tabular}{cc}
\hline Control I & Value \\
\hline Weight loss $\%$ in desorption & $0.70 \%$ \\
$\mathrm{pH}$ before DVS & $5.63 \pm 0.1$ \\
$\mathrm{pH}$ after DVS & $5.91 \pm 0.19$ \\
\hline
\end{tabular}

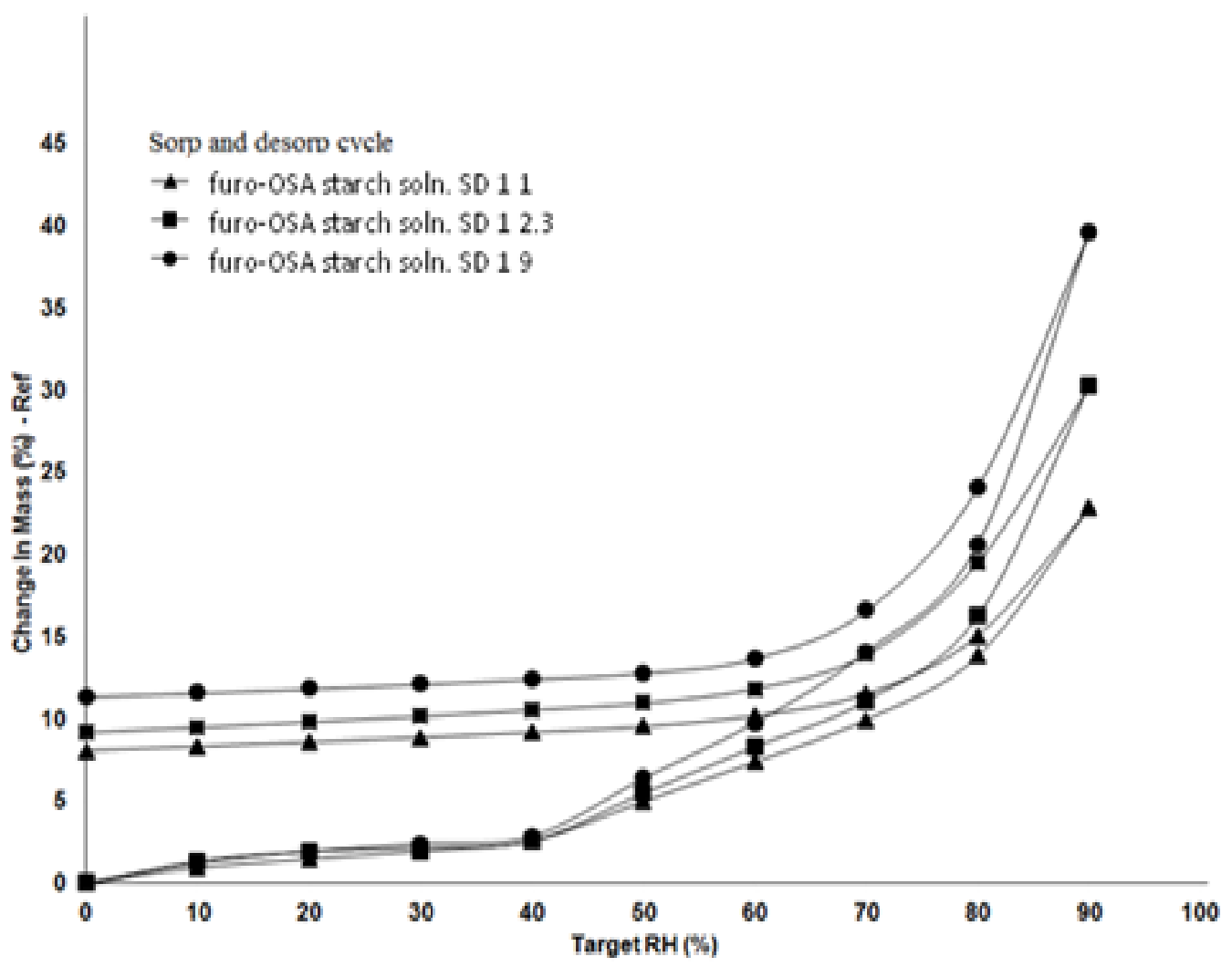

Figure 3-18. DVS graph of solution solid dispersions. 

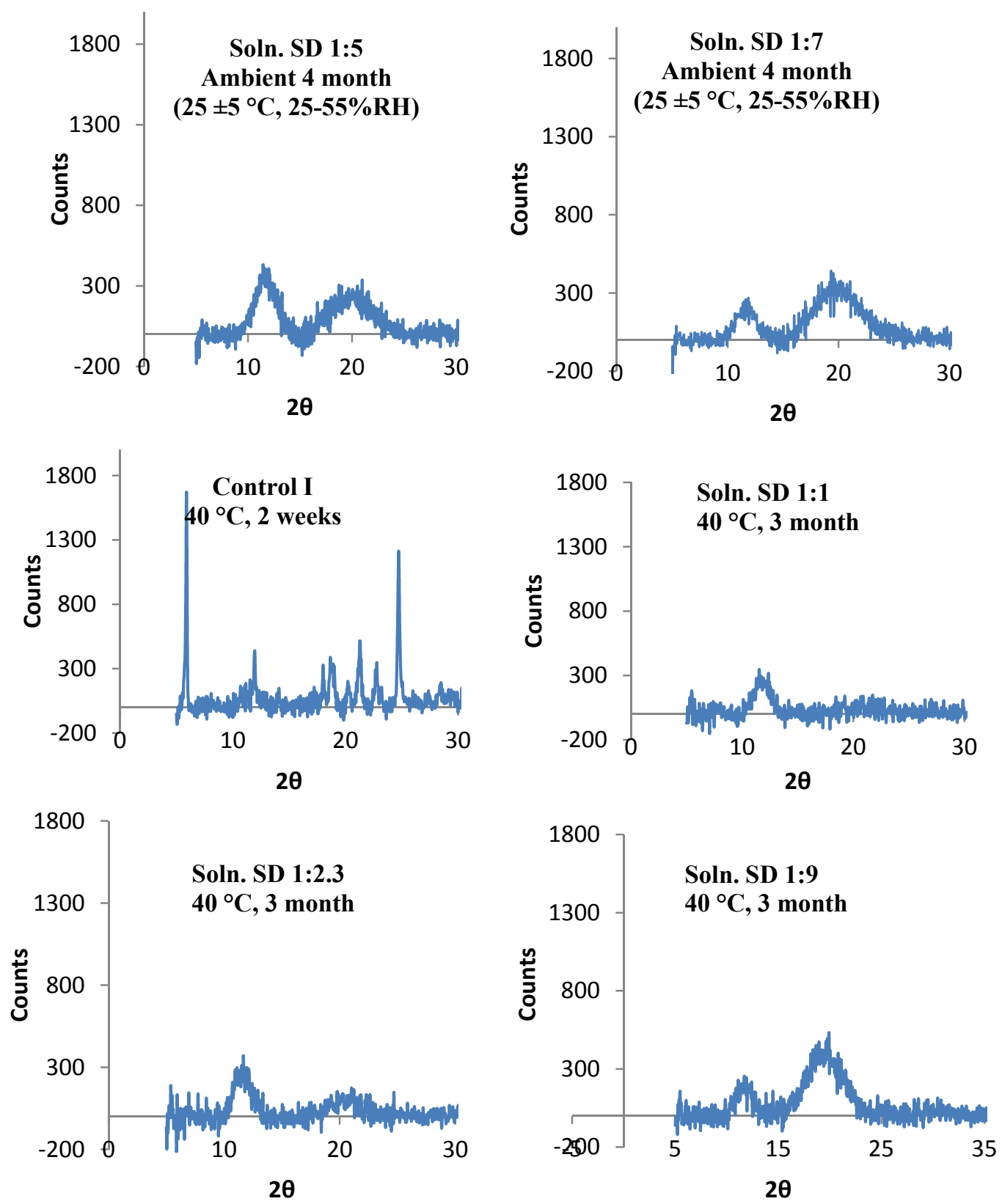

Figure 3-19. Physical stability of solid dispersion at ambient condition and $40{ }^{\circ} \mathrm{C}$. 

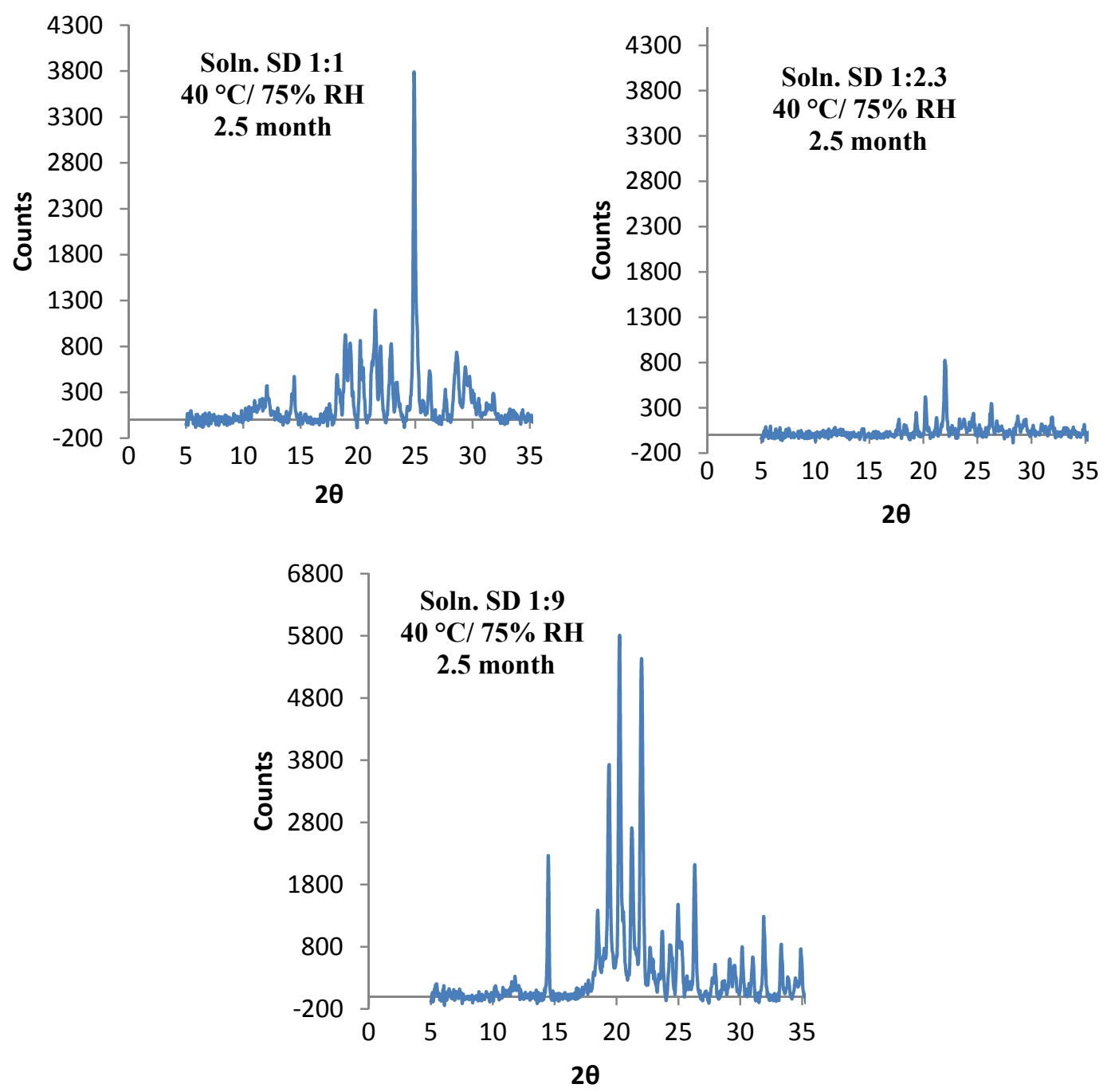

Figure 3-20. Physical stability (XRPD spectrum) of solution solid dispersion under $40{ }^{\circ} \mathrm{C} / 75 \%$ RH for 2.5 months. 


\subsubsection{Dissolution}

For formulation I, drug released completely in 20 min for all test samples in phosphate buffer ( $\mathrm{pH}$ 5.8), but when drug released in gastric acid medium, the drug dissolution slowed down. Around $80 \%$ furosemide released from amorphous solid dispersion 1:9 at $60 \mathrm{~min}$; while for the $2^{\text {nd }}$ one, Lasix $20 \mathrm{mg}$, it released $43 \%$ drug at 60 min point. Drug dissolution in acidic environment was in the order of solid dispersion 1:9 $>$ Lasix $20 \mathrm{mg}>$ Control I > solid dispersion 1:2.3 > solid dispersion 1:1. (Dissolution profile was shown in Figure 3-21). Because furosemide is an acidic compound: it remained essentially unionized when it was in acidic environment which made it less soluble. In this case, salt form didn't help. High amount of OSA starch can keep a supersaturation state of drug in water which increased drug dissolution. However amorphous solid dispersion 1:2.3 and 1:1didn't show a benefit for drug release which may be for two reasons. One was the non-disintegrating property of the tablet which was caused by the stickiness of starch 1500, the other was that the starch amount wasn't enough to maintain supersaturation effectively. Based on the analysis, MCC was selected to replace starch 1500 as filler to prepare formulation II to test if drug can release fast from amorphous solid dispersion: soln. 1:1 and soln. 1:2.3.

For formulation II, drug released completely in 25 min for all test samples in phosphate buffer ( $\mathrm{pH} 5.8$ ), which was similar to that of formulation I. While in SGF, furosemide release was faster from soln. 1:1 and 1:2.3 after using MCC as filler than those using starch1500 as filler. Data is shown in Table 3-5. The results proved the above premise: the less stickiness of MCC helped the solid dispersion tablet to disintegrate and then allowed faster drug release. Dissolution profile of formulation II was shown in Figure 3-22. Faster disintegration can assist drug release but it was less effective in maintaining supersaturation of the drug.

\subsection{Conclusion}

The natural based OSA starch can be used as solid dispersion carrier to prevent drug crystallization, thus maintain an improved solubility and dissolution profile. Humidity proof package material should be considered in OSA starch based solid dispersion dosage form storage to maintain the drug in an amorphous state. Sample preparation methods, drug loading and tablet formulations have significant effect on solubility and dissolution profile of solid dispersions; various techniques including XRPD, SEM, FTIR, MDSC, DVS were applied to elucidate the mechanism of solid dispersion. 

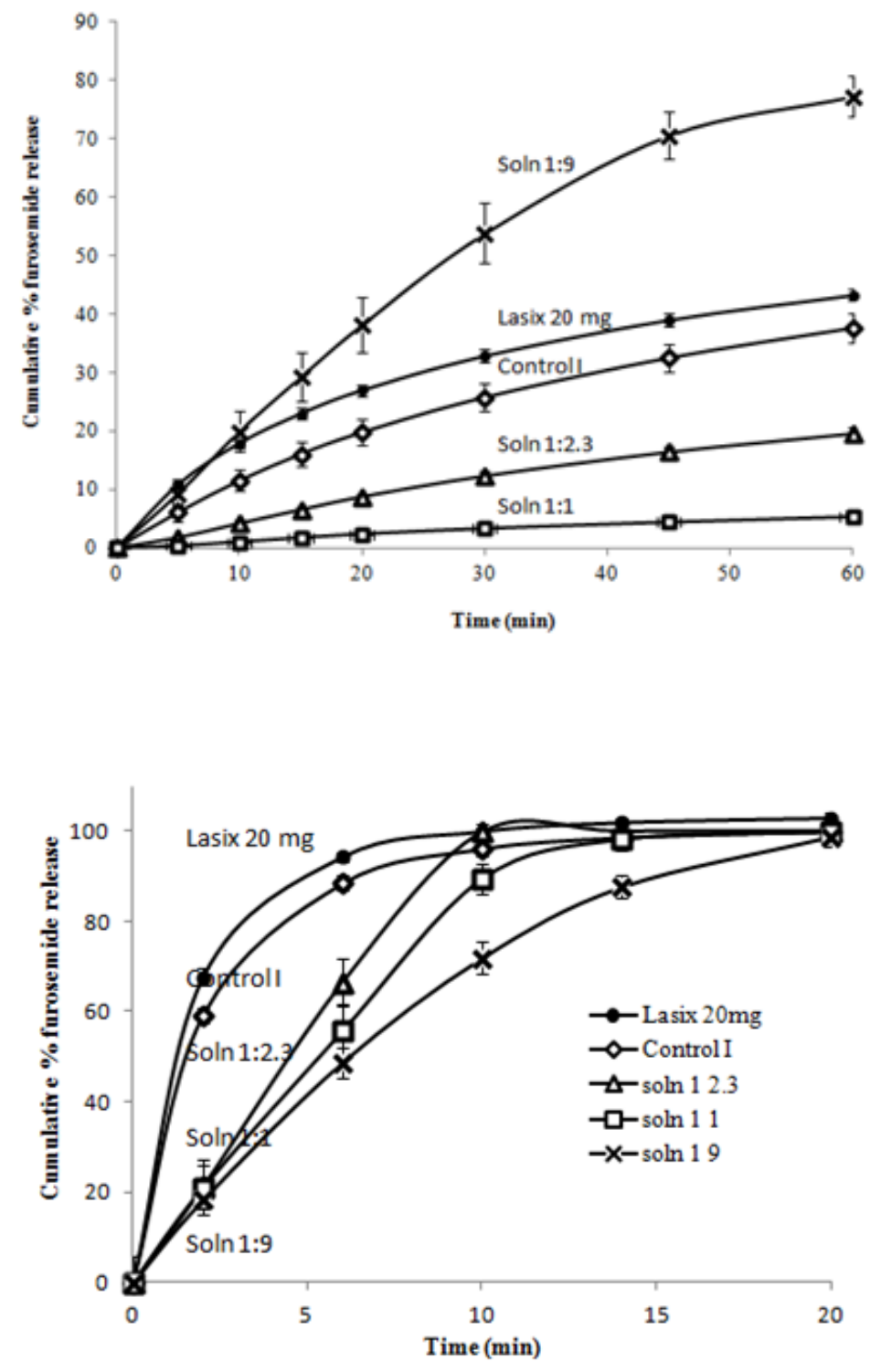

Figure 3-21. Dissolution profile of formulation I in SGF (pH 1.2) and phosphate buffer (pH 5.8).

Soln. SD 1:1 amorphous solid dispersion with furosemide to OSA $\operatorname{starch}=1: 1(\mathrm{w} / \mathrm{w})$; Soln. SD 1:2.3 amorphous solid dispersion with furosemide to OSA starch $=1: 2.3(\mathrm{w} / \mathrm{w})$; Soln. SD 1:9 amorphous solid dispersion with furosemide to OSA starch = 1:9 (w/w); Control I was amorphous furosemide ammonia salt; Lasix $20 \mathrm{mg}$ was commercial furosemide tablet, the strength was $20 \mathrm{mg} /$ tablet. 
Table 3-5. Comparison of \%drug release in two formulations at $60 \mathrm{~min}$ point in SGF (pH 1.2).

\begin{tabular}{cccccc}
\hline Item & Soln. 1:9 & Soln. 1:2.3 & Soln. 1:1 & Control I & Lasix 20 mg \\
\hline Formulation I & 80 & 19.6 & 5.4 & 37 & 43 \\
Formulation II & 80 & 57 & 13 & 2 & 43 \\
\hline
\end{tabular}



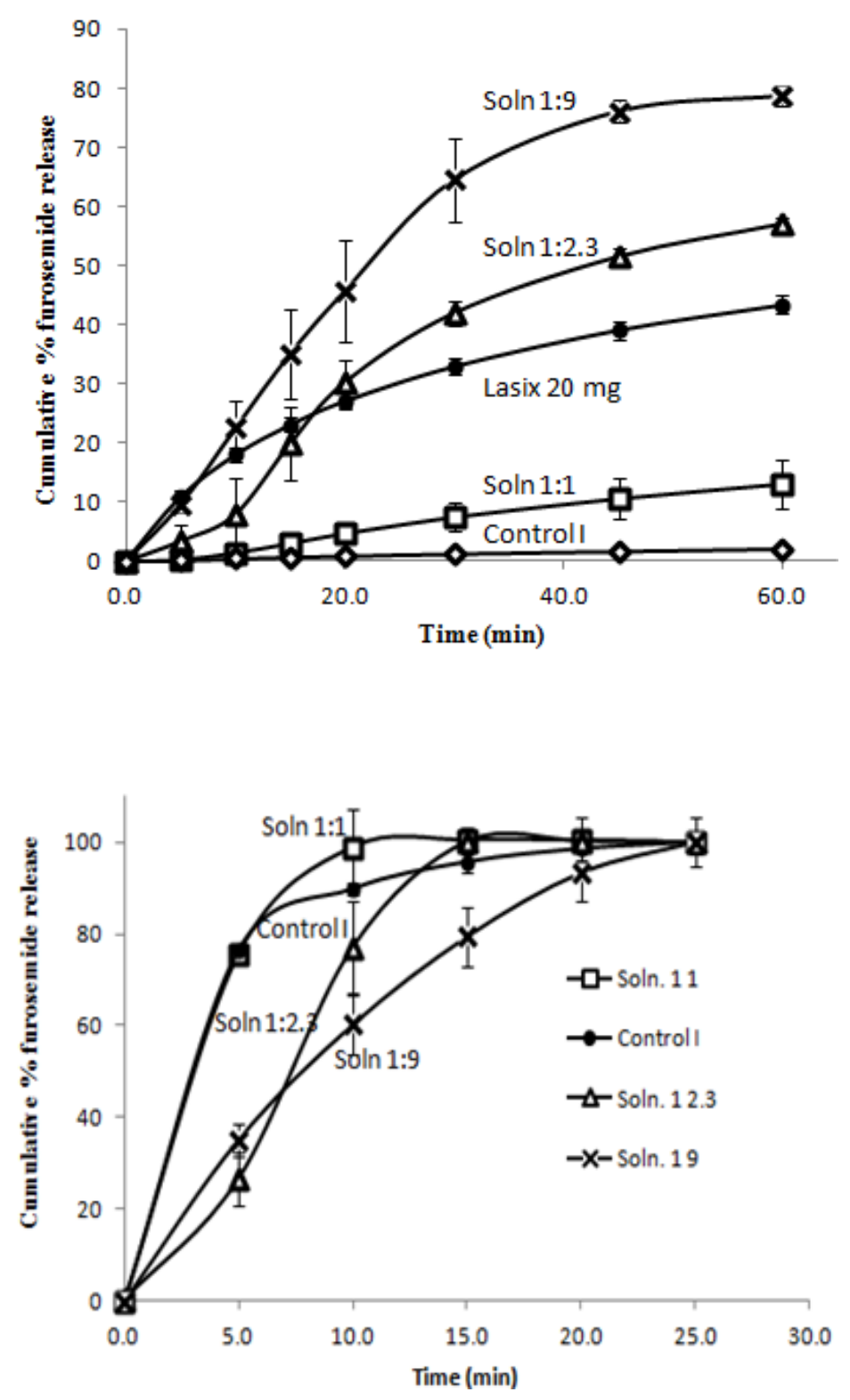

Figure 3-22. Dissolution profile of formulation II in SGF (pH 1.2) and phosphate buffer (pH 5.8).

Soln. SD 1:1 amorphous solid dispersion with furosemide to OSA starch $=1: 1(\mathrm{w} / \mathrm{w})$; Soln. SD 1:2.3 amorphous solid dispersion with furosemide to OSA starch $=1: 2.3(\mathrm{w} / \mathrm{w})$; Soln. SD 1:9 amorphous solid dispersion with furosemide to OSA starch $=1: 9(\mathrm{w} / \mathrm{w})$; Control I was amorphous furosemide ammonia salt; Lasix $20 \mathrm{mg}$ was commercial furosemide tablet, the strength was $20 \mathrm{mg} /$ tablet. 


\section{CHAPTER 4. PHASE SOLUBILITY OF FUROSEMIDE WITH HP-B-CD AND SBE-B-CD, PREPARATION AND CHARACTERIZATION OF SOLID DISPERSIONS OF FUROSEMIDE AND SBE-B-CD}

The purpose of the present study was to characterize the phase solubility of furosemide with Hydropropyl- $\beta$-Cyclodextrin (HP- $\beta-\mathrm{CD})$ and Sulfobutylether- $\beta$ Cyclodextrin (SBE- $\beta-C D$ ); solid disperson of furosemide and SBE- $\beta-C D$ prepared by kneading, spray drying and freeze drying methods. The measured aqueous solubility of furosemide was $96 \mu \mathrm{g} / \mathrm{ml}$. The phase solubility of furosemide-HP- $\beta$-CD complex was increased from 1.3-4.1 fold respectively in the concentration of 10-100 mM HP- $\beta-C D$. The K 1:1 and CE of the complex was $81 \mathrm{M}^{-1}$ and 0.01 respectively. While phase solubility of furosemide-SBE- $\beta$-CD complex was increased from 3.6-30.6 fold respectively in 10-100 mM of cyclodextrins. The K 1:1 and CE of the complexes was 269 $\mathrm{M}^{-1}$ and 0.0318 respectively. The equilibrium solubility and drug loading of solid dispersions were $167.9 \pm 3.04 \mu \mathrm{g} / \mathrm{ml}, 165.5 \pm 7.02 \mu \mathrm{g} / \mathrm{ml}, 115.0 \pm 4.04 \mu \mathrm{g} / \mathrm{ml}, 86.0 \pm$ $19.88 \mu \mathrm{g} / \mathrm{ml}$ and $12.9 \pm 0.23 \%, 14.7 \pm 0.33 \%, 13.6 \pm 0.14 \%, 7.04 \pm 0.72 \%$ respectively for spray drying, freeze drying, kneading method and physical mixture ( drug: SBE- $\beta-C D$ 1: $1 \mathrm{~mol} / \mathrm{mol})$. For drug to SBE- $\beta$-CD $(1: 2 \mathrm{~mol} / \mathrm{mol})$ for spray drying and freeze drying solid dispersions, the equilibrium solubility was $162.6 \pm 19.8 \mu \mathrm{g} / \mathrm{ml}$ and $163.0 \pm 5.87 \mu \mathrm{g} / \mathrm{ml}$ respectively; the drug loading were $8.1 \pm 0.43 \%$ and $7.5 \pm 0.99 \%$. FTIR, SEM and DSC further verified the complex formation. XRPD showed that drug was in amorphous form in spray dried sample, while in other samples, drug was crystalline. The intrinsic dissolution data demonstrated that in SGF, the drug release was in the rank of SD $>\mathrm{KD}>$ FD $>$ PM, while in the phosphate buffer ( $\mathrm{pH} 5.8)$, the drug release was in the order of KD $>\mathrm{PM}>\mathrm{FD}>\mathrm{SD}$. Solid dispersion tablet dissolution showed in acidic medium, drug release was in the order of $\mathrm{SD}>\mathrm{FD}>\mathrm{KD}>\mathrm{PM}>$ commercial tablets, While in phosphate buffer ( $\mathrm{pH}$ 5.8), drug released completely in 15 min for all samples.

\subsection{Introduction}

Cyclodextrin is cyclic oligomer of glucose which was extracted and separated from starch. It was widely used as drug solubilizer to enhance the aqueous solubility of poor water soluble drugs. The shape of cyclodextrin is like a double layer cylinder. The outside layer is hydrophilic which forms hydrogen bonds with water molecules, the inside cavity is hydrophobic which interacts with hydrophobic molecules or hydrophobic moiety of compound [114]. The molecular complex formation is the major mechanism for cyclodextrin solubilization function. Commonly, $\alpha, \beta$ or $\gamma$-cyclodextrin are often used which include 6,7 or 8 glucose units. Besides original cyclodextrin, modified cyclodextrins which include HP- $\beta-C D$ and SBE- $\beta-C D$ are also used in many commercial products. The administration route is various, such as oral, rectal, buccal, ophthalmic, intravenous and intramuscular [115]. Compared to the original cyclodextrins, the modified cyclodextrins have benefits, such as low toxicity, high water solubility and high solubilization capacity [116]. 
Furosemide is a loop diuretic which acts on the kidney to remove excess water and electrolytes into urine, thus reducing the body fluid. Furosemide was used to treat hypertension, congestive heart failure and edema [117]. It has also been used as veterinary medicine to treat horse bleeding [118]. Current drug products include tablets, oral solution and i.v. solution. The published oral bioavailability (BA) of furosemide was various, from $60 \sim 70 \%$ [119], or around 50\% [120], or in the range $37 \sim 51 \%$ [114, 121]. Its oral absorption was quick, peak serum concentration $\left(\mathrm{C}_{\max }\right)$ was between $60 \sim 90 \mathrm{~min}$ [122]. A big intra-viability around $20 \sim 84 \%$ and inter- subjects viability around $20 \sim 61 \%$ of BA were reported, too [98]. Furosemide was a BCS class IV drug which was low solubility and low permeability [123]. Chungi et al. investigated the site-specific absorption of furosemide in GI tract in rat model: the drug was administered to stomach and small intestine, the rapid absorption occurred when furosemide was administered to stomach [124]. Site-specific absorption was also studied in human, an oral immediate

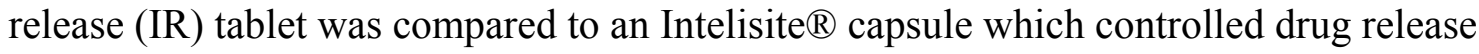
at a specific site in GI tract. In the clinical study, the relative BA of Intelisite ${ }^{\circledR}$ capsule is $71 \%$ to oral IR tablet when furesemide was released at proximal small intestine, and there is no absorption when drug release at colon [125]. Furosemide dissolution in the stomach and then rapidly absorbed from upper GI tract, dissolution and solubility in stomach is the rate limiting step for its oral absorption [115, 122, 123].

Furosemide is one of the few oral BCS class IV drug products. It is often selected as model drug to test new formulation techniques or as candidate for drug optimization since its low and various BA. Cyclodextrins including $\beta$-cyclodextrin (CD), dimethyl- $\beta$ $\mathrm{CD}$ and random-methylated $\beta-\mathrm{CD}$ were investigated for use in furosemide formulations. The solubility of furosemide is increased 3, 137 and 75 fold for $\beta C D$, dimethyl- $\beta$-CD and random-methylated $\beta-\mathrm{CD}$ respectively when the concentration of cyclodextrin is $300 \mathrm{mM}$ [126]. The mechanism of complexation of furosemide and HPBCD as well as SBEBCD are characterized using DSC, XRPD and NMR by Spamer et, al.: the characteristic melting combined a decomposition peak shifted to higher temperature range in DSC; the XRPD spectrums show that the degree of crystallinity is reduced; ${ }^{1} \mathrm{HNMR}$ and 2D-NMR (ROESY) prove that the furan ring and part of the aromatic ring are entrapped in the hydrophobic cavity of cyclodextrin [100]. Phase solubility, dissolution properties and permeability of furosemide and HP- $\beta-C D$ complex were also studied [119, 127, 128].

Various techniques have been used in furosemide solid formulation to increase its aqueous solubility and dissolution rate, i.e. dendrimer complex [129], the solubility increases around $110 \%$ per $\mathrm{mM}$ dendrimer added at the buffer with $\mathrm{pH} 4$ to 6 . The furosemide concentration in dissolution media is around $8 \mu \mathrm{g} / \mathrm{ml}$ at 2 hour point and 15 $\mu \mathrm{g} / \mathrm{ml}$ at 12 hour point under $\mathrm{pH} 1.2$; while drug concentration is around $12 \mu \mathrm{g} / \mathrm{ml}$ at 2 hour point and $18 \mu \mathrm{g} / \mathrm{ml}$ at 12 hour point when dissolution medium is $\mathrm{pH} 5.8$.

Self emulsifier drug delivery system technique has also applied to furosemide formulations [130]: mixture of surfactant and oil is screened to form self-microemulsion core, then furosemide is loaded at $1-10 \%$. Solubility of furosemide in SEDDS in $1 \mathrm{mM}$ hydrochloric acid solution $(0.5 \mathrm{ml} \mathrm{SEDDS} / 250 \mathrm{ml})$ is increased to $5.31 \mathrm{mg} / \mathrm{ml}$. Drug 
dissolution results show that the fastest drug release is around $55 \%$ and $100 \%$ at 4 hour point in $900 \mathrm{ml}$ dissolution medium ( $\mathrm{pH} 3.0$ and $\mathrm{pH} 6.8$ ). The amount of drug in dissolution medium is not mentioned in article. Furosemide permeability increases as well in SEDDS.

In addition to above techniques, solid dispersion is applied in many furosemide formulations. At $75 \mathrm{~min}, 80 \%$ drug released in dissolution medium ( $\mathrm{pH} 5.5)$ from $2 \mathrm{mg}$ thermal carbonization mesoporous silicon (TCPSi) microparticles with about $40 \%$ drug loading. Furosemide permeation has been increased 5 fold when drug was loading on TCPSi microparticles [116, 131]. In furosemide-crospovidone coprecipitating or cogrinding samples, drug is present in amorphous form. At $60 \mathrm{~min}$, drug concentration in dissolution medium (SGF, pH1.2) reached $25 \mathrm{mg} / \mathrm{L}$ [12]. Makoto et al. investigated the spray dried furosemide-Eudragit RS100 and RL100 solid dispersion: drug was in amorphous form, the dissolution showed solid dispersion initial release completely in less than 1 hour, then over time until 24h, drug concentration in dissolution medium (pH6.8) decreased due to absorption effect of polymer resin. Solid dispersion with $25 \%$ drug loading keeps its amorphous form for 24 days under $45^{\circ} \mathrm{C} / 75 \% \mathrm{RH}$ [132]. Shin et al. used TPGS to make solid dispersions, and the drug was in the amorphous form. At around $30 \%(\mathrm{w} / \mathrm{w})$ drug loading, the released concentration of furosemide was $40 \mathrm{mg} / \mathrm{L}$ in SGF $(\mathrm{pH} 1.2)$ [12]. Another two hydrophilic polymers: HPMC E50 LV and urea were studied to make solid dispersion. At 1 hour, around $70 \%$ and $35 \%$ drug released from solid dispersions: API to HPMC E50 LV and urea were 1:2 and 1:5 respectively. Dosage forms equal to $10 \mathrm{mg}$ drug was tested in $900 \mathrm{ml} \mathrm{SGF}$ (pH 1.2) [133].

Changing solubility and dissolution via solid dispersion, other formulation techniques including control release from EVA and PEG400 matrix tablet [134], tastemasking [135], multiple unit gastroretentive floating drug delivery systems [136], solubilization using micellar solutions of ethylene oxide and styrene oxide diblock copolymers [137] were all applied to furosemide.

In the present study, the author selected HPBCD and SBEBCD for phase solubility experiment, and then SBEBCD was further used as carrier to make furosemideSBEBCD solid dispersion by three methods. The phase solubility, solid dispersion of furosemide and SBEBCD including aqueous solubility, dissolution (solid dispersion and tablet), and fully characterization (XRPD, DSC, FTIR, DVS), physical stability etc. are presented first time.

\subsection{Material and Methods}

\subsubsection{Material}

Furosemide (Micronized) was generously donated by TEVA-TECH (Petah Tiova, 49131, Israel). Hydropropyl - $\beta$-cyclodextrin (HP- $\beta$-CD) was provided by Roquette America, Inc. Sulfobutylether- $\beta$-cyclodextrin (SBE- $\beta$-CD Captisol ${ }^{\circledR}$ ) was bought from 
Cydex Inc. (Lenexa, Kansas, USA). All other chemicals were reagent grade and used as received.

\subsubsection{Phase solubility, apparent stability constant, complex efficiency}

The phase solubility test was performed as Higuchi and Connors method [138]. Excess amount of drug was added into triplicate $20 \mathrm{ml}$ screw capped vials which contained $10 \mathrm{ml}$ various concentrations of HP- $\beta-\mathrm{CD}$, SBE- $\beta-\mathrm{CD}$ solution and R.O. water (pH 5.9). The vials were vortexed for 30 seconds at $1000 \mathrm{rpm}$, and then put on a shaker, shaking at room temperature $\left(25 \pm 1^{\circ} \mathrm{C}\right)$ for three days. At the end of each day, the sample suspension was filtered through a $0.45 \mathrm{um}$ membrane filter and then diluted to proper concentration for HPLC analysis. One way ANOVA was used to test time needed for reaching equilibrium solubility of each complex. The HPLC system was a Shimadzu LC10 AD together with a Nova-Pak ${ }^{\circledR C} 18$ column (Waters, $4 \mu \mathrm{m}, 150 \mathrm{~mm}$ X $3.9 \mathrm{~mm}$, i.d.) and Diode Array Detector (Model SPD-M 10A). The mobile phase was $10 \mathrm{mM}$ potassium phosphate monobasic and methanol $(60 / 40, \mathrm{v} / \mathrm{v})$, adjusted $\mathrm{pH}$ to 2.5 by phosphoric acid. The flow rate was $1 \mathrm{ml} / \mathrm{min}$ and the detective wavelength was $276 \mathrm{~nm}$.

The complexation constant $\left(\mathrm{K}_{1: 1}\right)$ and complex efficiency (CE) were calculated according to the Equations 4-1, 4-2 and the slope from phase solubility diagram.

$$
\begin{gathered}
\mathrm{K}_{1: 1}=\text { slope } / \mathrm{S}_{0} \text { (1-slope) }\left(\mathrm{S}_{0} \text { was solubility in water }\right) \\
\mathrm{CE}=\mathrm{S}_{0} \cdot \mathrm{K}_{1: 1}=\text { slope } /(1 \text { - slope })
\end{gathered}
$$

\subsubsection{Preparation of furosemide: SBE- $\beta$-CD solid dispersion}

\subsubsection{Kneading (KD)}

A kneader (Model IKA Duplex HKD 06D, Germany) was used to prepare furosemide loaded SBE- $\beta$-CD $(1: 1 \mathrm{~mol} / \mathrm{mol})$ solid dispersion. The powder mixture of furosemide $20 \mathrm{~g}$ and SBE- $\beta-C D$ 130.6g was wetted by ethanol and water $85: 15(\mathrm{v} / \mathrm{v})$ to form paste and then was kneaded for $35 \mathrm{~min}$. The paste was dried overnight at $60^{\circ} \mathrm{C}$ and then ground into powder by using coffee grinder.

\subsubsection{Freeze dry (FD)}

Furosemide and SBE- $\beta$-CD 1g, 6.53g or $13.06 \mathrm{~g}(1: 1$ and $1: 2 \mathrm{~mol} / \mathrm{mol})$ were dissolved in acetone / water $(1: 1 \mathrm{v} / \mathrm{v})$ separately, and then mixed the two solutions to obtain a clear sample solution. The sample solution was frozen before putting in a freeze dryer (Labconco 4.5 liter freeze drying system) and then solvent evaporated under vacuum to get dry powder. 


\subsubsection{Spray drying (SD) and physical mixture}

The sample solution was prepared as that in freeze dryer and then delivered to the nozzle $(0.7 \mathrm{~mm}$ i.d.) of a Buchi 190 mini spray dryer (Switzerland) at a flow rate 5 $\mathrm{ml} / \mathrm{min}$. The inlet temperature was $70{ }^{\circ} \mathrm{C}$ and outlet temperature was $41-44^{\circ} \mathrm{C}$; the compression air flow was about $700 \mathrm{~L} / \mathrm{h}$ and aspirator was $80 \%\left(33 \mathrm{~m}^{3} / \mathrm{h}\right)$. Physical mixture of furosemide and SBE- $\beta-\mathrm{CD}(1: 1 \mathrm{~mol} / \mathrm{mol})$ was well mixed by passing $60 \mathrm{mesh}$ sieve at multiple times.

\subsubsection{Aqueous solubility of solid dispersion in water}

The solid dispersion aqueous solubility was measured as in the method in 2.2 . Excess amount of solid dispersion powder was added to triplicate $20 \mathrm{ml}$ screw capped vial which contains $10 \mathrm{ml} \mathrm{R.O.} \mathrm{water} \mathrm{(pH} \mathrm{6.0).} \mathrm{The} \mathrm{vials} \mathrm{were} \mathrm{vortexed} \mathrm{for} 30$ seconds at $1000 \mathrm{rpm}$, and then put on a shaker, shaking at room temperature $\left(25 \pm 1^{\circ} \mathrm{C}\right)$ for three days. At the end of each day, the sample suspension was filtered through $0.45 \mu \mathrm{m}$ membrane filter and then diluted to proper concentration for HPLC analysis.

\subsubsection{Solid dispersion powder characterization}

Particle size distribution (PSD), surface area, true density and bulk density were measured for drug, SBE- $\beta-C D$, physical mixture and solid dispersions. A static laser scattering particle size analyzer (Saturn DigiSizer 5200, Micromeritics ${ }^{\circledR}$ ) was used for PSD measurements. A furosemide saturated isopropyl alcohol solution was prepared to disperse sample powder. The experiments were performed in triplicate. The surface area was measured with Gemini surface area analyzer (Micromeritics ${ }^{\circledR}$ ) and calculated according to BET theory by the instrument automatically. True densities were determined using a pycnometer (Micromeritics ${ }^{\circledR}$ AccuPyc 1330) at ambient temperature. The bulk densities were assessed using around $8 \mathrm{ml}$ sample powder in a $10 \mathrm{ml}$ graduated cylinder.

\subsubsection{Shape and surface morphology}

The photomicrographs of Furosemide, SBE- $\beta-C D$, physical mixture and three solid dispersion powders were obtained by environmental scanning electron microscope (ESEM) with backscattered electrons detector (QUANTA FEG 200, FEI Company). Powders were mounted on a double faced adhesive carbon tape, low vacuum (50 and 80 $\mathrm{Pa}$ ) applied in the chamber allows us to observe the powders without further treatment. The voltage was $10 \mathrm{kV}$, working distance was 10 to $8 \mathrm{~mm}$, spot size was 3 and the magnification time was from $250 \mathrm{X}$ to $5000 \mathrm{X}$. 


\subsubsection{Drug content in solid dispersion powder}

Exactly weighed amounts of solid dispersion powders triplicate in the molar ratio of $1: 1$ of furosemide to SBE- $\beta-C D$ were dissolved in the mobile phase and then sonicated samples for $10 \mathrm{~min}$. Then the drug content was determined by HPLC after dilute to appropriated concentration.

\subsubsection{Spectroscopic and thermal characteristics}

Fourier transform-infrared spectra were obtained on a FT-IR spectrometer (FT-IR Perkin Elmer 100 UTAR). The sample powder was measured directly and no additional preparation. X-ray powder diffraction (XRPD) was applied to study the solid state form of solid dispersions. Thermal characteristics of furosemide, SBE- $\beta-C D, P M$, and solid dispersions were obtained by a differential scanning calorimeter (DSC100, Texas Instrument, USA). The samples were placed in aluminum pans and were tested with the following parameters: equilibrated at $30^{\circ} \mathrm{C}$, ramp temperature $10^{\circ} \mathrm{C} / \mathrm{min}$ up to $310{ }^{\circ} \mathrm{C}$ for SBE- $\beta$-CD, ramp temperature $5{ }^{\circ} \mathrm{C} / \min$ up to $240{ }^{\circ} \mathrm{C}$ for furosemide and solid dispersions.

\subsubsection{Dissolution}

\subsubsection{Intrinsic dissolution}

Intrinsic dissolution behavior of furosemide and SBE- $\beta$-CD binary system was conducted to see if process methods had influence on drug release. Solid dispersion powder which was equal to $5 \mathrm{mg}$ or $20 \mathrm{mg}$ furosemide for SGF (pH 1.2) or phosphate buffer (pH 5.8) was compressed into intrinsic dissolution disks (USP Wood apparatus) with surface area $0.5 \mathrm{~cm}^{2}$ using a Carver press (Carver Inc., IL, USA ). The compress force was $1.1 \mathrm{mTon}$, dwelling time was 3 seconds. The dissolution medium was $600 \mathrm{ml}$, paddle speed was set as 100rpm and other parameters and test method were same as 2.6.1.

\subsubsection{Tablet dissolution}

Simulated gastric fluid ( $\mathrm{pH}$ 1.2) and phosphate buffer ( $\mathrm{pH}$ 5.8) were prepared for tablet dissolution test. The experiment was conducted at $37^{\circ} \mathrm{C}$ using USP II method with 50rpm paddle stirring speed and the volume of medium was $900 \mathrm{ml}$. Three solid dispersions and physical mixture equivalent to $20 \mathrm{mg}$ furosemide plus $4.7 \% \mathrm{Ai}-\mathrm{Di}$-Sol were compressed into tablets. Compress force was $1.1 \mathrm{mTon}$, dwelling time was 3 seconds. The percentage drug release was monitored using an on-line UV-Fiber optic dissolution system (Dynamic Dissolution Monitor ${ }^{\mathrm{TM}}$ system, Woodbridge Scientific LLC, CT, USA). 


\subsubsection{Dynamic vapor sorption (DVS)}

The hygroscopicity of API, physical mixture and solid dispersions were analyzed using a DVS-intrinsic instrument (Surface Measurement System) at $25^{\circ} \mathrm{C}$ over the humidity range $0 \%$ to $90 \% \mathrm{RH}$ with nitrogen gas as the carrier gas. Sample size of approximately $5 \mathrm{mg}$ was used according to the moisture uptake properties of SBE- $\beta-\mathrm{CD}$. The solid dispersion sample has same drug content.

\subsection{Results and Discussion}

\subsubsection{Phase solubility analysis}

The phase-solubility diagrams (PSD) of furosemide with SBE- $\beta-C D$ and HP- $\beta$ $\mathrm{CD}$ in water were showed in Figure 4-1, with the corresponding complexation constant $(\mathrm{Kc})$ and complex efficiency (CE) values calculated from each PSD (Table 4-1). The measured intrinsic solubility $\left(\mathrm{S}_{0}\right)$ and aqueous solubility were shown in Table 4-2. The aqueous solubility increased linearly as the function of SBE- $\beta-C D$ and HP- $\beta-C D$ concentration and the PSD can be classified as the $A_{L}$ type. Solubility of three day's complex were measured and compared using one way ANOVA. Furosemide: HP- $\beta-C D$ complex needed three days to reach equilibrium state at $100 \mathrm{mM}$, but for other lower concentrations and SBE- $\beta-C D$, one day is enough to reach equilibrium solubility. Since in many cases, the intrinsic solubility $\left(\mathrm{S}_{0}\right)$ estimated from PSD can be sensitive to errors, thus the $\mathrm{CE}$ become more frequently and convenient used for compare when selecting $\mathrm{CD}$ in formulation development.

\subsubsection{Aqueous solubility of solid dispersion in water}

From the phase solubility study, the SBE- $\beta$-CD showed better solubility enhancement effect. Thus, SBE- $\beta-C D$ was selected as carrier in the preparation of furosemide-loaded solid dispersions. In the present study, spray drying, freeze dry and kneader methods were chosen for sample prepare. The API and SBE- $\beta$-CD ratio was $1: 1$ and 1:2 (mol/mol) for spray drying and freeze drying, but for kneader method, only $1: 1$ ratio was tested considering the large amount of SBE- $\beta-C D$ consumed. The aqueous solubility was showed in Table 4-3. Freeze dry and spray drying methods gave better solubility enhancement effect on API, but with the increasing amount of SBE- $\beta$-CD in solid dispersion, the solubility didn't increase more which may further verify that furosemide and SBE- $\beta-C D$ formed 1:1 complex.

\subsubsection{Solid dispersion powder characterization}

The micromeritical characteristics, including particle size distribution (PSD), specific surface area, true density and bulk density of three methods prepared solid 


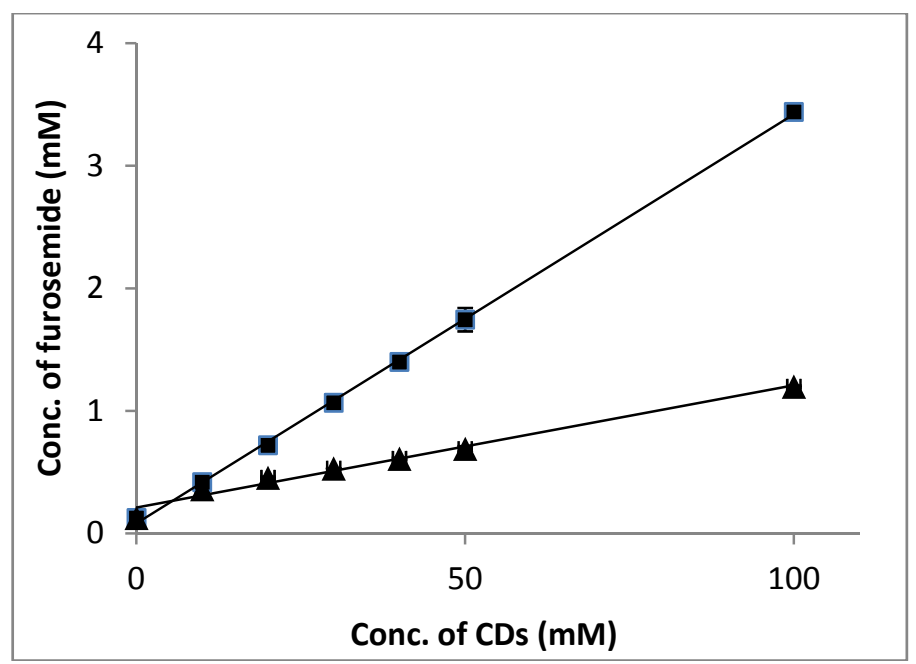

Figure 4-1. Phase-solubility diagrams of furosemide.

- Furosemide SBE- $\beta$-CD complex.

$\Delta$ Furosemide HP- $\beta-C D$ complex.

Table 4-1. K 1:1 and CE of phase-solubility studies of furosemide with SBE- $\beta$ CD and HP- $\beta-C D$.

\begin{tabular}{ccc}
\hline Excipient & $\mathbf{K}_{1: 1}\left(\mathbf{M}^{-1}\right)$ & $\mathbf{C E}$ \\
\hline SBE- $\beta-C D$ & 273 & 0.035 \\
HP- $\beta-C D$ & 81 & 0.010 \\
\hline
\end{tabular}

Table 4-2. Aqueous solubility of furosemide-CDs complex at $25^{\circ} \mathrm{C}$.

\begin{tabular}{cccc}
\hline $\begin{array}{c}\text { SBE- } \boldsymbol{\beta}-\mathbf{C D} \\
\text { conc. }(\mathbf{m M})\end{array}$ & $\begin{array}{c}\text { Solubility of furosemide } \\
\mathbf{a t} \mathbf{2 5}{ }^{\circ} \mathbf{C}(\boldsymbol{\mu g} / \mathbf{m l})\end{array}$ & $\begin{array}{c}\text { HP- } \boldsymbol{\beta}-\mathbf{C D} \\
\text { conc. }(\mathbf{m M})\end{array}$ & $\begin{array}{c}\text { Solubility of furosemide } \\
\mathbf{a t} \mathbf{2 5} \mathbf{~}^{\circ} \mathbf{C}(\boldsymbol{\mu} \mathbf{g} / \mathbf{m l})\end{array}$ \\
\hline 0 & $41.82 \pm 0.8$ & 0 & $41.82 \pm 0.8$ \\
10 & $138.5 \pm 3.0$ & 10 & $119.7 \pm 2.0$ \\
20 & $238.0 \pm 5.8$ & 20 & $150.4 \pm 3.1$ \\
30 & $352.8 \pm 10.3$ & 30 & $175.3 \pm 1.0$ \\
40 & $462.9 \pm 10.0$ & 40 & $201.8 \pm 3.1$ \\
50 & $576.9 \pm 31.6$ & 50 & $228.5 \pm 7.4$ \\
100 & $1137.6 \pm 6.0$ & 100 & $396.5 \pm 2.8$ \\
\hline
\end{tabular}


Table 4-3. Solubility of furosemide in solid dispersion prepared with three methods and the ratios of total solubility to the intrinsic solubility $(n=3)$.

\begin{tabular}{|c|c|c|c|}
\hline $\begin{array}{l}\text { Preparation } \\
\text { method }\end{array}$ & $\begin{array}{c}\text { API: SBE- } \beta-C D \\
\text { (mol:mol) }\end{array}$ & $\begin{array}{l}\text { Solubility } \\
(\mu \mathrm{g} / \mathrm{ml})\end{array}$ & $\begin{array}{c}\text { Complex solubility/ } \\
\text { intrinsic solubility } \\
(\mathbf{S} / \mathbf{S 0})\end{array}$ \\
\hline Kneader & $1: 1$ & $115.02 \pm 4.04$ & 2.74 \\
\hline \multirow{2}{*}{ Freeze dry } & $1: 1$ & $165.50 \pm 7.02$ & 3.94 \\
\hline & $1: 2$ & $163.01 \pm 5.87$ & 3.88 \\
\hline \multirow{2}{*}{ Spray dry } & $1: 1$ & $167.85 \pm 3.04$ & 4.01 \\
\hline & $1: 2$ & $173.9 \pm 3.57$ & 4.15 \\
\hline $\begin{array}{c}\text { Physical } \\
\text { mixture }\end{array}$ & $1: 1$ & $96.9 \pm 5.71$ & 2.31 \\
\hline
\end{tabular}

dispersion powder were shown in Table 4-4. The PSD was in the order of PM $>$ kneader $>$ FD $>$ SD, the solid dispersion prepared using spray drying method gave smallest particle size, thus the biggest specific surface area and least dense powders. The kneader method gave bigger PSD, smaller specific surface area and dentist powders among the three methods.

\subsubsection{Shape and surface morphology}

SEM images of furosemide, SBE- $\beta-C D$, three solid dispersion powders and physical mixture were shown in Figure 4-2. Pure micronized furosemide was crystalline powder, with long irregular cylinder shape; the SBE- $\beta-C D$ was in spherical shape suggested it was prepared by spray drying and the particle size was identical with results shown in Table 4-4.

The SEM image of physical mixture pictured the fine micronized crystalline furosemide coated on the surface of SBE- $\beta-C D$.

For the solid dispersion powders, KD powders have larger particle size than that of SD and FD samples. The particles have holes; in these holes furosemide crystals were highlighted and the crystalline distribution was not average. On fractures of the particles, crystals are rare; but on certain surfaces of these fine particles, the furosemide crystals were assembled in aggregates.

For the FD powder, fine furosemide crystals were assembled in aggregates. While in other big blocks which showed a smooth surface on picture, it was hard to distinguish furosemide from cyclodextrin matrix.

The spray dried solid dispersion was really small, to a large majority, much smaller than $5 \mu \mathrm{m}$ which was identical with PSD measurements. It's difficult to see in these powders the crystals of furosemide. At a magnification of 10000X, we find a few 
Table 4-4. Particle size, specific surface area, true density and bulk density of solid dispersions, furosemide, and SBE- $\beta-C D$.

\begin{tabular}{|c|c|c|c|c|}
\hline Samples & $\begin{array}{c}\text { Particle size } \\
\text { (Median } \pm \mathrm{SD}, \\
\mu \mathrm{m})^{*}\end{array}$ & $\begin{array}{c}\text { Specific } \\
\text { surface area } \\
\mathbf{m}^{2} / \mathbf{g} \\
\end{array}$ & $\begin{array}{c}\text { True } \\
\text { density } \\
\left(\mathrm{g} / \mathrm{cm}^{3}\right)\end{array}$ & $\begin{array}{l}\text { Bulk density } \\
\quad\left(\mathrm{g} / \mathrm{cm}^{3}\right)^{*}\end{array}$ \\
\hline Furosemide & $5.04 \pm 0.19$ & 3.3294 & 0.8293 & $0.2636 \pm 0.00056$ \\
\hline SBE- $\beta-C D$ & $1293.67 \pm 79.3$ & 0.0864 & 0.6603 & $0.5400 \pm 0.0018$ \\
\hline Kneader $^{\wedge}$ & $90.44 \pm 2.72$ & 0.4176 & 0.7344 & $0.6087 \pm 0.0234$ \\
\hline Freeze dry ${ }^{\wedge}$ & $46.19 \pm 0.932$ & 0.684 & 0.7759 & $0.5999 \pm 0.01292$ \\
\hline 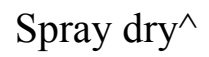 & $7.125 \pm 0.621$ & 1.3071 & 0.7814 & $0.3117 \pm 0.01161$ \\
\hline $\begin{array}{l}\text { Physical } \\
\text { miscure }^{\wedge}\end{array}$ & $123.82 \pm 2.54$ & 0.3894 & 0.6861 & 0.5494 \\
\hline
\end{tabular}

${ }^{*} \mathrm{n}=3$ for Particle size and bulk density.

$\wedge$ API to SBE- $\beta$-CD ratio is $1: 1$. 

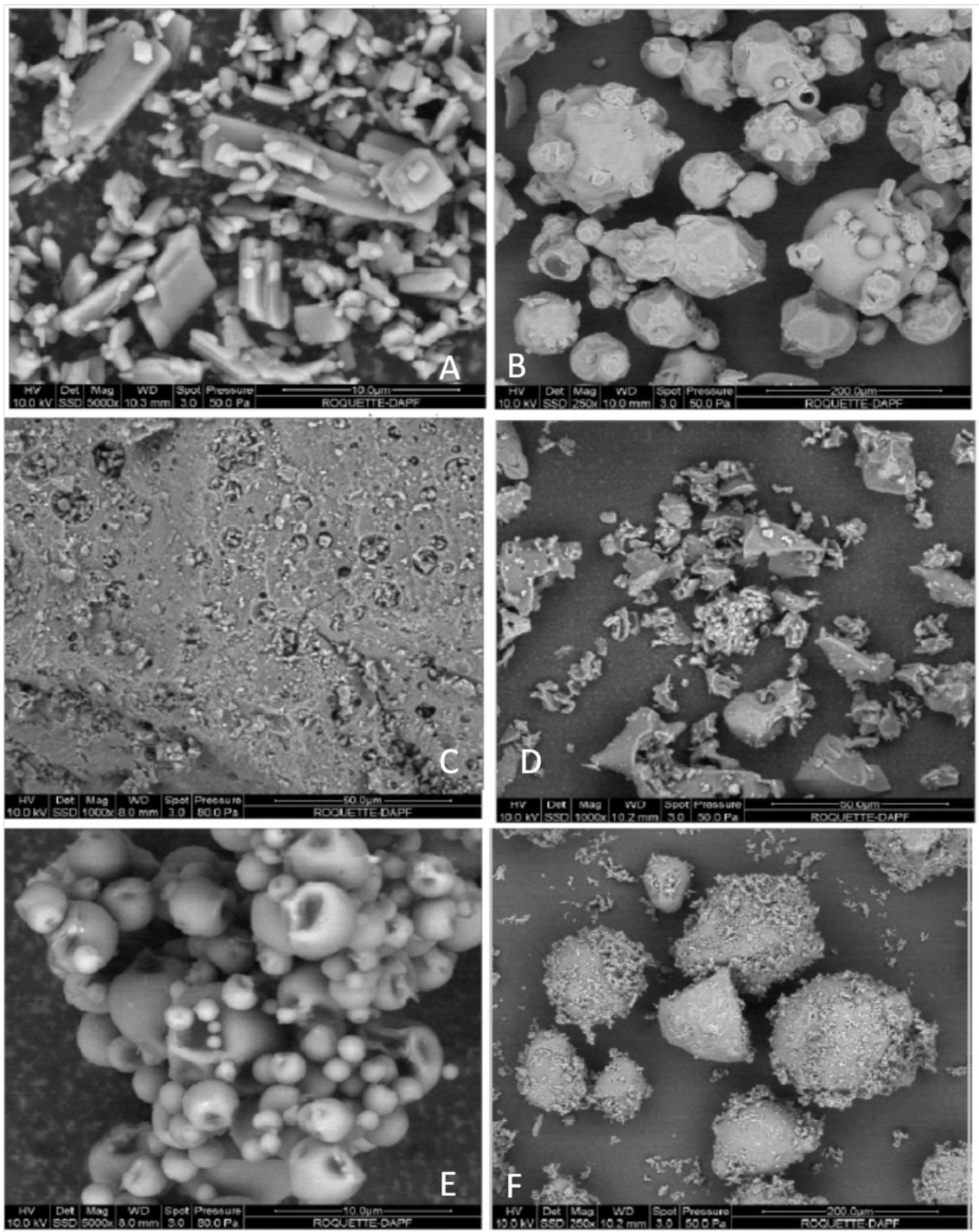

Figure 4-2. Scanning electron micrographs of furosemide, SBE- $\beta-C D$, and furosemide SBE- $\beta$-CD 1:1 sodid dispersion prepared by different methods.

(A)furosemide $(5000 \times)$, (B) SBE- $\beta-C D(250 \times)$, (C) solid dispersion prepared by the kneader method $(1000 \times)$, (D) solid dispersion prepared by the freeze drying method $(1000 \times),(E)$ solid dispersion prepared by the spray drying method $(5000 \times),(\mathrm{F})$ physical mixture $(250 \times)$. All solid dispersions were composed of furosemide : SBE- $\beta$-CD 1:1 (mol:mol). 
but they are discreet. Much of furosemide adhered to the periphery of the spray dried spheres of SBE- $\beta$-CD but a fraction remained free which was clear displayed in physical mixture powders.

\subsubsection{Drug content in solid dispersion powders}

The drug content in each solid dispersion sample was shown in Table 4-5. The ratio between furosemide and SBE- $\beta-C D$ was $1: 1(\mathrm{~mol}: \mathrm{mol})$. The results accorded with the theoretical content: $13.3 \%$ except in the physical mixture sample. The less drug content in physical mixture was caused by API loss in sieve mixing process: the hydrophobic API adhered on the metal screen and didn't dilute out by SBE- $\beta$-CD.

\subsubsection{Spectroscopic and thermal characteristics}

FTIR spectra of furosemide, SBE- $\beta$-CD and solid dispersions were presented in Figure 4-3. The characteristic absorption bands of furosemide at $1670 \mathrm{~cm}^{-1}$ was $C=O$ stretching vibration of carboxylic acid group, $1591 \mathrm{~cm}^{-1}$ and $1561 \mathrm{~cm}^{-1}$ were N-H bending , the $\mathrm{S}=\mathrm{O}$ stretching of the sulfonamide group was at $1318 \mathrm{~cm}^{-1}$ (asymmetric) and $1140 \mathrm{~cm}^{-1}$ (symmetric) [108]. The IR spectrum of physical mixture was similar with that of furosemide. In solid dispersios, the bands at $3399 \mathrm{~cm}^{-1}, 3350 \mathrm{~cm}^{-1}$ and $3282 \mathrm{~cm}^{-1}$ which were N-H stretching became wide and weak which was caused by bands overlap of SBE- $\beta-C D$ or amorphous furosemide. The $S=O$ stretching at $1140 \mathrm{~cm}^{-1}$ was shifted to $1153 \mathrm{~cm}^{-1}$ and $1670 \mathrm{~cm}^{-1} \mathrm{C}=\mathrm{O}$ stretching of carboxylic acid group shifted to $1175 \mathrm{~cm}^{-1}$ in spray dried sample indicated amorphous form of furosemide existed [117]. For KD and FD samples, the IR spectrum didn't change much compared to that of PM, API maintained its crystal form in these samples which was also in agreement with the results of XRPD (Figure 4-4).

The X-ray powder diffraction (XRPD) pattern of furosemide showed intense and sharp peaks in the spectrum proved it was crystalline [117]. The SBE- $\beta$-CD was amorphous without peak shown on XRPD. Samples prepared by kneading and freeze drying showed sharp peaks in the spectrum, but the intensity was not as high as that of API; the peaks indicated the crystalline form in the sample, since the drug content was around $13 \%$ in the two samples, the intensity of peaks was lower that of pure API. No distinguished peak shown in the spectrum of spray dried sample, the solid dispersion prepared by spray drying was predominantly amorphous form. A small crystal peak shown in the spectrum of physical mixture which indicated crystalline was here; and

Table 4-5. Drug content of solid dispersion samples.

\begin{tabular}{ccccc}
\hline API:CD=1:1 (mol:mol) & KD & FD & SD & PM \\
\hline Drug content $\%$ & $14.7 \pm 0.33$ & $13.6 \pm 0.15$ & $12.9 \pm 0.21$ & $7.1 \pm 0.17$ \\
\hline
\end{tabular}




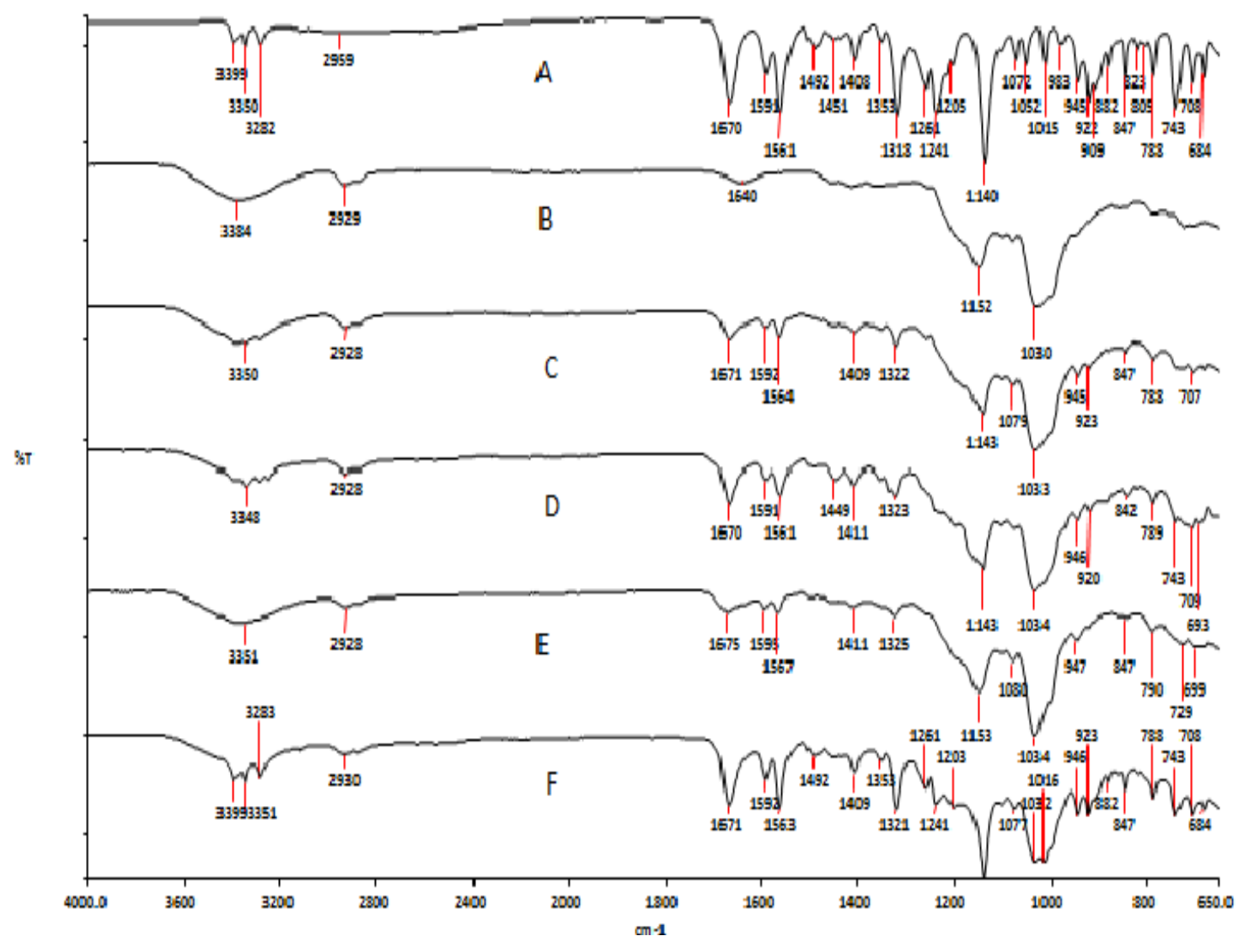

Figure 4-3. FTIR spectra of (A) furosemide (B) SBE- $\beta$-CD (C) KD solid dispersion (D) FD solid dispersion (E) SD solid dispersion and (F) physical mixture. 


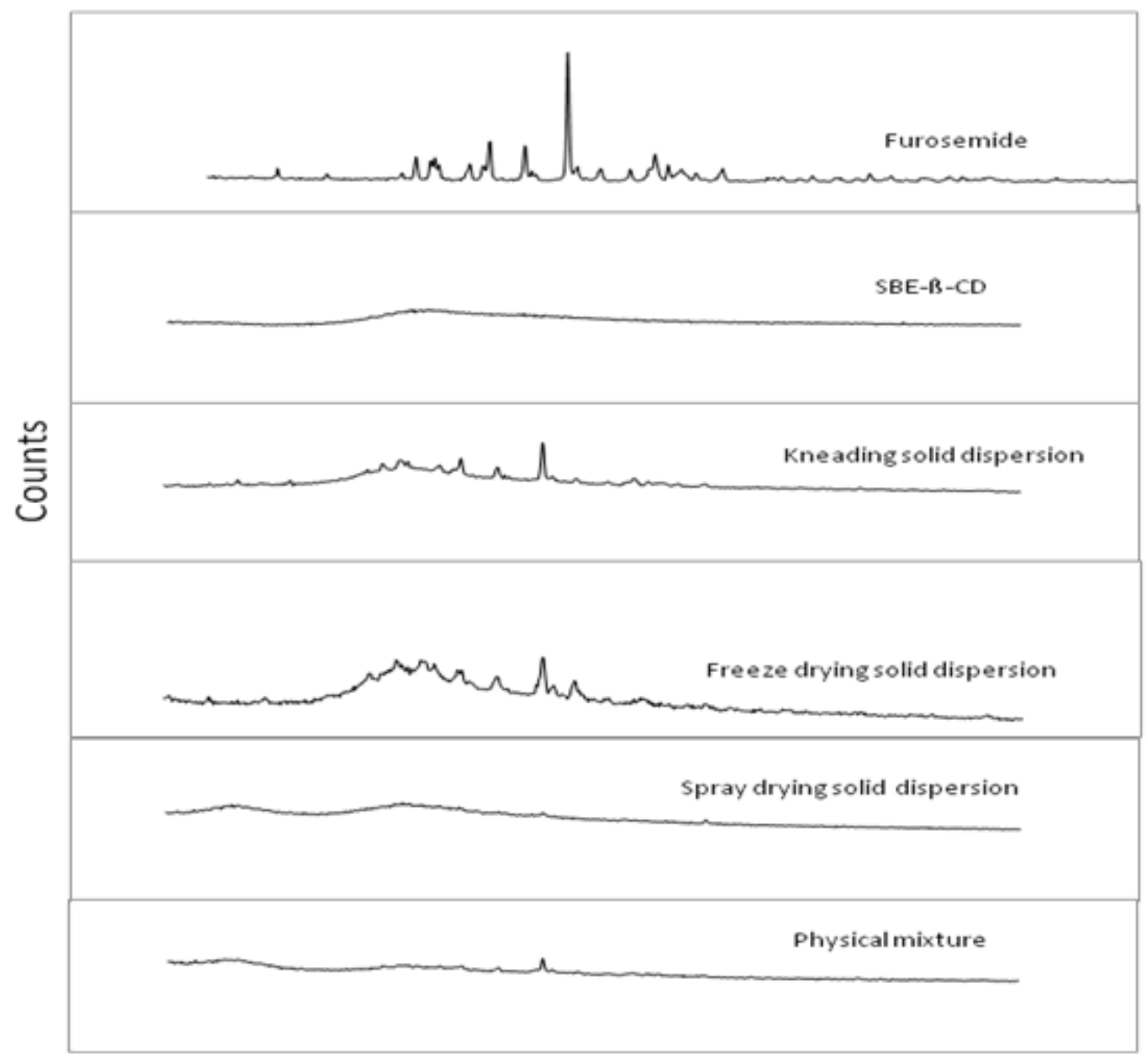

Angles $2 \Theta$

Figure 4-4. XRPD spectrums of furosemide, SBE- $\beta$-CD, furosemide solid dispersions prepared with kneader, freeze drying and spray drying method and physical mixture. 
low drug content (7\%) didn't incur sharp diffraction peaks. From the XRPD spectrum, the crystalline degree was in the order of API (crystal) $>$ Kneading $\approx$ Freeze drying $>$ physical mixture $>$ spray drying $>$ SBE- $\beta$-CD (amorphous).

The DSC spectrum was shown in Figure 4-5. In the temperature range 207 219 ${ }^{\circ} \mathrm{C}$, a sharp exothermic peak was the characteristic melting combined decomposition peak of furosemide. ${ }^{14}$ In kneading (KD), freeze drying (FD) and spray drying (SD) solid dispersion samples, this sharp peak disappeared and decomposition phenomena was postponed to higher temperature range: $223 \sim 230{ }^{\circ} \mathrm{C}$. No clear melting can be assigned to these spectrums. Actually, the author also tried the heat-cool-heat cycle and various heating rate $\left(20^{\circ} \mathrm{C}, 40^{\circ} \mathrm{C}\right.$ and $\left.100^{\circ} \mathrm{C} / \mathrm{min}\right)$ method to separate melting and decomposition events, but based on in house condition, no clear melting point was observed. For physical mixture (PM), the DSC was similar with that of other samples. All spectrums showed complex formed even in simple mixture.

\subsubsection{Dissolution}

\subsubsection{Intrinsic dissolution}

The intrinsic dissolution data (showed in Figure 4-6a and $\mathbf{b}$ ) demonstrated that in $\mathrm{SGF}$, the drug release was in the rank of $\mathrm{SD}>\mathrm{KD}>\mathrm{FD}>\mathrm{PM}$, while in the phosphate buffer ( $\mathrm{pH} 5.8$ ), the drug release was in the order of $\mathrm{KD}>\mathrm{PM}>\mathrm{FD}>\mathrm{SD}$. In acidic environment, the drug solubility was the limit factor for dissolution. The fastest drug release sample was amorphous spray dried sample. After compaction, small particles (more porosity) always form tighter compact and water penetration is slower than that of big particles. For similar crystalline samples, the dissolution rate of KD was faster than that of FD sample. In the phosphate buffer, dissolution rate was decided more by how fast the water penetrated into compact. Drug released faster from the loose compact KD and PM, but for SD and KD samples, a thin hydration layer formed on the surface of SD and FD sample which occluded continuing fast water penetration and made drug release slower after initial fast dissolution.

\subsubsection{Tablet dissolution}

After incorporating about 5\% disintegrant, solid dispersion powders were compressed into tablets. In acidic medium, drug release was in the order of SD $>$ FD $>$ $\mathrm{KD}>\mathrm{PM}>$ commercial tablets. This sequence was in accord with the solubility sequence. An abnormal phenomenon was observed that the spray dried and freeze dried tablet did not disintegrate which may caused by tight intra-particles physical binding. When drug released in phosphate buffer ( $\mathrm{pH} 5.8$ ), when solubility was not the limit factor, drug release finished in 15 mins for all samples. In this case, commercial product gave fastest drug release due to its perfect drug disintegration performance: disintegrated completely in 30 seconds (data is shown in Figure 4-7a and b). 


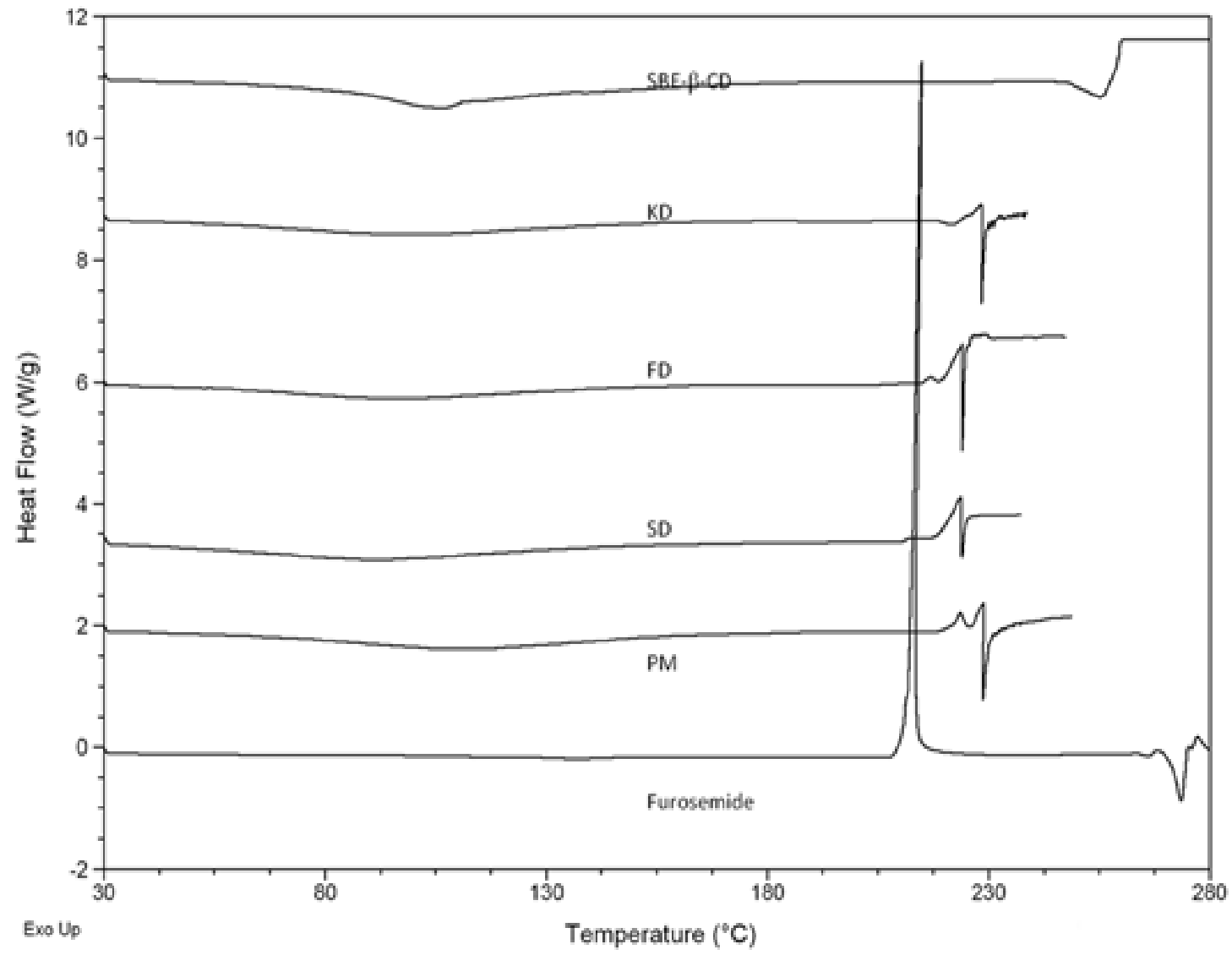

Figure 4-5. DSC spectrum of SBE- $\beta-C D$, solid dispersions and furosemide. The ratio of furosemide to SBE- $\beta-C D$ was $1: 1 \mathrm{~mol} / \mathrm{mol}$. 

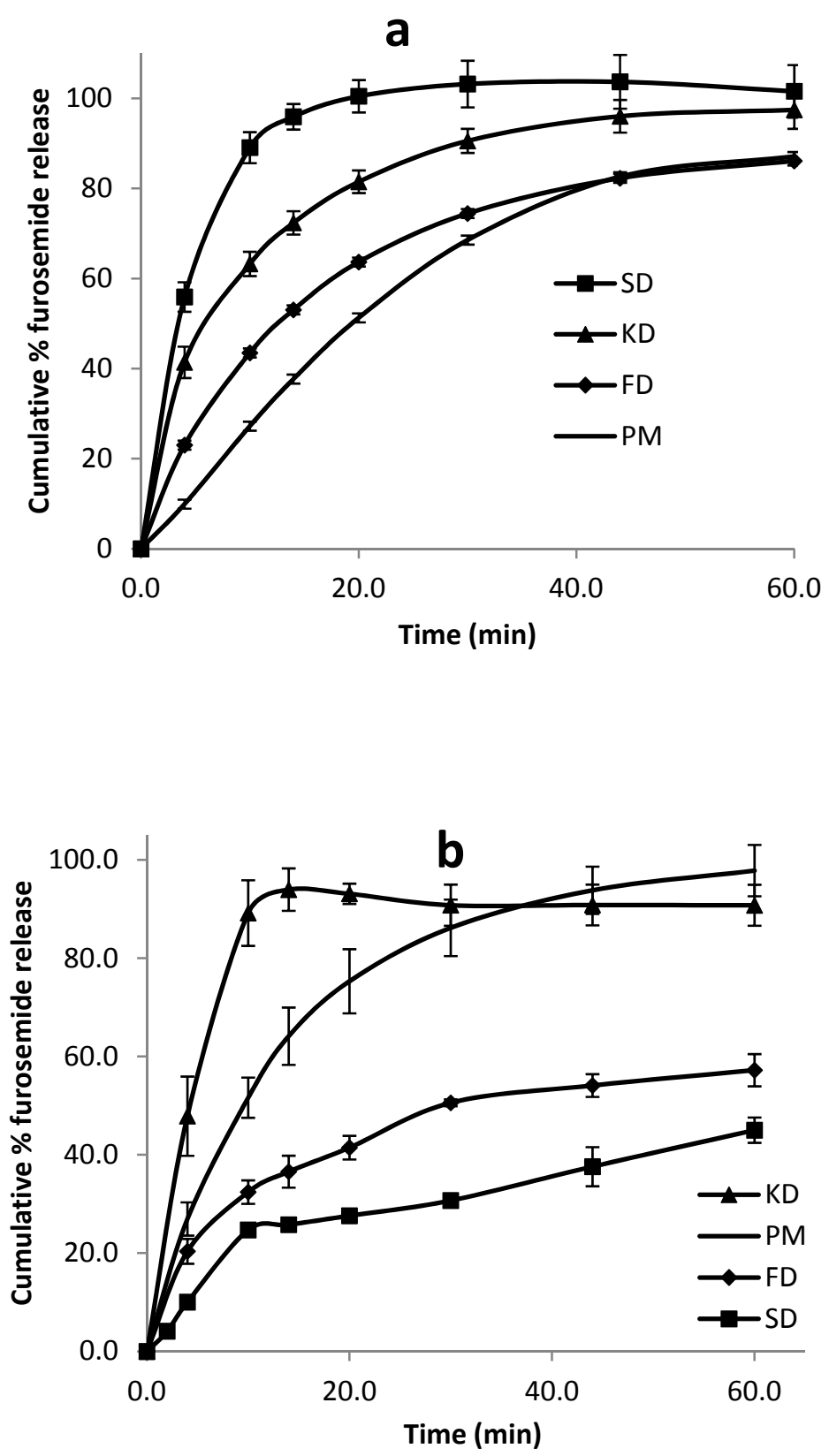

Figure 4-6. Solid dispersion intrinsic dissolution profile.

(a) Solid dispersion intrinsic dissolution in SGF ( $\mathrm{pH} 1.2)$; (b) Solid dispersion intrinsic dissolution in phosphate buffer ( $\mathrm{pH}$ 5.8). 

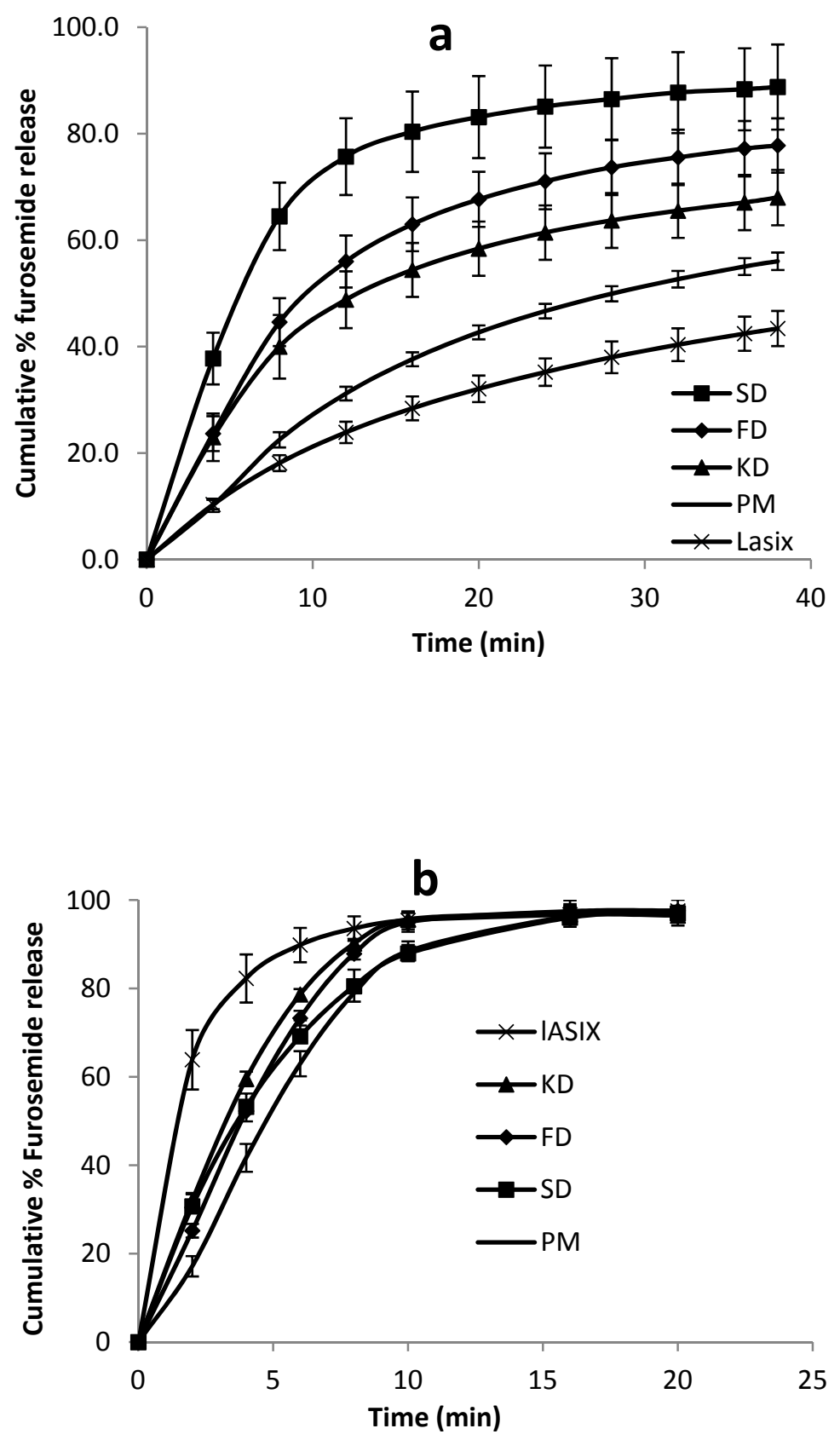

Figure 4-7. Solid dispersion tablet dissolution profile.

(a) Tablet dissolution in SGF ( $\mathrm{pH} 1.2$ ); (b) Tablet dissolution in phosphate buffer ( $\mathrm{pH}$ 5.8). 


\subsubsection{DVS}

Dynamic vapor sorption and desorption results were shown in Figure 4-8. The lower line with triangle marker was sorption curve and the upper line with square marker was desorption curve. Except for furosemide, the hydroscopicity of other samples was similar. The Low $\% \mathrm{RH}$ values resulted in low vapor sorption and the water was only adsorbed on the sample surface. At $20 \% \mathrm{RH}$, the weight gain was in the order of SD $(4.4 \%)>$ FD $(3.1 \%)>\mathrm{KD}(2.9 \%)>\mathrm{PM}(2.0 \%)>\mathrm{SBE}-\beta-\mathrm{CD}(1.6 \%)>\mathrm{API}(0.028 \%)$. This order was highly in accordance to the surface area of samples: the higher surface area, thehigh water uptake. In the case of API, the lowest water uptake was determined by its high hydrophobic properties. With increased RH, the sorption kinetics changed from dominated by surface adsorption processes to bulk absorption. At $80 \% \mathrm{RH}$, the water uptake order was SBE- $\beta$-CD $(35.3 \%)>\mathrm{SD}(33.7 \%)>\mathrm{PM}(32.5 \%)>\mathrm{KD}(30.8 \%)>\mathrm{FD}$ $(29.6 \%)>$ API $(0.1 \%)$. This bulk absorption was related to the amorphous property of samples which was indicated by SEM picture and XRPD spectrum as well. The high vapor sorption of SBE- $\beta-C D$ and SD sample are fully amorphous form.

\subsection{Conclusion}

The phase solubility of furosemide and SBE- $\beta-C D$ and dissolution of its solid dispersions were first presented. The anionic ion SBE- $\beta-\mathrm{CD}$ in water at acidic $\mathrm{pH}$ can interact with hydrogen ions which benefited the drug solubility in acidic environment besides the function of parent cyclodextrin. In spray dried solid dispersion, the drug was armorphous whereas in kneading and freeze dried samples, drug was crystal. When powders were compressed into tablets, the tight physical bonding in spray dried and freeze dried samples made it hard to disintegrate even with $4.7 \%$ disintegrant. For further applying spray dry techniques to prepare solid dispersions for compressing tablets, a granulation step would be needed to get proper flow and to lower interparticle bonding for better tablet disintegration. The drug release of Lasix ${ }^{\circledR}$ was faster than those of others at phosphate buffer but slower in SGF. This because the disintegrant in the formulation play a critical role for drug release when solubility was not limit-factor, while in SGF, the disintegrant didn't help drug solubilization. 

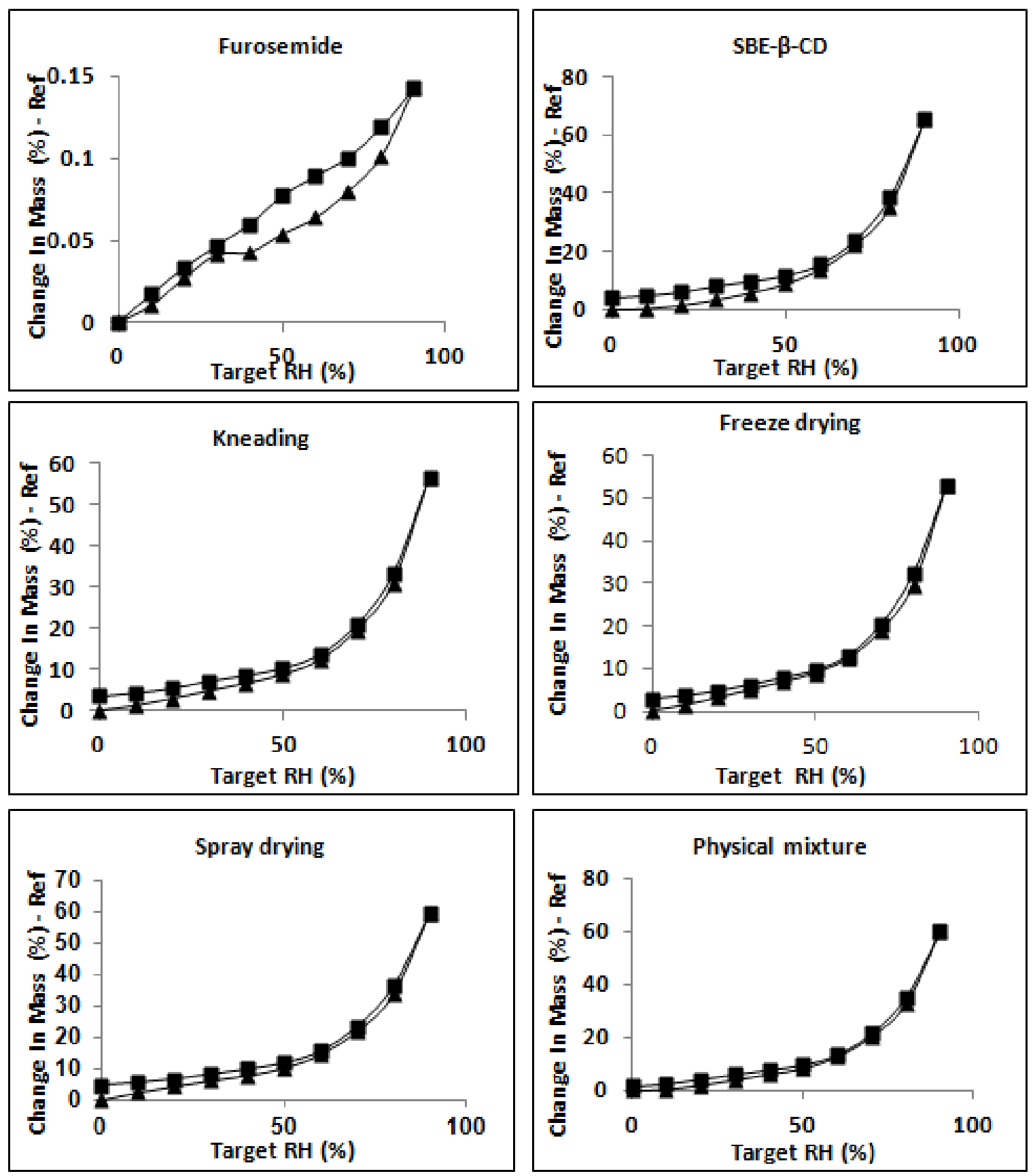

Figure 4-8. Dynamic sorption and desorption diagram of furosemide, SBE- $\beta-C D$, solid dispersions and physical mixture. 


\section{LIST OF REFERENCES}

1. Amidon, G.L., et al., A theoretical basis for a biopharmaceutic drug classification: the correlation of in vitro drug product dissolution and in vivo bioavailability. Pharmaceutical Research, 1995. 12(3): p. 413-20.

2. Lindahl, A., et al., Surface activity and concentration dependent intestinal permeability in the rat. Pharmaceutical Research, 1999. 16(1): p. 97-102.

3. Lobenberg, R. and Amidon, G.L., Modern bioavailability, bioequivalence and biopharmaceutics classification system. New scientific approaches to international regulatory standards. European Journal of Pharmaceutics and Biopharmaceutics, 2000. 50(1): p. 3-12.

4. Martinez, M.N. and Amidon, G.L., A mechanistic approach to understanding the factors affecting drug absorption: a review of fundamentals. Journal of Clinical Pharmacology, 2002. 42(6): p. 620-43.

5. Oh, D.M., Curl, R.L.and Amidon, G.L., Estimating the fraction dose absorbed from suspensions of poorly soluble compounds in humans: a mathematical model. Pharmaceutical Research, 1993. 10(2): p. 264-70.

6. Greenblatt, D.J., Smith, T.W.and Koch-Weser, J., Bioavailability of drugs: the digoxin dilemma. Clinical Pharmacokinetics, 1976. 1(1): p. 36-51.

7. Dressman, J.B., Amidon, G.L., and Fleisher,D., Absorption potential: estimating the fraction absorbed for orally administered compounds. Journal of Pharmaceutical Sciences, 1985. 74(5): p. 588-9.

8. Yamashita, S., et al., Optimized conditions for prediction of intestinal drug permeability using Caco-2 cells. European Journal of Pharmaceutical Sciences, 2000. 10(3): p. 195-204.

9. Cook, J., Addicks, W. and Wu, Y.H., Application of the biopharmaceutical classification system in clinical drug development--an industrial view. AAPS Journal, 2008. 10(2): p. 306-10.

10. Tukker, J.J., Oral Drug Absorption Prediction and Assessment, H.L. Jennifer B. Dressman, Editor, Marcel Dekker, Inc.: New York. p. 65.

11. Hubatsch, I., E.G. Ragnarsson, and P. Artursson, Determination of drug permeability and prediction of drug absorption in Caco-2 monolayers. Nature Protocol, 2007. 2(9): p. 2111-9. 
12. Sang-Chul Shin, I.-J.O., Yong-Bok Lee, Hoo-Kyun Choi, Jun-Shik Choi, Enhanced dissolution of furosemide by coprecipitating or cogrinding with crospovidone. International Journal of Pharmaceutics, 1998. 175: p. 17-24.

13. Tukker, J.J., Oral Drug Absorption Prediction and Assessment, H.L. Jennifer B. Dressman, Editor, Marcel Dekker, Inc.: New York. p. 79.

14. Guidance for Industry: Waiver of in vivo Bioavailability and Bioequivalence Studies for Immediate-Release Solid Oral Dosage Forms Based on a Biopharmaceutics Classification System in U.S. , F.D.A. Department of Health and Human Services, Center for Drug Evaluation and Research (CDER), Editor 2000 .

15. Martin's Physical Pharmacy \& Pharmaceutical Sciences 5th ed 2006: Lippincott Williams \& Wilkins.

16. Guidance for Industry: Immediate release Solid Dosage Forms. Scale-Up and Postapproval Changes. In: U.S., F.D.A. Department of Health and Human Services, Center for Drug Evaluation and Research (CDER), Editor 1995.

17. JB, D., Physiological aspects of the design of dissolution tests in Scientific Foundations for Regulating Drug Product Quality1997, Alexandria, VA: AAPS Press. 155-168.

18. Guidance for industry: immediate release solid oral dosage forms: scale-up and postapproval changes: chemistry, manufacturing, and controls, in vitro dissolution testing, and in vivo bioequivalence documentation, F.D. A., Editor 1995.

19. Duan, J.Z., K. Riviere, and P. Marroum, In vivo bioequivalence and in vitro similarity factor (f2) for dissolution profile comparisons of extended release formulations: how and when do they match? Pharmaceutical Research, 2011. 28(5): p. 1144-56.

20. Grabovac, V., et al., Papain: an effective permeation enhancer for orally administered low molecular weight heparin. Pharmaceutical Research, 2007. 24(5): p. 1001-6.

21. Bejugam, N.K., H.J. Parish, and G.N. Shankar, Influence of formulation factors on tablet formulations with liquid permeation enhancer using factorial design. AAPS PharmSci Technology, 2009. 10(4): p. 1437-43.

22. Whitehead, K., N. Karr, and S. Mitragotri, Safe and effective permeation enhancers for oral drug delivery. Pharmaceutical Research, 2008. 25(8): p. 17828. 
23. Patravale, V.B., A.A. Date, and R.M. Kulkarni, Nanosuspensions: a promising drug delivery strategy. Journal of Pharmacy and Pharmacology, 2004. 56(7): p. 827-40.

24. Shah, P., Use of Nanotechnologies for Drug Delivery. Materials Research Society Bulletin, 2006(31): p. 894-899.

25. Rabinow, B.E., Nanosuspensions in drug delivery. Nature Review Drug Discovery, 2004. 3(9): p. 785-96.

26. Konno, H. and L.S. Taylor, Influence of different polymers on the crystallization tendency of molecularly dispersed amorphous felodipine. Journal of Pharmaceutical Sciences, 2006. 95(12): p. 2692-705.

27. Grau, M.J., O. Kayser, and R.H. Muller, Nanosuspensions of poorly soluble drugs--reproducibility of small scale production. International Journal of Pharmaceutics, 2000. 196(2): p. 155-9.

28. Muller, R.H., C. Jacobs, and O. Kayser, Nanosuspensions as particulate drug formulations in therapy. Rationale for development and what we can expect for the future. Advance Drug Delivery Review, 2001. 47(1): p. 3-19.

29. Shi, H.G., et al., Characterization of crystalline drug nanoparticles using atomic force microscopy and complementary techniques. Pharmaceutical Research, 2003. 20(3): p. 479-84.

30. Akers, M.J., A.L. Fites, and R.L. Robison, Formulation design and development of parenteral suspensions. Journal of Parenteral Science Technology, 1987. 41(3): p. 88-96.

31. Holland, S. Special aspects of nanomedicines: Development, manufacturing and characterisations. in 1st international workshop on nanomedicines. 2010.

32. Pouton, C.W., Formulation of poorly water-soluble drugs for oral administration: physicochemical and physiological issues and the lipid formulation classification system. European Journal of Pharmaceutical Sciences, 2006. 29(3-4): p. 278-87.

33. Gershanik, T. and S. Benita, Self-dispersing lipid formulations for improving oral absorption of lipophilic drugs. European Journal of Pharmaceutics and Biopharmaceutics, 2000. 50(1): p. 179-88.

34. Jing-ling Tang, J.S.a.Z.-G.H., Self-Emulsifying Drug Delivery Systems: Strategy for Improving Oral Delivery of Poorly Soluble Drugs. Current Drug Therapy, 2007(2): p. 85-93. 
35. Tang, B., et al., Development of solid self-emulsifying drug delivery systems: preparation techniques and dosage forms. Drug Discovery Today, 2008. 13(1314): p. 606-12.

36. Singh, B., et al., Self-emulsifying drug delivery systems (SEDDS): formulation development, characterization, and applications. Critical Review in Therapeutic Drug Carrier Systems, 2009. 26(5): p. 427-521.

37. Gursoy, R.N. and S. Benita, Self-emulsifying drug delivery systems (SEDDS) for improved oral delivery of lipophilic drugs. Biomedine and Pharmacotherapy, 2004. 58(3): p. 173-82.

38. RAJANB MISTRY, N.S., A review: self emulsifying drug delivery system. International Journal of Pharmacy and Pharmaceutical Sciences, 2011. 3(Suppl 2): p. 23-28.

39. Davis, M.E. and M.E. Brewster, Cyclodextrin-based pharmaceutics: past, present and future. Nature Review Drug Discovery, 2004. 3(12): p. 1023-35.

40. Wikipedia. Cyclodextrin. Available from: http://en.wikipedia.org/wiki/Cyclodextrin. Accessed May 4th, 2012

41. Loftsson, T. and D. Duchene, Cyclodextrins and their pharmaceutical applications. International Journal of Pharmaceutics, 2007. 329(1-2): p. 1-11.

42. Chiou, W.L. and S. Riegelman, Pharmaceutical applications of solid dispersion systems. Journal of Pharmaceutical Sciences, 1971. 60(9): p. 1281-302.

43. Janssens, S. and G. Van den Mooter, Review: physical chemistry of solid dispersions. Journal of Pharmacy and Pharmacology, 2009. 61(12): p. 1571-86.

44. Qian, F., J. Huang, and M.A. Hussain, Drug-polymer solubility and miscibility: Stability consideration and practical challenges in amorphous solid dispersion development. Journal of Pharmaceutical Sciences, 2010. 99(7): p. 2941-7.

45. Walters, K.A., P.H. Dugard, and A.T. Florence, Non-ionic surfactants and gastric mucosal transport of paraquat. Journal of Pharmacy and Pharmacology, 1981. 33(4): p. 207-13.

46. Craig, D.Q., The mechanisms of drug release from solid dispersions in watersoluble polymers. International Journal of Pharmaceutics, 2002. 231(2): p. 13144 . 
47. Karavas, E., et al., Effect of hydrogen bonding interactions on the release mechanism of felodipine from nanodispersions with polyvinylpyrrolidone. European Journal of Pharmaceutics and Biopharmaceutics, 2006. 63(2): p. 10314.

48. Chen, Y., et al., Enhancing the bioavailability of ABT-963 using solid dispersion containing Pluronic F-68. International Journal of Pharmaceutics, 2004. 286(1-2): p. 69-80.

49. Kim, E.J., et al., Preparation of a solid dispersion of felodipine using a solvent wetting method. European Journal of Pharmaceutics and Biopharmaceutics, 2006. 64(2): p. 200-5.

50. Hancock, B.C. and G. Zografi, Characteristics and significance of the amorphous state in pharmaceutical systems. Journal of Pharmaceutical Sciences, 1997. 86(1): p. 1-12.

51. Yu, L., Amorphous pharmaceutical solids: preparation, characterization and stabilization. Advanced Drug Delivery Review, 2001. 48(1): p. 27-42.

52. Abu-Diak, O.A., D.S. Jones, and G.P. Andrews, An investigation into the dissolution properties of celecoxib melt extrudates: understanding the role of polymer type and concentration in stabilizing supersaturated drug concentrations. Molecular Pharmacology, 2011. 8(4): p. 1362-71.

53. K. Sekiguchi, N.O., Studies on eutectic mixtures. I. A comparison of behavior of eutectic mixtures of sulphathiazole and that of ordinary sulphathiazole in man. Chemical and Pharmaceutical Bulletin, 1961. 9: p. 866-872.

54. Rina Chokshi, H.Z., Hot-Melt Extrusion Technique: A Review. Iranian Journal of Pharmaceutical Research, 2004. 3 p. 3-16.

55. Crowley, M.M., et al., Pharmaceutical applications of hot-melt extrusion: part I. Drug Development and Industrial Pharmacy, 2007. 33(9): p. 909-26.

56. Luxenburg, L.A., D.G. Baird, and E.G. Joseph, Background studies in the modeling of extrusion cooking processes for soy flour doughs. Biotechnology Progress, 1985. 1(1): p. 33-8.

57. Andrews, G.P., O.A. AbuDiak, and D.S. Jones, Physicochemical characterization of hot melt extruded bicalutamide-polyvinylpyrrolidone solid dispersions. Journal of Pharmaceutical Sciences, 2010. 99(3): p. 1322-35.

58. Luo, Y., et al., Improvement of dissolution and bioavailability of Ginsenosides by hot melt extrusion and cogrinding. Drug Development and Industrial Pharmacy, 2012.103: p. 1-8. 
59. Feng, J., et al., Evaluation of polymer carriers with regard to the bioavailability enhancement of bifendate solid dispersions prepared by hot-melt extrusion. Drug Development and Industrial Pharmacy, 2012. 38(6): p. 735-43.

60. Jijun, F., et al., Nimodipine (NM) tablets with high dissolution containing NM solid dispersions prepared by hot-melt extrusion. Drug Development and Industrial Pharmacy, 2011. 37(8): p. 934-44.

61. He, H., R. Yang, and X. Tang, In vitro and in vivo evaluation of fenofibrate solid dispersion prepared by hot-melt extrusion. Drug Development and Industrial Pharmacy, 2010. 36(6): p. 681-7.

62. Zheng, X., et al., Part I: characterization of solid dispersions of nimodipine prepared by hot-melt extrusion. Drug Development and Industrial Pharmacy, 2007. 33(7): p. 791-802.

63. Zheng, X., et al., Part II: bioavailability in beagle dogs of nimodipine solid dispersions prepared by hot-melt extrusion. Drug Development and Industrial Pharmacy, 2007. 33(7): p. 783-9.

64. Qi, S., et al., Characterisation of solid dispersions of paracetamol and eudragite prepared by hot-melt extrusion using thermal, microthermal and spectroscopic analysis. International Journal of Pharmaceutics, 2008. 354(1-2): p. 158-67.

65. Chokshi, R.J., et al., Characterization of physico-mechanical properties of indomethacin and polymers to assess their suitability for hot-melt extrusion processs as a means to manufacture solid dispersion/solution. Journal of Pharmaceutical Sciences, 2005. 94(11): p. 2463-74.

66. Tho, I., et al., Formation of nano/micro-dispersions with improved dissolution properties upon dispersion of ritonavir melt extrudate in aqueous media. European Journal of Pharmaceutical Sciences, 2010. 40(1): p. 25-32.

67. Ghosh, I., et al., Comparison of HPMC based polymers performance as carriers for manufacture of solid dispersions using the melt extruder. International Journal of Pharmaceutics, 2011. 419(1-2): p. 12-9.

68. Hughey, J.R., et al., Dissolution enhancement of a drug exhibiting thermal and acidic decomposition characteristics by fusion processing: a comparative study of hot melt extrusion and KinetiSol dispersing. AAPS PharmSci Technology, 2010. 11(2): p. 760-74.

69. Lakshman, J.P., et al., Application of melt extrusion in the development of a physically and chemically stable high-energy amorphous solid dispersion of a poorly water-soluble drug. Molecular Pharmacology, 2008. 5(6): p. 994-1002. 
70. Leuner, C. and J. Dressman, Improving drug solubility for oral delivery using solid dispersions. European Journal of Pharmaceutics and Biopharmceutics, 2000. 50(1): p. 47-60.

71. Cal, K. and K. Sollohub, Spray drying technique. I: Hardware and process parameters. Journal of Pharmaceutical Sciences, 2010. 99(2): p. 575-86.

72. Al-Obaidi, H., S. Brocchini, and G. Buckton, Anomalous properties of spray dried solid dispersions. Journal of Pharmaceutical Sciences, 2009. 98(12): p. 4724-37.

73. Caron, V., et al., A comparison of spray drying and milling in the production of amorphous dispersions of sulfathiazole/polyvinylpyrrolidone and sulfadimidine/polyvinylpyrrolidone. Molecular Pharmacology, 2011. 8(2): p. 53242.

74. Vehring, R., Pharmaceutical particle engineering via spray drying. Pharmaceutical Research, 2008. 25(5): p. 999-1022.

75. Zahedi, P. and P.I. Lee, Solid molecular dispersions of poorly water-soluble drugs in poly(2-hydroxyethyl methacrylate) hydrogels. European Journal of Pharmaceutics and Biopharmaceutics, 2007. 65(3): p. 320-8.

76. Wu, K., et al., Formation and characterization of solid dispersions of piroxicam and polyvinylpyrrolidone using spray drying and precipitation with compressed antisolvent. Journal of Pharmaceutical Sciences, 2009. 98(7): p. 2422-31.

77. Friesen, D.T., et al., Hydroxypropyl methylcellulose acetate succinate-based spray-dried dispersions: an overview. Molecular Pharmacology, 2008. 5(6): p. 1003-19.

78. Rodier, E., et al., A three step supercritical process to improve the dissolution rate of eflucimibe. European Journal of Pharmaceutical Sciences, 2005. 26(2): p. 18493.

79. Won, D.H., et al., Improved physicochemical characteristics of felodipine solid dispersion particles by supercritical anti-solvent precipitation process. International Journal of Pharmaceutics, 2005. 301(1-2): p. 199-208.

80. Verreck, G., et al., Preparation and characterization of nanofibers containing amorphous drug dispersions generated by electrostatic spinning. Pharmceutical Research, 2003. 20(5): p. 810-7.

81. Watanabe, T., et al., Stabilization of amorphous indomethacin by co-grinding in a ternary mixture. International Journal of Pharmaceutics, 2002. 241(1): p. 103-11. 
82. Sasfield, B.A., Powder $x$-ray diffraction detection of crystalline phases in amorphous pharmaceuticals. International Centre for Diffraction Data, 2006: p. 322-327.

83. Anthony Severdia, et al., Determination of minor quantities of polymorph in drug substance: comparison of near-infrared spectroscopy and X-ray powder diffraction. American Pharmaceutical Review, 2010. 13(2).

84. Serajuddin, A.T., Solid dispersion of poorly water-soluble drugs: early promises, subsequent problems, and recent breakthroughs. Journal of Pharmaceutical Sciences, 1999. 88(10): p. 1058-66.

85. Newman, A., et al., Characterization of amorphous API:Polymer mixtures using $X$-ray powder diffraction. Journal of Pharmaceutical Sciences, 2008. 97(11): p. 4840-56.

86. Ayenew, Z., A. Paudel, and G. Van den Mooter, Can compression induce demixing in amorphous solid dispersions? A case study of naproxen-PVP K25. European Journal of Pharmaceutics and Biopharmaceutics, 2012.

87. York., P., Solid-state properties of powders in the formulation and processing of solid dosage forms. International Journal of Pharmaceutics., 1983. 14: p. 1-28.

88. Kontny, G.Z.a.M.J., Physical characterization of pharmaceutical solids. Sorption of Water by Solids 1995, New York.

89. D.J. Burnett, F.T., and T. Sokolosk, Investigating carbamazepine-acetone solvate formation via dynamic gravimetric vapor sorption. Journal of Thermal Analysis and Calorimetry, 2007. 89: p. 693-698.

90. Mackin, L., et al., Quantification of low levels $(<10 \%)$ of amorphous content in micronised active batches using dynamic vapour sorption and isothermal microcalorimetry. International Journal of Pharmaceutics, 2002. 231(2): p. 22736.

91. Pham, T.N., et al., Analysis of Amorphous solid dispersions using 2D solid-state NMR and (1)h t(1) relaxation measurements. Molecular Pharmacology, 2010.

92. Lefort, R., et al., Solid state NMR and DSC methods for quantifying the amorphous content in solid dosage forms: an application to ball-milling of trehalose. International Journal of Pharmaceutics, 2004. 280(1-2): p. 209-19.

93. Joe, J.H., et al., Effect of the solid-dispersion method on the solubility and crystalline property of tacrolimus. International Journal of Pharmaceutics, 2010. 395(1-2): p. 161-6. 
94. Li, D.X., et al., Enhanced solubility and bioavailability of sibutramine base by solid dispersion system with aqueous medium. Biological and Pharmaceutical Bulletin, 2010. 33(2): p. 279-84.

95. B. Murúa-Pagola, C.I.B.-G., F. Martínez-Bustos Preparation of starch derivatives using reactive extrusion and evaluation of modified starches as shell materials for encapsulation of flavoring agents by spray drying. Journal of Food Engineering, 2009. 91: p. 380-386.

96. Reineccius, G., Flavor technology. In: Flavor Chemistry and Technology, 2006, FL, USA.: Taylor and Francis Group.

97. Kelly, M.R., et al., Pharmacokinetics of orally administered furosemide. Clinical Pharmacology and Therapeutics, 1974. 15(2): p. 178-86.

98. Grahnen, A., M. Hammarlund, and T. Lundqvist, Implications of intraindividual variability in bioavailability studies of furosemide. European Journal of Clinical Pharmacology, 1984. 27(5): p. 595-602.

99. Ozdemir, N. and S. Ordu, Improvement of dissolution properties of furosemide by complexation with beta-cyclodextrin. Drug Development and Industrial Pharmacy, 1998. 24(1): p. 19-25.

100. Spamer, E., et al., Characterization of the complexes of furosemide with 2hydroxypropyl-beta-cyclodextrin and sulfobutyl ether-7-beta-cyclodextrin. European Journal of Pharmaceutical Sciences, 2002. 16(4-5): p. 247-53.

101. Kata, M. and A. Antal, Enhancement of solubility of furosemide with betacyclodextrin. Pharmazie, 1984. 39(12): p. 856-7.

102. Devarakonda, B., et al., Effect of $\mathrm{pH}$ on the solubility and release of furosemide from polyamidoamine (PAMAM) dendrimer complexes. International Journal of Pharmaceutics, 2007. 345(1-2): p. 142-53.

103. Zvonar, A., et al., Microencapsulation of self-microemulsifying system: improving solubility and permeability of furosemide. International Journal of Pharmaceutics, 2010. 388(1-2): p. 151-8.

104. Salonen, J., et al., Mesoporous silicon microparticles for oral drug delivery: loading and release of five model drugs. Journal of Controlled Release, 2005. 108(2-3): p. 362-74.

105. Otsuka, M., M. Onoe, and Y. Matsuda, Hygroscopic stability and dissolution properties of spray-dried solid dispersions of furosemide with Eudragit. Journal of Pharmaceutical Sciences, 1993. 82(1): p. 32-8. 
106. Iannuccelli, V., et al., PVP solid dispersions for the controlled release of furosemide from a floating multiple-unit system. Drug Development and Industrial Pharmacy, 2000. 26(6): p. 595-603.

107. Shin, S.C. and J. Kim, Physicochemical characterization of solid dispersion of furosemide with TPGS. International Journal of Pharmaceutics, 2003. 251(1-2): p. 79-84.

108. Doherty, C. and P. York, Evidence for solid- and liquid-state interactions in a furosemide-polyvinylpyrrolidone solid dispersion. Journal of Pharmceutical Sciences, 1987. 76(9): p. 731-7.

109. Al-Obaid, A.M., et.al., Analytical profiles of drug substances. Analytical Profile of Furosemide. Vol. 18. 1989, New York: Academic press.

110. Wunderlich B., J.Y.a.B.A., Mathematical description of differential scanning calorimetry based on periodic temperature modulation. Thermochim.Acta, 1994. 238: p. 277-293.

111. Tomasi C., M.P., Hawkins N.A. and Hill V., Characterisation of amorphous materials by modulated differential scanning calorimetry. Thermochim.Acta, 1996. 278: p. 9-18.

112. Qi, S., et al., An investigation into the crystallisation behaviour of an amorphous cryomilled pharmaceutical material above and below the glass transition temperature. Journal of Pharmceutical Sciences, 2010. 99(1): p. 196-208.

113. Hancock, B.C., S.L. Shamblin, and G. Zografi, Molecular mobility of amorphous pharmaceutical solids below their glass transition temperatures. Pharmceutical Research, 1995. 12(6): p. 799-806.

114. Mark E.Davis, M.E.B., Cyclodextrin-based pharmaceutics: past, present and future. Nature Reviews, 2004. 3: p. 1023-1035.

115. Eytan A. Klausner, E.L., David Stepensky, Eva Cserepes, Miklos Barta, Michael Friedman and Amnon Hoffman, Furosemide pharmacokinetics and pharmacodynamics following gastroretentive dosage form administration to healthy volunteers. Journal of Clinical Pharmacology, 2003. 43: p. 711-720.

116. Ann Marie Kaukonen, L.L., Jarno Salonen, Jaani Tuura, and T.L. Teemu Heikkila, Jouni Hirvonen and Vesa-Pekka Lehto, Enhanced in vitro permeation of furosemide loaded into thermally carbonized mesoporous silicon (TCPSi) microparticles. European Journal of Pharmaceutics and Biopharmaceutics, 2007. 66: p. 348-356. 
117. Yoshihisa Matsuda, M.O., Mika Onoe, and Etsuko Tatsumi, Amorphism and Physicochemical Stability of Spray-dried Frusemide. Journal of Pharmacology. 1992. 44: p. 627-633.

118. Thorsteinn Loftsson, D.D., Historical Perspectives Cyclodextrins and their pharmaceutical applications. International Journal of Pharmaceutics, 2007. 329: p. 11.

119. Armin Sadighi, S.N.O., S.M. Rezayat, M. Foroutan, M.A. Faramarzi, F.A. Dorkoosh, Mathematical modelling of the transport of hydroxypropyl-bcyclodextrin inclusion complexes of ranitidine hydrochloride and furosemide loaded chitosan nanoparticles across a Caco-2 cell monolayer. International Journal of Pharmaceutics, 2012. 422: p. 479-488.

120. Wikipedia. Furosedime. Available from: http://en.wikipedia.org/wiki/Furosemide\#Precautions.2C_side_effects.2C_and_ad ministration. Accessed Feb.10 $0^{\text {th }}, 2012$.

121. Kelly MR, C.R., Forrey AW, Kimpel BM, Pharmacokinetics of orally administered furosemide Clinical Pharmacology Therapeutics, 1973. 15: p. 178186.

122. Hammarlund, M.M., Paalzow, L.K., Odlind, B., Pharamcokinetics of furosemide in man after intravenous and oral administration. Application of moment analysis. European. Journal of Clinical. Pharmacology., 1984. 26: p. 197-207.

123. G.E. Granero, M.R.L., M.J. Mora, H.E. Junginger, K.K. Midha, V.P. Shah, S. Stavchansky, and D.M. Barends, Biowaiver monographs for immediate release solid oral dosage forms: furosemide. Journal of Pharmaceutical Sciences, 2010. 99(6): p. 2544-2556.

124. Chungi VS, D.L., Smith RB, Gastrointestinal sites of furosemide absorption in rats. International Journal of Pharmaceutics, 1979. 4: p. 27-38.

125. Nicola J. Cleara, A.M., Michael Humphreya, Brian T. Henrya, Maria Wulffb, and R.J.A. Don J. Nicholsb, Ian Wilding, Evaluation of the Intelisite capsule to deliver theophylline and frusemide tablets to the small intestine and colon. European Journal of Pharmaceutical Sciences, 2001. 13: p. 375-384.

126. R. M. Amin Kreaz, G.D.a.M.K., The influence of beta-cyclodextrins on the solubility of furosemide. Journal of Inclusion Phenomena and Molecular Recognition in Chemistry, 1998. 31: p. 189-196.

127. Vlachou, M., Preparation and characterization of the inclusion complex of furosemide with hydroxypropyl- $\beta$-cyclodextrin. Journal of Biomaterial Application, 2003. 17(3): p. 197-206. 
128. R Spricigo, K.C.B., V O Consiglieri, C H Serra, Obtention and evaluation of inclusion complexes of furosemide with beta-ciclodextrin and hidroxipropil-betaciclodextrin: effects on drug's dissolution properties. Acta Farmaceutica Bonaerense, 2008. 27(5): p. 645-653.

129. Bharathi Devarakonda, D.P.O., Anja Judefeind, and M.M.d.V. Ronald A. Hill, Effect of $\mathrm{pH}$ on the solubility and release of furosemide from polyamidoamine (PAMAM) dendrimer complexes. International Journal of Pharmaceutics, 2007. 345: p. 142-153.

130. A. Zvonar, K.B., A. Kristl, M. Gasperlin, Microencapsulation of selfmicroemulsifying system: Improving solubility and permeability of furosemide. International Journal of Pharmaceutics, 2010. 388: p. 151-158.

131. J. Salonen, L.L., A.M. Kaukonen, J. Tuura, M. Bjo“ rkqvist, and K.V.h.-H. T. Heikkila, J. Hirvonen, V.-P. Lehto, Mesoporous silicon microparticles for oral drug delivery: loading and release of five model drugs. Journal of Controlled Release, 2005. 168: p. 362-374.

132. Makoto Otsuka, M.O., Yoshihisa Matsuda, Hygroscopic stability and dissolution properties of spray-dried solid dispersion of furosemide with eudragit. Journal of Pharmaceutical Sciences, 1993. 82(1): p. 33-38.

133. MK Raval, D.P., SM Varma, MA Khodifad, JM Patel, NR Sheth, Influence of some hydrophilic polymers on dissolution characteristics of furosemide through solid dispersion: An unsatisfied attempt for immediate release formulation. Journal of Pharmaceutical Negative Results, 2010. 1(2): p. 29-34.

134. Cheong-Weon Cho, J.-S.C., Sang-Chul Shin, Controlled release of furosemide from the ethylene-vinyl acetate matrix. International Journal of Pharmaceutics, 2005. 299: p. 127-133.

135. Kawano Y, I.A., Sasatsu M, Machida Y., Preparation of orally disintegrating tablets with taste-masking function: masking effect in granules prepared with correctives using the dry granulation method and evaluation of tablets prepared using the taste-masked granules. Journal of the pharmaceutical society of Japan, 2010. 130(1): p. 81-86.

136. Lingam meka, B.K., et al. Design and evaluation of polymeric coated minitablets as multiple unit gastroretentive floating drug delivery systems for furosemide. Journal of Pharmaceutical Sciences, 2009. 98(6): p. 2122-2132.

137. Michael Crothers, N.M.P.S.R., Frank Heatley, and D.A. S. Keith Nixon, Colin Booth, Solubilisation of drugs in micellar solutions of diblock copolymers of ethylene oxide and styrene oxide. International Journal of Pharmaceutics, 2008. 358 p. 303-306. 
138. Higuchi, T., Connors, K.A., Advances in Analytical Chemistry and Instrumentation. Phase solubility techniques, 1965, New York: Interscience. 


\section{VITA}

Rui Zhu was born in Xi'an, Shannxi province, China in 1978. She attended the Beijing University of Chinese Medicine in 1996 where she obtained her Bachelors of Science degree in Chinese materia medica in July of 2000. In the August of 2000, she enrolled in the Peking Union Medical College as a graduate student under the mentorship of Prof. Jianmin Chen and she obtained her Master of Science degree in July, 2003. In August 2007, she enrolled in the University of Tennessee Health Science Center (UTHSC) as a PHD graduate student under the mentorship of Dr. Johnson. During her time at UTHSC She thoroughly enjoyed the time spending here in UTHSC. Rui plans on graduating with a doctoral degree from UTHSC in May of 2012. 